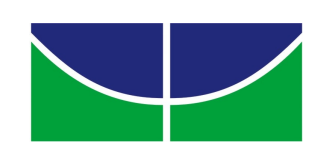

UNIVERSIDADE DE BRASÍLIA

INSTITUTO DE FÍSICA

PROGRAMA DE PÓS-GRADUAÇÃO EM FÍSICA

\title{
Comparação de métodos computacionais para o estudo da termodinâmica de sistemas com interações de longo alcance
}

\author{
Moises Fabiano Pereira da Silva Júnior
}

Dissertação de Mestrado

Brasília

Fevereiro de 2016 
Ficha catalográfica elaborada automaticamente, com os dados fornecidos pelo(a) autor(a)

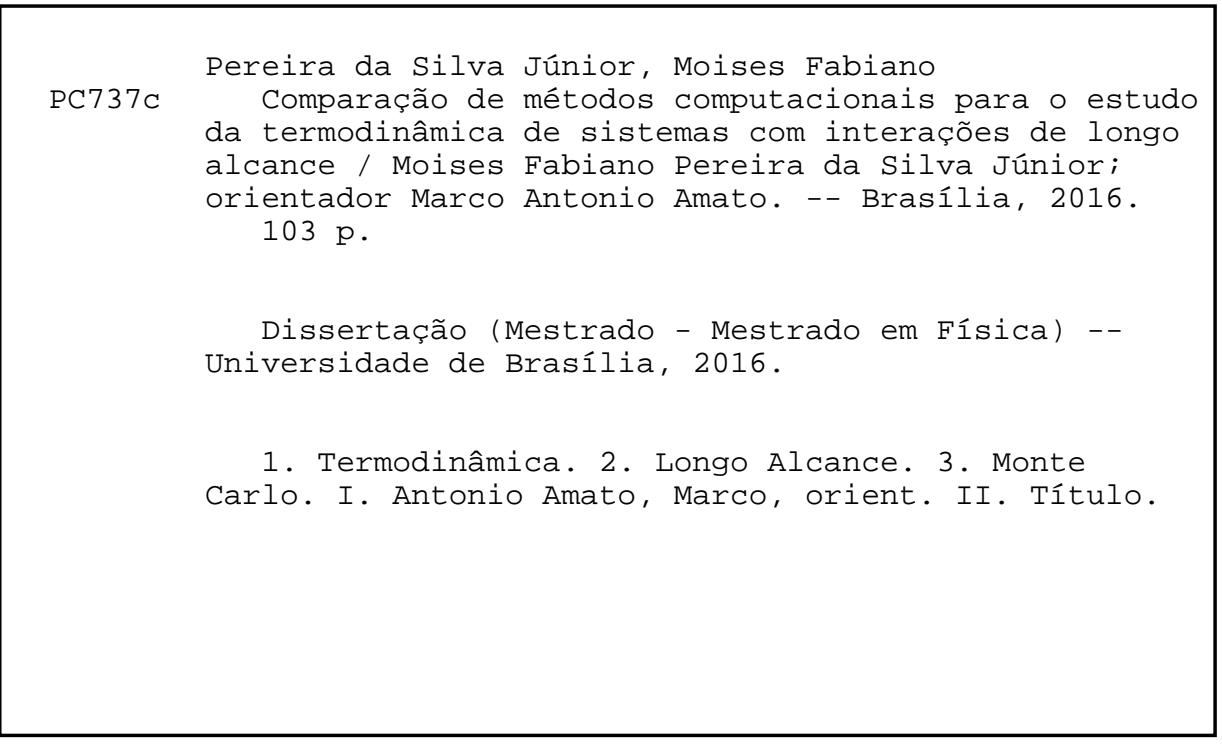


Moises Fabiano Pereira da Silva Júnior

\section{Comparação de métodos computacionais para o estudo da termodinâmica de sistemas com interações de longo alcance}

Dissertação apresentada ao Instituto de Física da Universidade de Brasília, para a obtenção de Título de Mestre em Física Teórica.

Orientador: Prof. Dr. Marco Antonio Amato

\section{Brasília}




\title{
"Comparação de métodos computacionais para o estudo da termodinâmica de sistemas com interações de longo alcance."
}

\author{
Por \\ Moisés Fabiano Pereira da Silva Jr.
}

Dissertação submetida ao Instituto de Física da Universidade de Brasília como parte dos requisitos para a obtenção do grau de Mestre em Física.

Aprovada por:

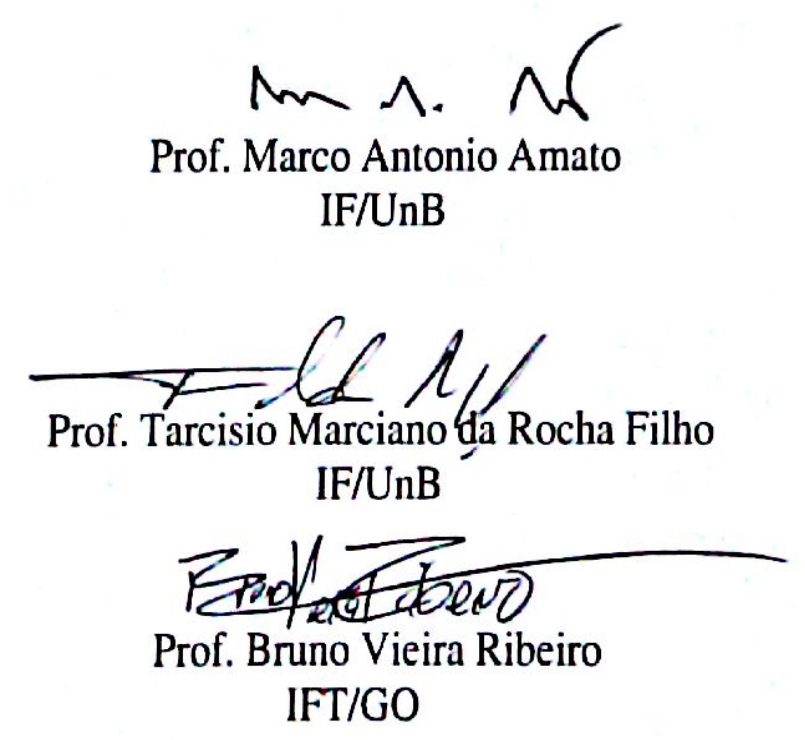

Prof. Dr. Fernando de Oliveira Albuquerque

Coordenador de Pós-Graduação

Instituto de Física 
"A pesquisa básica é como atirar uma flecha para o ar e, onde ela cair, pintar um alvo."

- Homer Adkins Burton 
Dedicado à minha máe 


\section{Agradecimentos}

Agradeço ao meu professor e orientador Marco Amato, pela confiança e paciência desde a iniciação científica. Por estar sempre disposto a dedicar seu tempo às minhas dúvidas e questionamentos.

Aos meus avós e meu pai, por terem ajudado a cuidar de mim. Em especial a minha mãe, pelo amor, carinho e por ter incentivado meus estudos desde cedo.

À Deborah, minha namorada, por estar sempre ao meu lado tornando mais fáceis os momentos difíceis e mais alegres os momentos felizes. Obrigado pelo amor, paciência e companheirismo.

Às minhas amigas Larissa e Ágatha, pelos anos de carinho, incentivo e torcida. Aos amigos da UnB Rodrigo, Arthur, Solano, Lydiane, Luciano, Daniel, Hailleen, Igor, Lilah e Waldenor, por tornarem todos os dias mais descontraídos.

A todos os meus professores, por todo o conhecimento e lições ensinadas a mim.

A todos os familiares e amigos, que de uma forma ou de outra contribuíram na minha jornada.

Ao $\mathrm{CNPq}$, pelo apoio financeiro. 


\section{Resumo}

Sistemas com interações de longe alcance podem apresentar propriedades termodinâmicas peculiares como não-aditividade, calor específico negativo e inequivalência de ensembles. Neste caso o ensemble mais conveniente para calcular as grandezas termodinâmicas é o ensemble microcanônico. Entretanto, calcular a função de partição microcanônica é, por muitas vezes, uma tarefa difícil, devido à integração sobre as posições. Por isso, métodos computacionais são importantes para resolver esse problema. Neste trabalho é feita uma comparação entre dois métodos de Monte Carlo para estudar a termodinâmica de sistemas com interação de longe alcance, com o intuito de analisar qual algoritmo é superior ao outro em diferentes situações.

Palavras-chave: Termodinâmica, Longo Alcance, Monte Carlo 


\begin{abstract}
Systems with long-range interactions may present strange thermodynamics properties as non-additivity, negative specific heat and inequivalence of ensembles. In this case the most convenient ensemble to calculate the thermodynamic quantities is the microcanonical ensemble. However, calculate the microcanonical partition function is often a hard task, due the integration over the configuration space. Thus, computational methods are important to solve these problems. In this report a comparison is made between two Monte Carlo methods to study the thermodynamics of systems with long-range interactions, in order to analyze what algorithm is superior to the other in different situations.
\end{abstract}

Keywords: Thermodynamics, Long-Range, Monte Carlo 


\section{Lista de Figuras}

1.1 Sistema composto de $\mathcal{N}$ sistemas menores. Cada sistema pode estar em qualquer um dos $W$ microestados acessíveis. . . . . . . . . . . . . . 11

1.2 Sistema em contato térmico com um reservatório de energia. . . . . . . . . 14

2.1 Partícula localizada no centro de uma esfera com distribuição homogênea de partículas. O raio da esfera exterior é $R$ e da esfera interior é $\delta$. . . . . 20

2.2 Representação de um sistema dividido em dois subsistemas que interagem entre si através de (a) interações de curto alcance e (b) interações de longo alcance. As linhas sólidas azuis representam interações entre partículas do mesmo subsistema e as linhas tracejadas vermelhas representam interações entre partículas de subsistemas diferentes. . . . . . . . . . . . . 22

2.3 Representação de um sistema de spins dividido em dois subsistemas com $\frac{N}{2}$ spins em cada um. No subsistema 1 todos os spins estão para cima e no subsistema 2 todos os spins estão para baixo. . . . . . . . . . . . . . . . 24

2.4 (a) Representação da entropia $S$ em função da energia $E$ (linha sólida) com uma região convexa e um envelope côncavo (linha tracejada). (b) Representação do inverso da temperatura $\beta$ em função da energia $E$ (linha sólida). . . . . . . . . . . . . . . . . . . 26

2.5 Fluxograma que ilustra quando temos equivalência e inequivalência entre os ensembles canônico e microcanônico. . . . . . . . . . . . . . . . . . 28

3.1 Pontos gerados por um gerador de números pseudo-aleatórios. . . . . . . . 34

4.1 (a) Entropia vs. Energia (ferromagnético). (b) Entropia vs. Energia (antiferromagnético) para o modelo de Curie-Weiss. . . . . . . . . . . . . . . . . 49

4.2 (a) Erro da entropia vs. Energia (ferromagnético). (b) Erro da entropia vs. Energia (antiferromagnético) para o modelo de Curie-Weiss. . . . . . . 50 
4.3 (a) Temperatura vs. Energia (ferromagnético). (b) Temperatura vs. Energia (antiferromagnético) para o modelo de Curie-Weiss. . . . . . . . . . . . 52

4.4 (a) Erro da temperatura vs. Energia (ferromagnético). (b) Erro da temperatura vs. Energia (antiferromagnético) para o modelo de Curie-Weiss. . . 53

4.5 Comparação de tempos de execução para diferentes critérios do método de Wang-Landau. . . . . . . . . . . . . . . . . . . . . . 54

4.6 Magnetização espontânea teórica (linha sólida) e valores obtidos numericamente através dos métodos de Ray (pontos azuis) e Wang-Landau (pontos vermelhos) para o modelo HMF-1D. . . . . . . . . . . . . . . . . .

4.7 (a) Entropia teórica (linha sólida) e valores obtidos numericamente, utilizando os métodos de Ray (pontos azuis) e Wang-Landau (pontos vermelhos), para o modelo HMF-1D. (b) Temperatura teórica (linha sólida) e valores obtidos numericamente, utilizando os métodos de Ray (pontos azuis) e Wang-Landau (pontos vermelhos), para o modelo HMF-1D. . . . . 62

4.8 Entropia configuracional do modelo HMF-1D. . . . . . . . . . . . . . . 63

4.9 Entropia do modelo HMF-1D em função da energia e magnetização. . . . . 63

4.10 (a) Entropia obtida numericamente, utilizando os métodos de Ray (pontos azuis) e Wang-Landau (pontos vermelhos), para o modelo HMF-2D. (b) Temperatura obtida numericamente, utilizando os métodos de Ray (pontos azuis) e Wang-Landau (pontos vermelhos), para o modelo HMF-2D. . . . . 65

4.11 Entropia configuracional do modelo HMF-2D. . . . . . . . . . . . . . . 66

4.12 Magnetizações e polarizações do modelo HMF-2D. . . . . . . . . . . . . . . 66

4.13 Tempos de execução dos métodos de Ray e Wang-Landau para diferentes números de particulas aplicado ao HMF-1D. . . . . . . . . . . . . . . 68

4.14 Número de passos necessários nos métodos de Ray e Wang-Landau para diferentes números de partículas aplicado ao HMF-1D.

4.15 Tempos de execução dos métodos de Ray e Wang-Landau aplicado ao HMF-

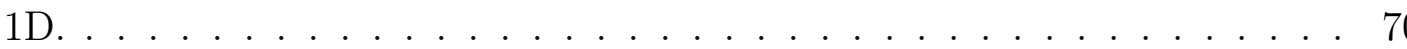

4.16 Tempos de execução entre dos métodos de Ray e Wang-Landau para diferentes números de partículas aplicado ao HMF-2D.

4.17 Número de passos necessários nos métodos de Ray e Wang-Landau para diferentes números de partículas aplicado ao HMF-2D.

4.18 Tempos de execução dos métodos de Ray e Wang-Landau aplicado ao HMF2D. 


\section{Lista de Tabelas}

4.1 Tempos médios de execução para diferentes critérios de modificação do método de Wang-Landau aplicado ao modelo de Curie-Weiss. . . . . . . . . 51

4.2 Tempos de execução com número de passos e pontos fixados para diferentes números de partículas dos métodos de Ray e Wang-Landau aplicados ao modelo HMF-1D. . . . . . . . . . . . . . . . . . . . . . . . . . . 68

4.3 Tempos de execução para diferentes números de partículas dos métodos de Ray e Wang-Landau aplicados ao modelo HMF-1D. . . . . . . . . . . . . . 69

4.4 Tempos de execução com número de passos e pontos fixados para diferentes números de partículas dos métodos de Ray e Wang-Landau aplicados ao modelo HMF-2D. . . . . . . . . . . . . . . . . . . . . . . 71

4.5 Tempos de execução para diferentes números de partículas dos métodos de Ray e Wang-Landau aplicados ao modelo HMF-2D. . . . . . . . . . . . . . 72 


\section{Lista de Símbolos}

\begin{tabular}{ll}
$E$ & Energia \\
$N$ & Número de partículas \\
$H_{N}$ & Hamiltoniana de um sistema composto de $N$ partículas \\
$\mathbf{p}$ & Momentos generalizados de $N$ partículas \\
$\mathbf{q}$ & Posições generalizadas de $N$ partículas \\
$W$ & Função de Partição Microcanônica \\
$S$ & Entropia \\
$T$ & Temperatura \\
$V$ & Volume \\
$P$ & Pressão \\
$M$ & Magnetização \\
$H$ & Campo Magnético \\
$F$ & Energia Livre de Helmholtz \\
$\mathcal{H}$ & Entalpia \\
$\kappa_{x}$ & Energia Livre de Gibbs \\
$\kappa_{x}$ & Calor Específico a $x$ constante \\
& \\
\hline &
\end{tabular}


Constante de Boltzmann

$p_{i}$

Probabilidade do sistema se encontrar no microestado $i$

$P(\mathbf{p}, \mathbf{q}) \quad$ Probabilidade do sistema se encontrar no microestado $(\mathbf{p}, \mathbf{q})$

$h \quad$ Constante de Plank

$\beta \quad$ Inverso da temperatura, $\beta=\frac{1}{k T}$

Z Função de Partição Canônica

$m$

Magnetização intensiva

$P_{+} \quad$ Polarização na direção da diagonal principal

$P_{-} \quad$ Polarização na direção da diagonal secundária

$S_{i} \quad$ Spin da partícula $i$

$K \quad$ Energia cinética

Unergia potencial

A Probabilidade de aceitação

$\omega \quad$ Densidade de probabilidade

$\mathcal{L}^{-1} \quad$ Transformada inversa de Laplace

$g \quad$ Densidade de estados estimada pelo método de Wang-Landau

$B_{\text {inv }} \quad$ Função inversa de $\frac{I_{1}}{I_{0}}$ 


\section{Sumário}

Introdução 1

1 Entropia e Ensemble Microcanônico 3

1.1 Entropia na Termodinâmica . . . . . . . . . . . . . . . 3

1.2 Potenciais Termodinâmicos . . . . . . . . . . . . . . . . 5

1.3 Entropia na Física Estatística . . . . . . . . . . . . . . . . . . . . . . . . . 9

1.4 Ensembles Estatísticos . . . . . . . . . . . . . . . . . . . . 12

2 Sistemas com Interações de Longo Alcance 19

2.1 Definição . . . . . . . . . . . . . . . . . . . . . . 19

2.2 Aditividade e Não-Aditividade . . . . . . . . . . . . . . . . . . . . . 21

2.3 Equivalência e Inequivalência de Ensemble . . . . . . . . . . . . . . . . . 24

2.4 Exemplos . . . . . . . . . . . . . . . . . . . . . . . . . 28

3 Método de Monte Carlo Microcanônico 33

3.1 Visão Geral . . . . . . . . . . . . . . . . . . . . . . . . . 33

3.2 Método de Metropolis . . . . . . . . . . . . . . . . . . . 35

3.3 Método de Ray . . . . . . . . . . . . . . . . . . . . 37

3.4 Método de Wang-Landau . . . . . . . . . . . . . . . . . . . . 40

4 Resultados $\quad 46$

4.1 Curie-Weiss . . . . . . . . . . . . . . . . . . . . 46

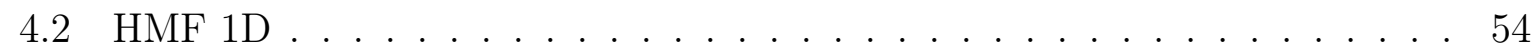

$4.3 \mathrm{HMF} 2 \mathrm{D} \ldots \ldots \ldots \ldots \ldots \ldots \ldots$

4.4 Discussões . . . . . . . . . . . . . . . . . . . . 67

$\begin{array}{ll}\text { Conclusão } & 74\end{array}$

$\begin{array}{ll}\text { Apêndice A Códigos Fonte } & 75\end{array}$

Referências Bibliográficas $\quad 85$ 


\section{Introdução}

Sistemas com interações de longo alcance surgem em diversas áreas da física. Podemos encontrar esses tipos de sistemas em astrofísica, física de plasma, hidrodinâmica, física atômica e física nuclear. Uma interação é dita ser de longo alcance se o potencial decai, a longas distâncias, como $r^{-\alpha} \operatorname{com} \alpha \leq d$, onde $d$ é a dimensão espacial, $r$ é a distância entre duas partículas e $\alpha$ uma constante, [6]. A característica principal de um sistema com interação de longo alcance é a não-aditividade. Por exemplo: dado um sistema dividido em duas partes, A e B, que interagem entre si, a energia total do sistema é

$$
E_{\text {total }}=E_{A}+E_{B}+E_{A B}
$$

com $E_{A}, E_{B}$ e $E_{A B}$ sendo a energia da parte $\mathrm{A}$, a energia da parte $\mathrm{B}$ e a energia de interação entre A e B, respectivamente. Se o sistema possui uma interação de curto alcance, então $E_{A B} \ll E_{A}+E_{B}$, e podemos desprezar a energia de interação, ficando com $E_{\text {total }} \simeq E_{A}+E_{B}$. Como a energia total é a soma das energias de cada parte, dizemos que o sistema é aditivo. Caso o sistema possua uma interação de longo alcance, não podemos desprezar a energia de interação e, com isso, a energia total do sistema não é dada pela soma das energias de cada parte, $E_{\text {total }} \neq E_{A}+E_{B}$, logo, temos um sistema não-aditivo.

Outra característica recorrente em sistemas com interações de longo alcance é o calor específico negativo, que leva a inequivalência entre os ensembles microcanônico e canônico no cálculo de grandezas termodinâmicas. Essa inequivalência faz com que a abordagem microcanônica seja mais adequada para estudar esses tipos de sistemas. A função de partição microcanônica, em função da energia $E$ e do volume $V$, é

$$
W_{N}(E, V)=C(N) \int \delta\left(E-H_{N}(\mathbf{p}, \mathbf{q})\right) d \mathbf{p} d \mathbf{q}
$$

com $H_{N}(\mathbf{p}, \mathbf{q})$ sendo a hamiltoniana de um sistema composto de $N$ partículas e $C(N)$ uma função de $N$. Calcular a função de partição microcanônica é uma terafa árdua e, 
muitas vezes, impossível de se realizar analiticamente. Por esse motivo, é necessário o uso de métodos computacionais para estudar a termodinâmica de tais sistemas.

Em 1991, John R. Ray, seguindo a mesma ideia do trabalho de Metropolis et al., desenvolveu um método de Monte Carlo Microcanônico, baseado no trabalho de Pearson et al. Em 2001, F. Wang e D. P. Landau desenvolveram um método de Monte Carlo para calcular densidade de estados de sistemas discretos através de um passeio aleatório na energia. Mais tarde, o método foi generalizado para sistemas contínuos por Shell et al. O objetivo deste trabalho é comparar os dois métodos, a fim de saber qual apresenta melhores resultados em diferentes situações.

Este trabalho está organizado da seguinte maneira. No Capítulo 1 é feita uma revisão sobre os potenciais termodinâmicos, em especial a entropia, e da construção dos ensembles estatísticos. No Capítulo 2 é realizada uma análise mais detalhada de sistemas com interações de longo alcance, da origem da inequilavência entre ensembles e são apresentados exemplos de sistemas desse tipo. No Capítulo 3 são expostos com detalhes os métodos de Monte Carlo citados acima. Por fim, no Capítulo 4 são apresentados os resultados e discussões. 


\section{Capítulo 1}

\section{Entropia e Ensemble Microcanônico}

A entropia é o potencial termodinâmico diretamento ligado ao ensemble microcanônico. Neste capítulo, vemos o surgimento do conceito de entropia e sua relação com outros potenciais termodinâmicos. Em seguida vemos uma interpretação estatística para a entropia e a construção dos ensembles a partir dela.

\subsection{Entropia na Termodinâmica}

A essência da termodinâmica é descrever um sistema físico, composto de vários constituintes, através de poucos parâmetros macroscópicos, [11]. Encontrar as equações de movimento de $10^{23}$ partículas é uma tarefa difícil, senão impossível. Por conta disso, ao invés de descrever um sistema através da dinâmica das $10^{23}$ partículas que o compõe, podemos usar o equilíbrio termodinâmico para reduzir o número de variáveis necessárias para descrevê-lo. Ou seja, deve existir uma função, que depende apenas de variáveis macroscópicas, capaz de descrever por completo o comportamento termodinâmico de um sistema. Essa função é a entropia, pela segunda lei da termodinâmica, [10]:

Um macroestado de um sistema pode ser caracterizado por uma quantidade S, denominada entropia. Em qualquer processo, para o sistema ir de um macroestado A para um macroestado $B$, se isolado, a entropia tende a crescer, ou seja:

$$
\Delta S \geq 0
$$

A segunda lei define o equilíbrio termodinâmico de um sistema como sendo o macroestado que possui maior entropia. Algumas coisas merecem ser destacadas da segunda lei da 
termodinâmica: a primeira é que ela é válida para sistemas isolados, ou seja, se vários sistemas interagem entre si e alteram seus estados termodinâmicos, então o que não pode decrescer é a entropia total, que é a soma das entropias de todos os sistemas que participam do processo. Porém, o mais importante é que a entropia depende do macroestado do sistema, ou seja, ela não é uma função do estado microscópico (microestado) do sistema. Sistemas idênticos em diferentes microestados podem ter a mesma entropia, se estiverem no mesmo macroestado, e sistemas no mesmo microestado podem ter entropias diferentes, se estiverem em diferentes macroestados.

Um sistema isolado, composto de $N$ partículas, tem como variáveis macroscópicas grandezas extensivas (proporcionais ao tamanho do sistema) como energia interna $E$, volume $V$ (sistemas tipo fluido), magnetização $M$ (sistemas magnéticos). Ou seja, $S=S_{N}(E, V)$, para sistemas tipo fluido, e $S=S_{N}(E, M)$, para sistemas magnéticos. Toda a informação sobre o sistema está contida na entropia, porém muitas vezes estamos interessados em processos associados à mudanças nos parâmetros extensivos, então devemos tomar a forma diferencial de $S$, [5]. Definindo:

(a) $\left(\frac{\partial S}{\partial E}\right)_{V} \equiv \frac{1}{T},(b)\left(\frac{\partial S}{\partial V}\right)_{E} \equiv \frac{P}{T},(c)\left(\frac{\partial S}{\partial E}\right)_{M} \equiv \frac{1}{T},(d)\left(\frac{\partial S}{\partial M}\right)_{E} \equiv-\frac{H}{T}$.

com $T, P$ e $H$ sendo a temperatura, a pressão e o campo magnético, respectivamente. Essas variáveis são intensivas (independentes do tamanho do sistema) e, definidas como em (1.2), estão de acordo com o conceito físico de temperatura, pressão e campo magnético, $[5,23,26]$. Com isso, os diferenciais da entropia, para sistemas tipo fluido e magnéticos, são dados por

$$
\begin{gathered}
d S=\frac{1}{T} d E+\frac{P}{T} d V, \\
d S=\frac{1}{T} d E-\frac{H}{T} d M,
\end{gathered}
$$

Pela segunda lei da termodinâmica, no equilíbrio, a entropia de um sistema isolado deve ser máxima, ou seja, ela deve satisfazer $d S=0$ e $d^{2} S<0$, [5]. Considere dois subsistemas idênticos e aditivos, cada um com energia $E$ e entropia $S=S(E)$. A entropia total é $S_{\text {total }}=2 S(E)$. Se um dos subsistemas receber uma quantidade de energia $\Delta E$ do outro subsistema, então a nova entropia total será $S_{\text {total }}=S(E+\Delta E)+S(E-\Delta E)$. 
Como inicialmente os subsistemas estavam em equilíbrio térmico, de (1.2), fica claro que devemos ter

$$
S(E+\Delta E)+S(E-\Delta E) \leq 2 S(E)
$$

No limite em que $\Delta E \rightarrow 0$, temos a forma diferencial

$$
\frac{\partial^{2} S}{\partial E^{2}} \leq 0
$$

Entretanto a condição (1.6) só vale para $\Delta E \rightarrow 0$ enquanto a condição de concavidade (1.5) vale para todo $\Delta E,[5]$. O mesmo argumento pode ser feito em relação as variáveis $V$ e $M$ levando às expressões

$$
\text { (a) } \frac{\partial^{2} S}{\partial V^{2}} \leq 0,(b) \frac{\partial^{2} S}{\partial M^{2}} \leq 0
$$

\subsection{Potenciais Termodinâmicos}

Na seção 1.1 temos o surgimento da entropia com a segunda lei da termodinâmica, porém a entropia não é o único potencial termodinâmico de onde podemos extrair informações sobre nosso sistema. Outro exemplo é a energia interna, que possui uma relação direta com a entropia. Por simples inversão de variáveis temos $E=E_{N}(S, V)$, para sistemas tipo fluido, e $E=E_{N}(S, M)$, para sistemas magnéticos. Além da entropia $S$ e da energia interna $E$, outros exemplos de potenciais termodinâmicos são a energia livre de Helmholtz $F$, a entalpia $\mathcal{H}$ e a energia livre de Gibbs $G$. Os potenciais termodinâmicos podem ser funções de grandezas extensivas ou de grandezas intensivas. De (1.3) e (1.4), temos que as variações infinitesimais da energia interna, de sistemas tipo fluido e magnéticos, são dadas por:

$$
\begin{gathered}
d E=T d S-P d V . \\
d E=T d S+H d M .
\end{gathered}
$$

Das equações (1.8)-(1.4), temos que: 


$$
\text { (a) }\left(\frac{\partial E}{\partial S}\right)_{V}=T,(b)\left(\frac{\partial E}{\partial V}\right)_{S}=-P,(c)\left(\frac{\partial E}{\partial S}\right)_{M}=T,(d)\left(\frac{\partial E}{\partial M}\right)_{S}=H
$$

No equilíbrio, quando a entropia é máxima a energia interna é mínima, [5]. Então a condição de concavidade para a entropia é uma condição de convexidade para a energia, com isso

$$
\text { (a) } \frac{\partial^{2} E}{\partial S^{2}} \geq 0,(b) \frac{\partial^{2} E}{\partial V^{2}} \geq 0,(c) \frac{\partial^{2} E}{\partial M^{2}} \geq 0 .
$$

A energia interna se relaciona com os outros potenciais termodinâmicos através de transformadas de Legendre, [5],

$$
\begin{gathered}
F=E-T S, \\
\mathcal{H}=E+P V \text { ou } \mathcal{H}=E-H M, \\
G=E-T S+P V \text { ou } G=E-T S-H M,
\end{gathered}
$$

Fazendo o diferencial das equações (1.12)-(1.14) e usando (1.8) e (1.9):

$$
\begin{gathered}
\text { (a) } d F=-S d T-P d V, \text { (b) } d F=-S d T+H d M, \\
\text { (a) } d \mathcal{H}=T d S+V d P, \text { (b) } d \mathcal{H}=T d S-M d H, \\
\text { (a) } d G=-S d T+V d P, \text { (b) } d G=-S d T-M d H .
\end{gathered}
$$

Das equações (1.15)-(1.17), podemos concluir que, nos sistemas tipo fluido $F=F_{N}(T, V), \mathcal{H}=$ $\mathcal{H}_{N}(S, P), G=G_{N}(T, P)$ e nos sistemas magnéticos $F=F_{N}(T, M), \mathcal{H}=\mathcal{H}_{N}(S, H), G=$ $G_{N}(T, H)$. Portanto, das equações (1.15)-(1.17), temos que:

(a) $\left(\frac{\partial F}{\partial T}\right)_{V}=-S,(b)\left(\frac{\partial F}{\partial V}\right)_{T}=-P,(c)\left(\frac{\partial F}{\partial T}\right)_{M}=-S,(d)\left(\frac{\partial F}{\partial M}\right)_{T}=H$

(a) $\left(\frac{\partial \mathcal{H}}{\partial S}\right)_{P}=T,(b)\left(\frac{\partial \mathcal{H}}{\partial P}\right)_{S}=V,(c)\left(\frac{\partial \mathcal{H}}{\partial S}\right)_{H}=T,(d)\left(\frac{\partial \mathcal{H}}{\partial H}\right)_{S}=-M$ 
(a) $\left(\frac{\partial G}{\partial T}\right)_{P}=-S,(b)\left(\frac{\partial G}{\partial P}\right)_{T}=V,(c)\left(\frac{\partial G}{\partial T}\right)_{H}=-S,(d)\left(\frac{\partial G}{\partial H}\right)_{T}=-M$

As equações (1.2), (1.10) e (1.18)-(1.20) são chamadas equações de estado. Equações de estado são relações entre as variáveis do estado de um sistema termodinâmico, onde a partir delas temos o comportamento do sistema. Uma das propriedades da transformada de Legendre é que, se uma função $Y(X)$ é convexa em $X$, então sua transformada $\Psi(\Phi)$ será côncava em $\Phi$. Com isso, de (1.12)-(1.14)

$$
\begin{aligned}
& \text { (a) } \frac{\partial^{2} F}{\partial T^{2}} \leq 0, \quad(b) \frac{\partial^{2} F}{\partial V^{2}} \geq 0, \quad(c) \frac{\partial^{2} F}{\partial M^{2}} \geq 0 . \\
& \text { (a) } \frac{\partial^{2} \mathcal{H}}{\partial S^{2}} \geq 0, \quad(b) \frac{\partial^{2} \mathcal{H}}{\partial P^{2}} \leq 0, \quad(c) \frac{\partial^{2} \mathcal{H}}{\partial H^{2}} \leq 0 . \\
& \text { (a) } \frac{\partial^{2} G}{\partial T^{2}} \leq 0, \quad \text { (b) } \frac{\partial^{2} G}{\partial P^{2}} \leq 0, \quad(c) \frac{\partial^{2} G}{\partial H^{2}} \leq 0 .
\end{aligned}
$$

As derivadas de segunda ordem dos potenciais termodinâmicos geralmente descrevem a resposta do sistemas a pequenas mudanças. Exemplos são o calor específico, que mede a absorção de calor por uma variação na temperatura, a compressibilidade, que mede a resposta do volume a uma variação da pressão, e a susceptibilidade, que mede a capacidade de um material de magnetizar-se sob a ação de um campo magnético, [23]. Usando as equações (1.2), (1.10) e (1.18)-(1.20):

(i) Calor específico a $x$ constante: $C_{x} \equiv T\left(\frac{\partial S}{\partial T}\right)_{x}$.

- Volume constante:

$$
C_{V}=T\left(\frac{\partial S}{\partial T}\right)_{V}=\left(\frac{\partial E}{\partial T}\right)_{V}=-T\left(\frac{\partial^{2} F}{\partial T^{2}}\right)_{V} .
$$

- Pressão constante:

$$
C_{P}=T\left(\frac{\partial S}{\partial T}\right)_{P}=\left(\frac{\partial \mathcal{H}}{\partial T}\right)_{P}=-T\left(\frac{\partial^{2} G}{\partial T^{2}}\right)_{P} .
$$

- Magnetização constante: 


$$
C_{M}=T\left(\frac{\partial S}{\partial T}\right)_{M}=\left(\frac{\partial E}{\partial T}\right)_{M}=-T\left(\frac{\partial^{2} F}{\partial T^{2}}\right)_{M} .
$$

- Campo magnético constante:

$$
C_{H}=T\left(\frac{\partial S}{\partial T}\right)_{H}=\left(\frac{\partial \mathcal{H}}{\partial T}\right)_{H}=-T\left(\frac{\partial^{2} G}{\partial T^{2}}\right)_{H}
$$

(ii) Compressibilidade a $x$ constante: $\kappa_{x} \equiv-\frac{1}{V}\left(\frac{\partial V}{\partial P}\right)_{x}$.

- Compressibilidade isotérmica:

$$
\kappa_{T}=-\frac{1}{V}\left(\frac{\partial V}{\partial P}\right)_{T}=-\frac{1}{V}\left(\frac{\partial^{2} G}{\partial P^{2}}\right)_{T} .
$$

- Compressibilidade adiabática:

$$
\kappa_{S}=-\frac{1}{V}\left(\frac{\partial V}{\partial P}\right)_{S}=-\frac{1}{V}\left(\frac{\partial^{2} \mathcal{H}}{\partial P^{2}}\right)_{S} .
$$

(iii) Susceptibilidade a $x$ constante: $\chi_{x} \equiv\left(\frac{\partial M}{\partial H}\right)_{x}$.

- Susceptibilidade isotérmica:

$$
\chi_{T}=\left(\frac{\partial M}{\partial H}\right)_{T}=-\left(\frac{\partial^{2} G}{\partial H^{2}}\right)_{T} .
$$

- Susceptibilidade adiabática:

$$
\chi_{S}=\left(\frac{\partial M}{\partial H}\right)_{S}=-\left(\frac{\partial^{2} \mathcal{H}}{\partial H^{2}}\right)_{S} .
$$

Existem outros potenciais termodinâmicos, que não foram apresentados aqui, a partir dos quais pode-se extrair outras informações de um sistema físico. Isso mostra a importância de conhecer os potenciais para o estudo da termodinâmica. Porém, é necessária uma forma de obter os potenciais termodinâmicos, dado um sistema qualquer. Para isso, é preciso entender qual o significado físico deles. 


\subsection{Entropia na Física Estatística}

A entropia é o potencial termodinâmico que possui uma interpretação estatística, graças a segunda lei da termodinâmica. O macroestado de equilibro de um sistema, estado de maior entropia, pode ser visto como o estado mais provável para um dado conjunto de variáveis macroscópicas fixas, como energia $E$, volume $V$ e número de constituintes $N$, uma vez que a segunda lei da termodinâmica é válida para sistemas isolados. Como, em um sistema isolado, todos os microestados acessíveis são equiprováveis, o macroestado mais provável é aquele que possui o maior número de microestados $W$. Então, deve haver uma relação entre a entropia e o número de microestados, [16]:

$$
S=f(W)
$$

onde $f(W)$ é uma função qualquer de $W$. A entropia possui a propriedade de ser aditiva, ou seja, dois sistemas, $A$ e $B$, com entropias $S_{A}$ e $S_{B}$, possuem uma entropia total

$$
S_{\text {total }}=S_{A}+S_{B}
$$

No entanto, o número de microestatos total é dado por

$$
W_{\text {total }}=W_{A} W_{B}
$$

com $W_{A}$ e $W_{B}$ sendo o número de microestados dos sistemas $A$ e $B$, respectivamente. Uma vez que $S$ é aditiva e $W$ é multiplicativo, a função $f(W)$ deve satisfazer a seguinte condição:

$$
f\left(W_{A} W_{B}\right)=f\left(W_{A}\right)+f\left(W_{B}\right) .
$$

Diferenciando ambos os lados de (1.35) em relação a $W_{A}$, temos

$$
W_{B} f^{\prime}\left(W_{A} W_{B}\right)=f^{\prime}\left(W_{A}\right)
$$

e diferenciando ambos os lados de (1.35) em relação a $W_{B}$, temos

$$
W_{A} f^{\prime}\left(W_{A} W_{B}\right)=f^{\prime}\left(W_{B}\right)
$$

onde as linhas em (1.36) e (1.37) representam derivadas em relação ao argumento da 
função. Das equações (1.36) e (1.37):

$$
W_{A} f^{\prime}\left(W_{A}\right)=W_{B} f^{\prime}\left(W_{B}\right)
$$

Como o lado esquerdo da equação (1.38) não depende de $W_{B}$ e o lado direito não depende de $W_{A}$, ambos devem ser iguais a uma constante $k$, ou seja,

$$
W f^{\prime}(W)=k \Rightarrow f(W)=k \ln W+\text { constante }
$$

substituindo (1.39) em (1.35), tem-se que o valor da constante de integração é igual a zero. Substituindo (1.39) em (1.32), temos que a entropia de um sistema isolado é dada por:

$$
S=k \ln W
$$

A expressão (1.40) foi originalmente formulada por Ludwig Boltzmann, em 1872, mas só foi escrita da presente forma por Plank, em 1900, com $k=1,38 \times 10^{-23} \mathrm{~J} / \mathrm{K}$ sendo a constante de Boltzmann. A entropia de Boltzmann é válida para sistemas isolados, onde os microestados acessíveis do sistema são equiprováveis. A generalização para quando os microestados não são, necessariamente, equiprováveis, veio com J. Willard Gibbs, em 1878:

$$
S=-k \sum_{i=1}^{W} p_{i} \ln p_{i}
$$

$\operatorname{com} p_{i}$ sendo a probabilidade do sistema estar no microestado $i$. Uma expressão semelhante a (1.41) também foi encontrada mais tarde por Claude E. Shannon [21], em 1949, no estudo sobre teoria da informação. Uma demonstração simples de como a expressão (1.41) surge, no contexto da teoria da informação, pode ser encontrada na referência [12]. Uma maneira de se chegar a expressão (1.41) é imaginar um sistema composto de $\mathcal{N}$ sistemas menores, idênticos uns aos outros, Figura 1.1. Os sistemas podem estar em qualquer um dos $W$ microestados acessíveis. O sistema composto está isolado, portanto sua entropia é dada por

$$
\mathcal{S}=k \ln \mathcal{W},
$$




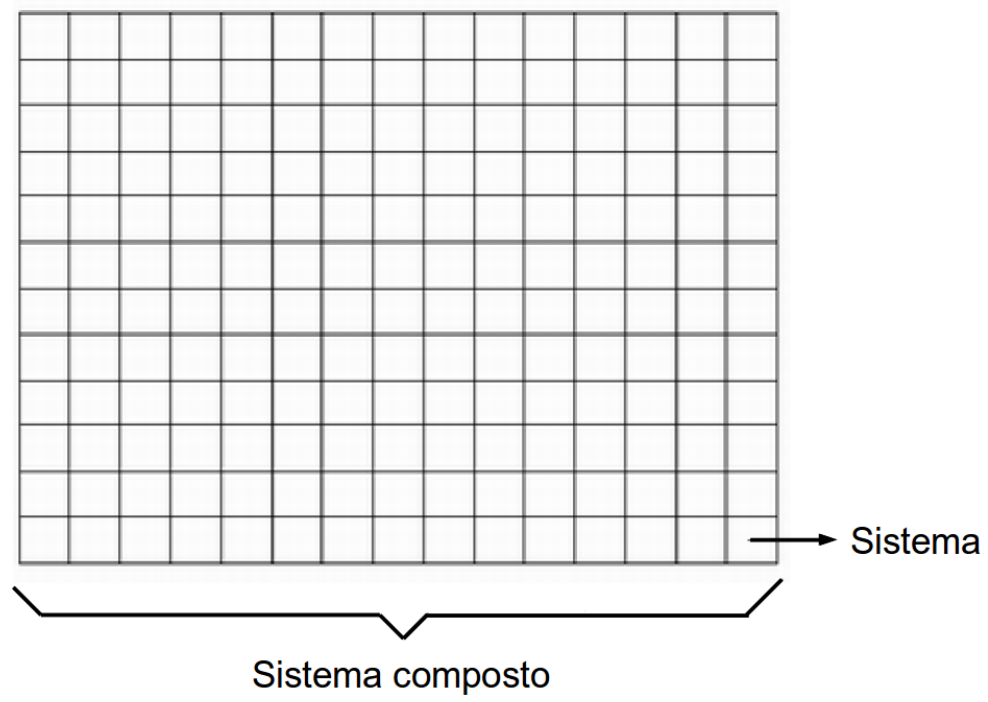

Figura 1.1: Sistema composto de $\mathcal{N}$ sistemas menores. Cada sistema pode estar em qualquer um dos $W$ microestados acessíveis.

com $\mathcal{S}$ e $\mathcal{W}$ sendo a entropia e o número de microestados do sistema composto, respectivamente. $\mathrm{O}$ número de microestados $\mathcal{W}$ do sistema composto, é obtido a partir de

$$
\mathcal{W}=\sum_{\left\{n_{i}\right\}} \frac{\mathcal{N} !}{\prod_{i=1}^{W} n_{i} !},
$$

com $n_{i}$ sendo o número de sistemas no microestado $i$. O somatório é realizado sobre todos os $\left\{n_{i}\right\}$ compatíveis com o macroestado do sistema composto. Devido ao comportamento da função fatorial, podemos aproximar o valor do somatório em (1.43) pelo maior termo da soma,

$$
\mathcal{W} \simeq \frac{\mathcal{N} !}{\prod_{i=1}^{W} n_{i}^{*} !},
$$

onde $\left\{n_{i}^{*}\right\}$ é o conjunto que maximiza $\frac{\mathcal{N} !}{\prod_{i=1}^{W} n_{i} !}$. A probabilidade de encontrar um sistema no microestado $i$ é $p_{i}=\frac{n_{i}^{*}}{\mathcal{N}}$, com

$$
\sum_{i=1}^{W} n_{i}^{*}=\mathcal{N} \rightarrow \sum_{i=1}^{W} p_{i}=1 .
$$

Aplicando o logaritmo em (1.44) e utilizando a aproximação de Stirling, $\ln x ! \simeq x \ln x-x$, temos que 


$$
\begin{aligned}
\ln \mathcal{W}= & \ln \mathcal{N} !-\sum_{i=1}^{W} \ln n_{i}^{*} ! \simeq \\
& =\mathcal{N} \ln \mathcal{N}-\mathcal{N}-\sum_{i=1}^{W} n_{i}^{*} \ln n_{i}^{*}+\sum_{i=1}^{W} n_{i}^{*}= \\
& =\mathcal{N} \ln \mathcal{N}-\sum_{i=1}^{W} n_{i}^{*} \ln n_{i}^{*}= \\
& =\mathcal{N} \sum_{i=1}^{W} p_{i} \ln \mathcal{N}-\mathcal{N} \sum_{i=1}^{W} \frac{n_{i}^{*}}{\mathcal{N}} \ln n_{i}^{*}= \\
& =\mathcal{N} \sum_{i=1}^{W} p_{i} \ln \mathcal{N}-\mathcal{N} \sum_{i=1}^{W} p_{i} \ln n_{i}^{*}= \\
= & -\mathcal{N} \sum_{i=1}^{W} p_{i}\left[\ln n_{i}^{*}-\ln \mathcal{N}\right]=-\mathcal{N} \sum_{i=1}^{W} p_{i} \ln \frac{n_{i}^{*}}{\mathcal{N}}, \\
& \therefore \ln \mathcal{W}=-\mathcal{N} \sum_{i=1}^{W} p_{i} \ln p_{i} .
\end{aligned}
$$

Substituindo (1.46) em (1.42), temos a entropia do sistema composto é dada por

$$
\mathcal{S}=-\mathcal{N} k \sum_{i=1}^{W} p_{i} \ln p_{i} .
$$

Portanto, a entropia de um sistema qualquer é dada por

$$
S=\frac{\mathcal{S}}{\mathcal{N}}=-k \sum_{i=1}^{W} p_{i} \ln p_{i},
$$

que é a mesma expressão de (1.41).

\subsection{Ensembles Estatísticos}

Uma vez obtidas as probabilidades de um sistema se encontrar em qualquer microestado $i$, temos a entropia do sistema em questão, pela expressão (1.41). Resta agora obter os $p_{i}^{\prime} s$ para diferentes situações. Aqui é apresentado para duas situações, o caso de um sistema isolado (ensemble microcanônico) e o caso de um sistema em contato térmico com um reservatório de energia (ensemble canônico). Outros ensembles seguem as mesmas ideias, mudando apenas a natureza do reservatório com que o sistema interage. 
- Ensemble Microcanônico

O ensemble microcanônico corresponde a um sistema isolado. Neste caso, todos os microestados acessíveis ao sistema são equiprováveis, $p_{i}=p, \forall i$. Temos que,

$$
\sum_{i=1}^{W} p_{i}=\sum_{i=1}^{W} p=p W=1 \rightarrow p=\frac{1}{W} .
$$

Substituindo (1.49) em (1.41), temos que a entropia microcanônica é dada por

$$
\begin{gathered}
S=-k \sum_{i=1}^{W} p_{i} \ln p_{i}=-k \sum_{i=1}^{W} p \ln p= \\
=-k \sum_{i=1}^{W} \frac{1}{W} \ln \frac{1}{W}=-k \frac{W}{W} \ln \frac{1}{W}=-k \ln \frac{1}{W}, \\
\therefore S=k \ln W .
\end{gathered}
$$

A expressão (1.50) é a entropia de Boltzmann (1.40), como era de se esperar. No caso de um sistema isolado, temos energia $E$, volume $V$ e número de constituintes do sistema $N$ conservados, ou seja, $S=S_{N}(E, V)$. No caso de sistemas discretos, $W$ é obtido por métodos de contagem, quando o sistema é contínuo, o número de microestados do sistema é obtido a partir de, [11],

$$
W_{N}(E, V)=\frac{\epsilon_{0}}{h^{d N} N !} \int \delta\left(E-H_{N}(\mathbf{p}, \mathbf{q})\right) d \mathbf{p} d \mathbf{q}
$$

com $H_{N}(\mathbf{p}, \mathbf{q})$ sendo a hamiltoniana de um sistema composto de $N$ partículas, $h$ a constante de Plank, $\epsilon_{0}$ uma constante qualquer com unidade de energia e $d$ a dimensão do sistema. A expressão (1.51) é chamada Função de Partição Microcanônica.

\section{- Ensemble Canônico}

O ensemble canônico corresponde a um sistema em contato térmico com um reservatório de energia, Figura 1.2. Seja $E_{i}$ a energia do sistema e $\tilde{E}_{r}$ a energia do reservatório, correspondente a um microestado $i$ do sistema, a energia total é dada pela soma da energia do reservatório e a energia do sistema, no caso em que a energia é aditiva, 


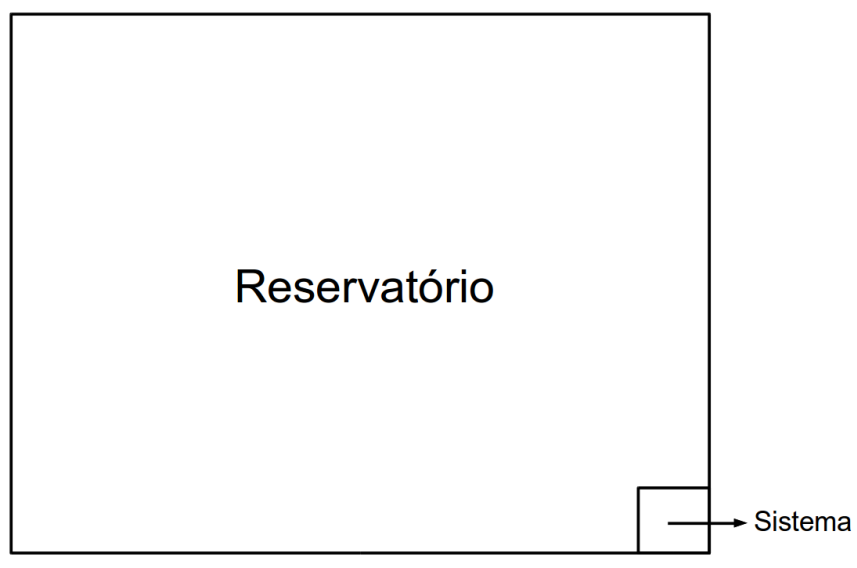

Figura 1.2: Sistema em contato térmico com um reservatório de energia.

$$
E_{0}=\tilde{E}_{r}+E_{i}
$$

com $E_{0}$ sendo a energia total, que é conservada. A probabilidade do sistema estar no microestado $i$, com energia $E_{i}$, é proporcional ao número de microestados do reservatório com energia $\tilde{E}_{r}$,

$$
p_{i} \propto \tilde{W}\left(\tilde{E}_{r}\right)
$$

sendo $\tilde{W}$ o número de microestados do reservatório. Como o reservatório é muito maior do que o sistema espera-se que $\tilde{E}_{r} \gg E_{i}$, no equilíbro. Com isso, podemos expandir $\ln \tilde{W}\left(\tilde{E}_{r}\right)$ em torno de $E_{0}$.

$$
\begin{aligned}
\ln \tilde{W}\left(\tilde{E}_{r}\right) & =\ln \tilde{W}\left(E_{0}\right)+\left(\frac{\partial \ln \tilde{W}\left(\tilde{E}_{r}\right)}{\partial \tilde{E}_{r}}\right)_{\tilde{E}_{r}=E_{0}}\left(\tilde{E}_{r}-E_{0}\right) \\
& +\frac{1}{2 !}\left(\frac{\partial^{2} \ln \tilde{W}\left(\tilde{E}_{r}\right)}{\partial \tilde{E}_{r}^{2}}\right)_{\tilde{E}_{r}=E_{0}}\left(\tilde{E}_{r}-E_{0}\right)^{2}+\cdots
\end{aligned}
$$

Usando (1.52) e mantendo apenas os termos de primeira ordem em $E_{i}$

$$
\begin{array}{r}
\ln \tilde{W}\left(\tilde{E}_{r}\right) \simeq \ln \tilde{W}\left(E_{0}\right)-\beta E_{i}, \\
\operatorname{com} \beta \equiv\left(\frac{\partial \ln \tilde{W}\left(\tilde{E}_{r}\right)}{\partial \tilde{E}_{r}}\right)_{\tilde{E}_{i}=E_{0}} . \operatorname{De}(1.53) \text { e }(1.54) \\
p_{i}=\alpha \tilde{W}\left(E_{0}\right) e^{-\beta E_{i}}
\end{array}
$$


com $\alpha$ sendo uma constante. Usando (1.45), ficamos com

$$
\begin{gathered}
\sum_{i=1}^{W} p_{i}=\sum_{i=1}^{W} \alpha \tilde{W}\left(E_{0}\right) e^{-\beta E_{i}} \\
=\alpha \tilde{W}\left(E_{0}\right) \sum_{i=1}^{W} e^{-\beta E_{i}}=1 \\
\therefore \alpha \tilde{W}\left(E_{0}\right)=\frac{1}{\sum_{i=1}^{W} e^{-\beta E_{i}}} .
\end{gathered}
$$

Substituindo (1.56) em (1.55), temos

$$
p_{i}=\frac{e^{-\beta E_{i}}}{Z}
$$

com $Z$ sendo a Função de Partição Canônica, dada por

$$
Z=\sum_{i=1}^{W} e^{-\beta E_{i}}
$$

Substituindo (1.57) em (1.41), temos que a entropia canônica é dada por

$$
\begin{gathered}
S=-k \sum_{i=1}^{W} p_{i} \ln p_{i}=-k \sum_{i=1}^{W} \frac{e^{-\beta E_{i}}}{Z} \ln \frac{e^{-\beta E_{i}}}{Z} \\
=k \sum_{i=1}^{W} \frac{e^{-\beta E_{i}}}{Z} \ln Z-k \sum_{i=1}^{W} \frac{e^{-\beta E_{i}}}{Z} \ln e^{-\beta E_{i}} \\
=k \frac{Z}{Z} \ln Z+k \beta \sum_{i=1}^{W} p_{i} E_{i} \\
\therefore S=k[\ln Z+\beta\langle E\rangle] .
\end{gathered}
$$

com $\langle E\rangle$ sendo a energia média do sistema, que pode ser expressa como

$$
\langle E\rangle=\sum_{i=1}^{W} p_{i} E_{i}=\sum_{i=1}^{W} \frac{e^{-\beta E_{i}}}{Z} E_{i}=-\frac{\partial \ln Z}{\partial \beta} .
$$

No ensemble canônico, como o sistema está em equilíbrio térmico com o reservatório, a energia pode variar, mas a temperatura $T$, o volume $V$ e o número de constituintes $N$ do sistema são fixos, ou seja, $S=S_{N}(T, V)$. Escrevendo (1.59) como 


$$
-\frac{1}{\beta} \ln Z=\langle E\rangle-\frac{1}{k \beta} S
$$

e comparando com (1.12), $F=E-T S$, podemos inferir que existe uma correspondência entre as grandezas estatísticas e termodinâmicas, [16], do seguinte modo

$$
\begin{gathered}
F=-\frac{1}{\beta} \ln Z, \\
\beta=\frac{1}{k T} .
\end{gathered}
$$

A função de partição canônica para sistemas contínuos é uma função da temperatura e do volume, dada por, [16],

$$
Z_{N}(T, V)=\frac{1}{h^{d N} N !} \int \exp \left(-\frac{H_{N}(\mathbf{p}, \mathbf{q})}{k T}\right) d \mathbf{p} d \mathbf{q}
$$

com $H_{N}(\mathbf{p}, \mathbf{q})$ sendo a hamiltoniana de um sistema composto de $N$ partículas, $h$ a constante de Plank e $d$ a dimensão do sistema. O somatório em (1.58) é feito sobre todos os microestados acessíveis ao sistema e o número microestados que possuem a mesma energia $E$ é dado por $W_{N}(E, V)$. Então, podemos substituir o somatório sobre todos os microestados por um somatório sobre a energia:

$$
Z_{N}(T, V)=\sum_{E} W_{N}(E, V) \exp \left(-\frac{E}{k T}\right)
$$

No caso de sistemas contínuos,

$$
Z_{N}(T, V)=\frac{1}{\epsilon_{0}} \int_{0}^{\infty} W_{N}(E, V) \exp \left(-\frac{E}{k T}\right) d E
$$

As expressões (1.65) e (1.66) mostram que as funções de partição canônica e microcanônica relacionam-se por uma transformada de Laplace, ou seja, uma vez obtida a função de partição microcanônica podemos, através de uma transformada de Laplace, obter a função de partição canônica ou obtida a função de partição canônica, via transformada inversa de Laplace, obtemos a função de partição microcanônica [16],

$$
W_{N}(E, V)=\frac{\epsilon_{0}}{2 \pi i} \int_{\frac{1}{k T^{\prime}}-i \infty}^{\frac{1}{k T^{\prime}}+i \infty} Z_{N}(T, V) \exp \left(\frac{E}{k T}\right) d\left(\frac{1}{k T}\right), \frac{1}{k T^{\prime}}>0
$$


Uma forma alternativa de se obter os ensembles estatísticos é através da maximização do número de microestados do sistema composto $\mathcal{W}$ (Figura 1.1), apresentado na seção 1.3. Obter os $n_{i}^{*}$ da expressão (1.44) é equivalente a obter os $p_{i}$ da expressão (1.48), ou seja, temos que maximizar (1.48), dado os vínculos de cada ensemble, utilizando multiplicadores de Lagrange.

- Ensemble Microcanônico

No caso do ensemble microcanônico, todos os $\mathcal{N}$ sistemas estão isolados, ou seja, energia $E$, volume $V$ e número de constituintes $N$ são fixos. Portanto, o único vínculo é a normalização dos $p_{i}$ :

$$
\sum_{i=1}^{W} p_{i}=1 .
$$

Maximizando (1.41) com o vínculo (1.68), temos que

$$
\begin{gathered}
-\sum_{i=1}^{W} \delta\left(p_{i} \ln p_{i}\right)-\alpha \sum_{i=1}^{W} \delta p_{i}=-\sum_{i=1}^{W} \delta p_{i}\left[\ln p_{i}+1\right]-\alpha \sum_{i=1}^{W} \delta p_{i}= \\
=-\sum_{i=1}^{W} \delta p_{i}\left[\ln p_{i}+1+\alpha\right]=0, \\
\therefore\left[\ln p_{i}+1+\alpha\right]=0 \rightarrow p_{i}=e^{-1-\alpha},
\end{gathered}
$$

com $\alpha$ sendo um multiplicador de Lagrange. Aplicando a normalização em (1.69), ficamos com

$$
\begin{gathered}
\sum_{i=1}^{W} p_{i}=\sum_{i=1}^{W} e^{-1-\alpha}=W e^{-1-\alpha}=1 \rightarrow e^{-1-\alpha}=\frac{1}{W} \\
\therefore p_{i}=\frac{1}{W}
\end{gathered}
$$

que é o mesmo resultado de (1.49). 
- Ensemble Canônico

No caso do ensemble canônico, todos os $\mathcal{N}$ sistemas estão em equilíbrio térmico, as energias dos sistemas podem variar, mas temperatura $T$, volume $V$ e número de constituintes $N$ são fixos. O sistema composto está isolado, portanto sua energia $\mathcal{E}$ é conservada. Seja $E_{i}$ a energia de um sistema no microestado $i$, no caso em que a energia é aditiva,

$$
\sum_{i=1}^{W} n_{i}^{*} E_{i}=\mathcal{E}=\mathcal{N}\langle E\rangle,
$$

com $\langle E\rangle$ sendo a energia média dos sistemas. Com isso, além do vínculo da normalização (1.68), temos também

$$
\sum_{i=1}^{W} p_{i} E_{i}=\langle E\rangle
$$

Maximizando (1.41) com os vínculos (1.68) e (1.73), temos que

$$
\begin{aligned}
&-\sum_{i=1}^{W} \delta\left(p_{i} \ln p_{i}\right)-\alpha \sum_{i=1}^{W} \delta p_{i}-\beta \sum_{i=1}^{W} \delta\left(p_{i} E_{i}\right)=-\sum_{i=1}^{W} \delta p_{i}\left[\ln p_{i}+1\right]-\alpha \sum_{i=1}^{W} \delta p_{i}- \\
&-\beta \sum_{i=1}^{W} \delta p_{i} E_{i} \\
&=-\sum_{i=1}^{W} \delta p_{i}\left[\ln p_{i}+1+\alpha+\beta E_{i}\right]=0, \\
& \therefore\left[\ln p_{i}+1+\alpha+\beta E_{i}\right]=0 \rightarrow p_{i}=e^{-1-\alpha} e^{-\beta E_{i}},
\end{aligned}
$$

com $\alpha$ e $\beta$ sendo multiplicadores de Lagrange. Aplicando a normalização em (1.74), encontramos

$$
\sum_{i=1}^{W} p_{i}=\sum_{i=1}^{W} e^{-1-\alpha} e^{-\beta E_{i}}=e^{-1-\alpha} \sum_{i=1}^{W} e^{-\beta E_{i}}=1 \rightarrow e^{-1-\alpha}=\frac{1}{Z}
$$

sendo o multiplicador de Lagrange $\beta=\frac{1}{k T}$, temos o mesmo resultado de (1.57):

$$
\therefore p_{i}=\frac{e^{-\beta E_{i}}}{Z} \text {. }
$$




\title{
Capítulo 2
}

\section{Sistemas com Interações de Longo}

\begin{abstract}
Alcance
No Capítulo 1 são apresentados os ensembles estatísticos, em especial o canônico e o microcanônico, e como eles se relacionam entre si e com os potenciais termodinâmicos. Entretanto, em sistemas com interações de longo alcance, os ensembles nem sempre são equivalentes e faz-se necessário o uso do ensemble microcanônico, por ser o ensemble relacionado a sistemas isolados. Neste capítulo, vemos uma definição de interações de longo alcance e como a principal característica de sistemas desse tipo, a não-aditividade, leva à inequivalência entre os ensembles microcanônico e canônico. Na última seção são apresentados alguns exemplos de sistemas com interações de longo alcance.
\end{abstract}

\subsection{Definição}

Para definirmos uma interação de longo alcance, vamos calcular a energia potencial $u$ de uma partícula localizada no centro de uma esfera de raio $R$, onde todas as outras partículas estão homogeneamente distribuídas (Figura 2.1), [6]. A longas distâncias, a interação de pares das partículas é dado por um potencial do tipo

$$
V(r)=\frac{J}{r^{\alpha}} .
$$

com $r$ sendo a distância entre as partículas, $J$ e $\alpha$ constantes. Exluindo a contribuição para a energia $u$ vinda de partículas localizadas a curtas distâncias $r<\delta$, uma vez que o interesse é a natureza de longo alcance do potencial e para evitar a divergência a curtas distâncias, temos que, em $d$ dimensões 


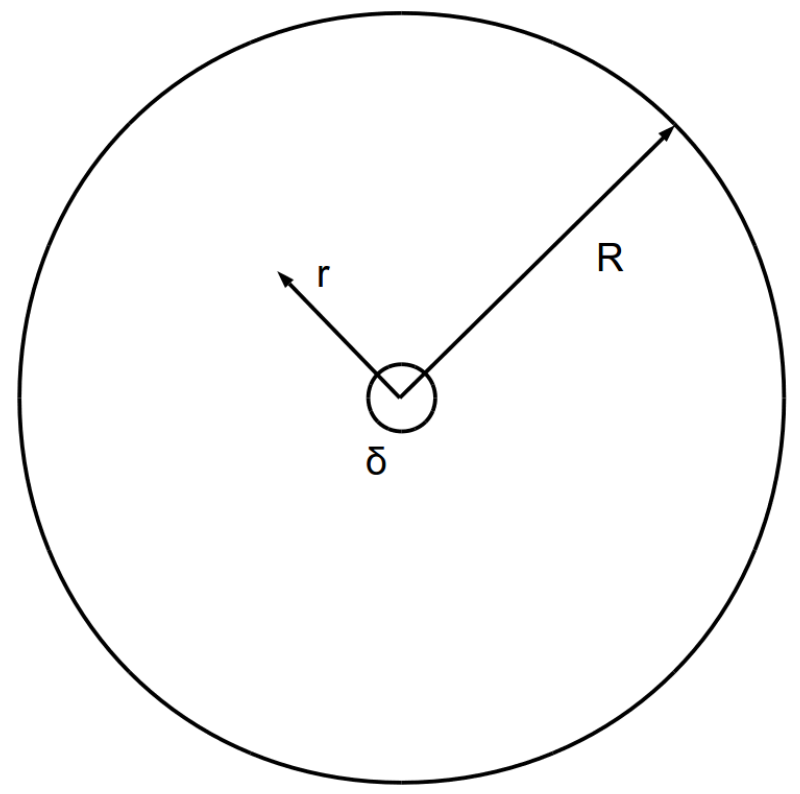

Figura 2.1: Partícula localizada no centro de uma esfera com distribuição homogênea de partículas. O raio da esfera exterior é $R$ e da esfera interior é $\delta$.

$$
\begin{gathered}
u=\int_{\delta}^{R} \rho \frac{J}{r^{\alpha}} d^{d} r= \\
=\rho J \Omega_{d} \int_{\delta}^{R} r^{d-\alpha-1} d^{d} r= \\
=\left.\frac{\rho J \Omega_{d}}{d-\alpha} r^{d-\alpha}\right|_{\delta} ^{R} \\
\therefore u=\frac{\rho J \Omega_{d}}{d-\alpha}\left[R^{d-\alpha}-\delta^{d-\alpha}\right], \quad \text { se } \alpha \neq d,
\end{gathered}
$$

onde $\rho$ é uma densidade qualquer e $\Omega_{d}$ é o volume angular em $d$ dimensões. Quando $\alpha=d$, temos

$$
\begin{aligned}
u & =\int_{\delta}^{R} \rho \frac{J}{r^{\alpha}} d^{d} r= \\
& =\rho J \Omega_{d} \int_{\delta}^{R} r^{-1} d^{d} r= \\
& =\left.\rho J \Omega_{d} \ln r\right|_{\delta} ^{R} \\
\therefore u & =\rho J \Omega_{d} \ln \left(\frac{R}{\delta}\right), \quad \text { se } \alpha=d .
\end{aligned}
$$


Com isso, podemos escrever

$$
u=\left\{\begin{array}{cc}
\frac{\rho J \Omega_{d}}{d-\alpha}\left[R^{d-\alpha}-\delta^{d-\alpha}\right], & \alpha \neq d . \\
\rho J \Omega_{d} \ln \left(\frac{R}{\delta}\right), & \alpha=d .
\end{array}\right.
$$

De (2.4), se $\alpha>d$ então a energia potencial é finita quando $R \rightarrow \infty$, neste caso a energia cresce linearmente com o tamanho do sistema e dizemos que a interação é de curto alcance. Se $\alpha \leq d$ a energia potencial diverge quando $R \rightarrow \infty$, ou seja, a energia cresce superlinearmente com o tamanho do sistema e a interação é dita ser de longo alcance, [6]. Sistemas com interações de longo alcance podem ser encontrados em diversas áreas da física, como astrofísica, física de plasma, hidrodinâmica, física atômica e nuclear. Sistemas desse tipo podem apresentar diversas peculiaridades, como calor específico negativo, susceptibilidade negativa, inequivalência entre ensembles e quebra de ergodicidade.

\subsection{Aditividade e Não-Aditividade}

Sistemas com interações de longo alcance são intrinsecamente não-aditivos. Essa é a principal diferença entre sistemas com interações de longo alcance e sistemas com interações de curto alcance. Para entender melhor a aditividade, seja um sistema dividido em dois subsistemas, 1 e 2 , com energia total

$$
E_{\text {total }}=E_{1}+E_{2}+E_{\text {int }}
$$

com $E_{1}, E_{2}$ e $E_{\text {int }}$ sendo a energia do subsistema 1, a energia do subsistema 2 e a energia de interação entre os subsistemas, respectivamente. No caso em que as interações são de curto alcance, existem mais interações entre partículas de um mesmo subsistema do que partículas de subsistemas diferentes (como ilustrado na figura 2.2(a), onde as linhas sólidas azuis representam interações entre partículas de um mesmo subsistema e as linhas tracejadas vermelhas representam interações entre partículas de subsistemas diferentes). É fácil notar que, quando o número de partículas cresce $(N \rightarrow \infty)$, o número de linhas sólidas azuis se torna muito maior que o número de linhas tracejadas vermelhas, pois a interação entre os subsistemas fica restrita às partículas que estão próximas a barreira que os separa. Com isso, podemos dizer que no limite termodinâmico $(N \rightarrow \infty) E_{\text {int }} \ll E_{1}+E_{2}$, ou seja, 


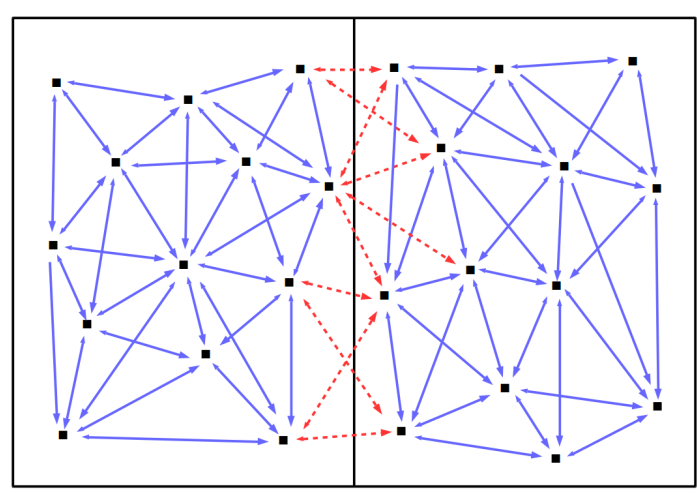

(a) Interação de Curto Alcance

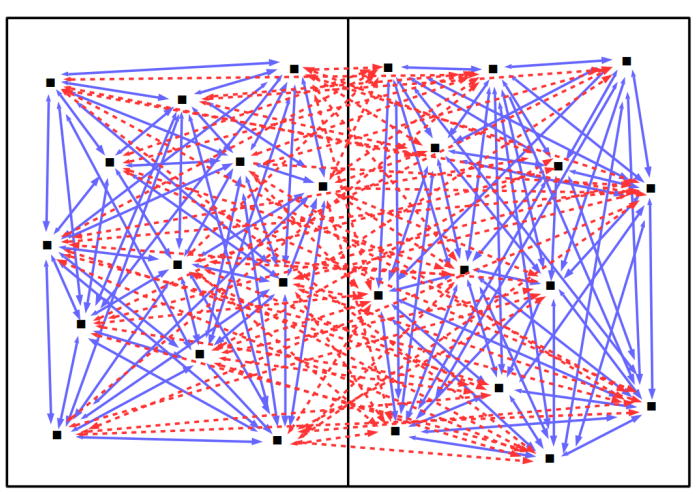

(b) Interação de Longo Alcance

Figura 2.2: Representação de um sistema dividido em dois subsistemas que interagem entre si através de (a) interações de curto alcance e (b) interações de longo alcance. As linhas sólidas azuis representam interações entre partículas do mesmo subsistema e as linhas tracejadas vermelhas representam interações entre partículas de subsistemas diferentes.

$$
E_{\text {total }} \simeq E_{1}+E_{2}
$$

Como a energia do sistema é dada pela soma das energias de cada subsistema, dizemos que a energia é aditiva. Quando as interações são de longo alcance, o número de linhas tracejadas vermelhas é proporcional ao número de linhas sólidas azuis, Figura 2.2(b), porque a interação entre os subsistemas não fica restrita às partículas próximas à barreira. Mesmo no limite termodinâmico, a energia de interação tem a mesma ordem de grandeza das energias dos subsistemas, $E_{\text {int }} \sim E_{1}+E_{2}$, ou seja,

$$
E_{\text {total }} \neq E_{1}+E_{2}
$$

Como a energia do sistema não é dada pela soma das energias dos subsistemas, dizemos que a energia não é aditiva. Se a energia de um sistema for aditiva, reduzir pela metade o tamanho do sistema implica em reduzir pela metade a energia do sistema, ou seja, se um sistema for aditivo ele será necessariamente extensivo, [6]:

$$
\text { Aditividade } \Rightarrow \text { Extensividade, }
$$

logo, também vale que:

$$
\text { Não-extensividade } \Rightarrow \text { Não-aditividade. }
$$

Sistemas com interação de longo alcance geralmente são não-aditivos e não-extensivos. Entretanto, a extensividade pode ser recuperada através da prescrição de Kac, [6]. A 
prescrição de Kac é um artifício matemático de renormalização do potencial do sistema, com a finalidade de tornar a energia potencial proporcional ao tamanho do sistema, assim como é a energia cinética. Porém, mesmo recuperando a extensividade de um sistema, ele continua sendo não-aditivo.

Um exemplo de sistema extensivo e não-aditivo, que é visto com mais detalhe no Capítulo 4, é o modelo de Curie-Weiss para o estudo de sistemas magnéticos, [6]. A Hamiltoniana de Curie-Weiss tem a seguinte forma

$$
H_{C W}=-\frac{J}{2 N} \sum_{i, j=1}^{N} S_{i} S_{j}=-\frac{J}{2 N}\left(\sum_{i=1}^{N} S_{i}\right)^{2}
$$

onde os spins podem assumir os valores $S_{i}= \pm 1$ e $J$ é uma constante de acoplamento. O fator $\frac{1}{N}$ é a prescrição de Kac, com isso a energia é proporcional ao número de partículas $N$ e o sistema é extensivo. Cada spin está situado em um sítio e interage com todos os outros spins, ou seja, a interação não decai com a distância $(\alpha=0)$. Uma forma de escrever (2.8) é utilizando a magnetização do sistema,

$$
M=N m=\sum_{i=1}^{N} S_{i},
$$

onde $M$ e $m$ são as magnetizações extensiva e intensiva, respectivamente. Com isso, a hamiltoniana de Curie-Weiss se torna

$$
H_{C W}=-\frac{J}{2 N} M^{2}=-\frac{J N}{2} m^{2}
$$

a expressão (2.10) é proporcional a $N$, ou seja, extensiva. Se um sistema for dividido em dois subsistemas ( 1 e 2 ) de tal forma que as $\frac{N}{2}$ partículas do subsistema 1 estão com spin para cima e as outras $\frac{N}{2}$ partículas do subsistema 2 estão com spin para baixo, como ilustrado na figura 2.3, então as energias $E_{1}$ e $E_{2}$ (correspondente aos subsistemas 1 e 2 , respectivamente) serão, de (2.10)

$$
E_{1}=E_{2}=-\frac{J N}{4}
$$

Entretanto, calculando a energia total do sistema encontramos $E=0$. Como $E \neq E_{1}+E_{2}$, o sistema não é aditivo. É interessante notar que, de (2.5),

$$
E_{\text {int }}=E-E_{1}-E_{2}=\frac{J N}{2}
$$


ou seja, $E_{\text {int }}$ cresce com $N$. Mesmo no limite termodinâmico o sistema continua sendo não-aditivo.

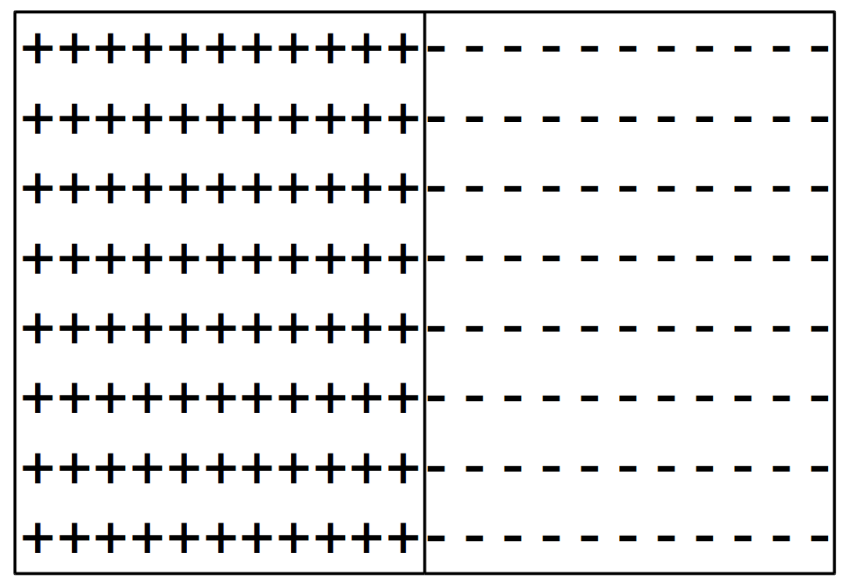

Figura 2.3: Representação de um sistema de spins dividido em dois subsistemas com $\frac{N}{2}$ spins em cada um. No subsistema 1 todos os spins estão para cima e no subsistema 2 todos os spins estão para baixo.

\subsection{Equivalência e Inequivalência de Ensemble}

Em sistemas com interações de curto alcance, os ensembles estatísticos são equivalentes. A equivalência entre os ensembles possui duas propriedades importantes, [6]:

(i) No limite termodinâmico, as flutuações dos parâmetros termodinâmicos que não estão fixados desaparecem;

(ii) Um macroestado que pode ser realizado em um ensemble também pode ser realizado em outro ensemble.

Por exemplo, no caso da equivalência entre os ensembles microcanônico e canônico: um sistema isolado com uma energia fixa possui uma temperatura média. Se um mesmo sistema for colocado em contato térmico com um reservatório a essa mesma temperatura, então ele terá uma energia média igual a energia do sistema isolado. Portanto, existe uma correspondência um-para-um entre a energia e a temperatura. A vantagem da equivalência entre ensembles é a liberdade de poder escolher o ensemble onde os cálculos são mais fáceis e extrair dele toda a termodinâmica do sistema. Utilizando as expressões (1.40) e (1.62) em (1.65), [9], temos 


$$
\begin{aligned}
& Z_{N}(T)=\exp \left(-\frac{F_{N}(T)}{T}\right)=\sum_{E} W_{N}(E) \exp \left(-\frac{E}{T}\right)= \\
&=\sum_{E} \exp \left(S_{N}(E)\right) \exp \left(-\frac{E}{T}\right) \\
& \therefore \exp \left(-\frac{F_{N}(T)}{T}\right)=\sum_{E} \exp \left[-\frac{1}{T}\left(E-T S_{N}(E)\right)\right]
\end{aligned}
$$

onde a constante de Boltzmann $k$ é fixada como sendo 1 daqui para frente. No equilíbro, o valor do somatório em (2.13) pode ser aproximado pelo maior termo da soma, com isso

$$
F_{N}(T) \simeq \min _{E}\left[E-T S_{N}(E)\right]
$$

Para satisfazer a condição de mínimo em (2.14), devemos ter

$$
\begin{gathered}
\frac{\partial}{\partial E}\left[E-T S_{N}(E)\right]=0 \rightarrow 1-T \frac{\partial S_{N}}{\partial E}=0 \rightarrow \frac{\partial S_{N}}{\partial E}=\frac{1}{T}, \\
\frac{\partial^{2}}{\partial E^{2}}\left[E-T S_{N}(E)\right]>0 \rightarrow-T \frac{\partial^{2} S_{N}}{\partial E^{2}}>0 \rightarrow \frac{\partial^{2} S_{N}}{\partial E^{2}}<0 .
\end{gathered}
$$

Quando as condições (2.15) e (2.16) são satisfeitas, temos

$$
F_{N}(T) \simeq E-T S_{N}(E)
$$

recuperando a transformada de Legendre (1.12). Ou seja, para existir equivalência entre os ensembles canônico e microcanônico, a entropia $S$ deve ser uma função côncava da energia $E$, [6]. Quando a entropia de um sistema isolado possui uma região convexa, como a região $\left[E_{1}, E_{2}\right]$ da linha sólida da figura $2.4($ a), é possível substituir a região convexa por um "envelope côncavo", [6]. Das equações (1.2)(a) e (1.63),

$$
S\left(E_{2}\right)=S\left(E_{1}\right)+\int_{E_{1}}^{E_{2}} \beta(E) d E,
$$

onde $\beta(E)$ está representado na figura 2.4(b). A integração em (2.18) pode ser realizada sobre linha tracejada da figura 2.4(b), com isso, temos que

$$
S\left(E_{2}\right)=S\left(E_{1}\right)+\left(E_{2}-E_{1}\right) \beta_{T}
$$




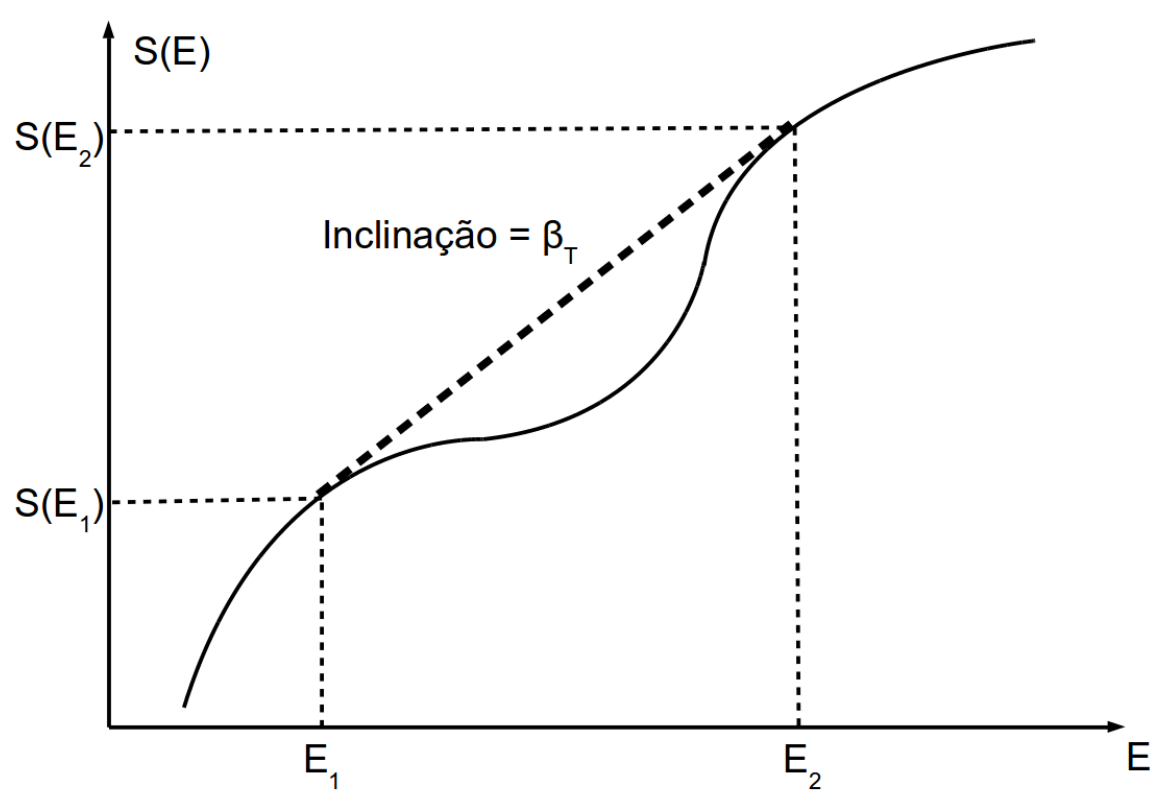

(a) Entropia vs. energia

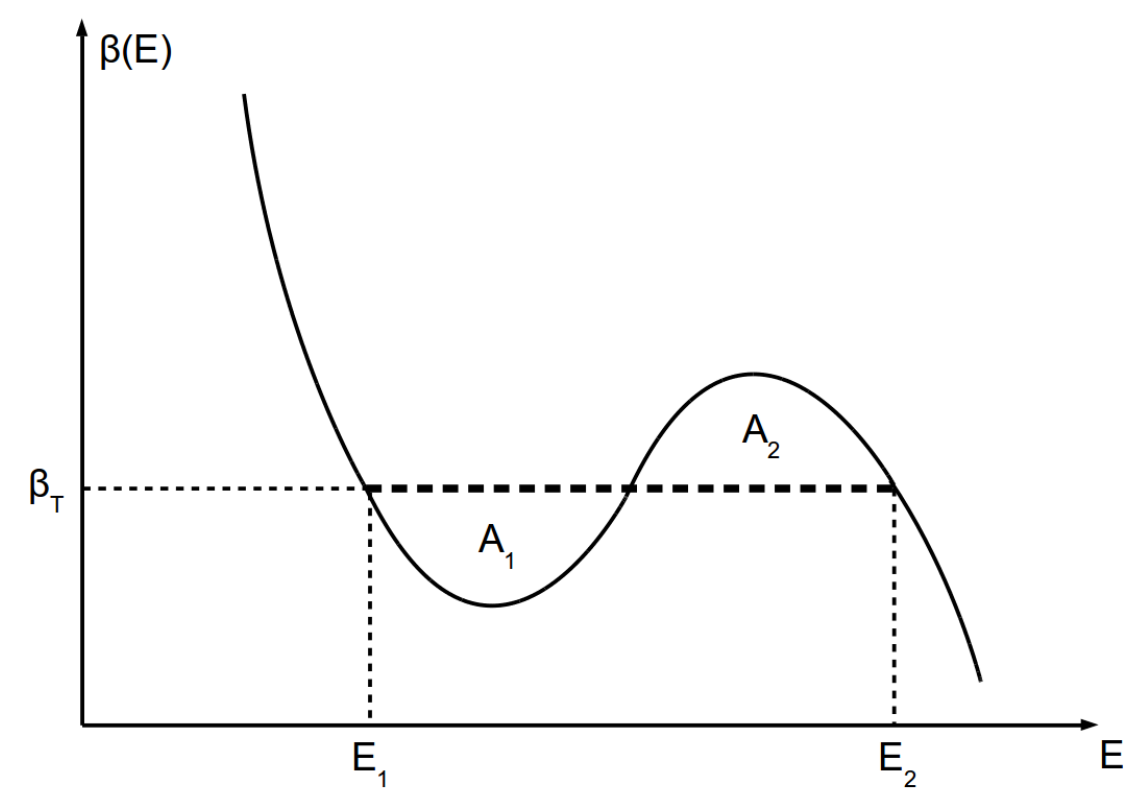

(b) Inverso da temperatura vs. energia

Figura 2.4: (a) Representação da entropia $S$ em função da energia $E$ (linha sólida) com uma região convexa, $\left[E_{1}, E_{2}\right]$, e um envelope côncavo (linha tracejada). (b) Representação do inverso da temperatura $\beta$ em função da energia $E$ (linha sólida). 
A condição para que (2.19) seja igual a (2.18) é que as áreas $A_{1}$ e $A_{2}$, na figura 2.4(b), sejam iguais. Integrando sobre a linha tracejada da figura $2.4(\mathrm{~b})$, de $E_{1}$ até um valor qualquer de $E \in\left[E_{1}, E_{2}\right]$, temos que a equação da reta tracejada com inclinação $\beta_{T}$ (Figura 2.4(a)) é dada por

$$
S(E)=S\left(E_{1}\right)+\left(E-E_{1}\right) \beta_{T}, \quad E \in\left[E_{1}, E_{2}\right] .
$$

Precisamos saber qual o significado físico desta reta. Para isso, considere que o sistema se separe em duas fases, uma com energia $E_{1}$ e a outra com energia $E_{2}$, então a entropia total será

$$
S=(1-\lambda) S\left(E_{1}\right)+\lambda S\left(E_{2}\right)
$$

onde $\lambda \in[0,1]$ é a fração do sistema com energia $E_{2}$. No caso em que a energia também é aditiva, a energia total do sistema é expressa como

$$
E=(1-\lambda) E_{1}+\lambda E_{2}
$$

Substituindo (2.19) em (2.21), temos

$$
S=S\left(E_{1}\right)+\lambda\left(E_{2}-E_{1}\right) \beta_{T}
$$

Da equação (2.22), podemos escrever (2.23) como

$$
S(E)=S\left(E_{1}\right)+\left(E-E_{1}\right) \beta_{T}, \quad E \in\left[E_{1}, E_{2}\right],
$$

que é a mesma expressão para a reta (2.20). Ou seja, a reta tracejada da figura 2.4(a) corresponde a uma separação de fases no sistema. Como a entropia da separação de fases é maior, o sistema tende a se separar nas duas fases. Neste caso, a região convexa é substituída pela reta tracejada (Figura 2.4(a)), recuperando a equivalência entre os ensembles. Esse procedimento, de construção de um "envelope côncavo", é chamado de construção de Maxwell.

Sistemas com interações de longo alcance possuem energia não-aditiva, mesmo no limite termodinâmico. Portanto, não é possível existir uma separação de fases, pois a equação (2.22) deixa de ser válida. Neste caso, quando não é possível recuperar a equivalência entre os ensembles, fica claro que não há uma correspondência um-para-um entre 
energia e temperatura (linha sólida da figura 2.4(b)). Das equações (1.2) e (1.24),

$$
\frac{\partial^{2} S}{\partial E^{2}}=-\frac{1}{T^{2} C}
$$

ou seja, nas regiões onde a entropia é uma função convexa da energia, o calor específico $C$ é negativo. Resumindo, quando um sistema possui energia aditiva, a construção de Maxwell garante a equivalência entre os ensembles, porém quando o sistema possui energia não-aditiva e calor específico negativo, os ensembles microcanônico e canônico são inequivalentes (Figura 2.5).

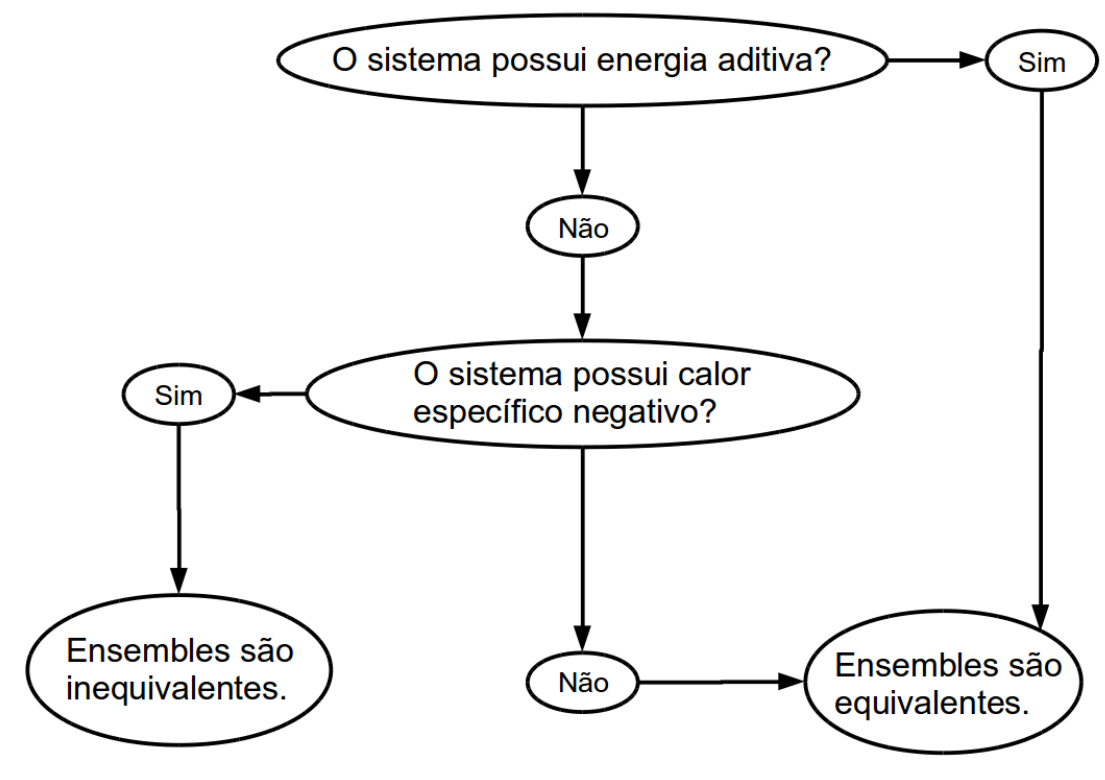

Figura 2.5: Fluxograma que ilustra quando temos equivalência e inequivalência entre os ensembles canônico e microcanônico.

Quando um sistema não possui energia aditiva, como sistemas com interações de longo alcance, é recomendado o uso do ensemble microcanônico, pois as construções do ensemble canônico exigem a aditividade da energia (equações (1.52) e (1.72)).

\subsection{Exemplos}

Nesta seção são apresentados alguns exemplos de sistemas com interações de longo alcance, que são usados para análise dos métodos computacionais no Capítulo 4. O modelo HMF-1D é resolvido analiticamente e numericamente, mas o modelo HMF-2D apenas numericamente. 


\section{- HMF-1D}

A Hamiltoniana de Campo Médio unidimensional (HMF-1D) é definida como, [1]:

$$
H_{N}=\sum_{i=1}^{N} \frac{p_{i}^{2}}{2}+\frac{J}{2 N} \sum_{i, j=1}^{N}\left[1-\cos \left(\theta_{i}-\theta_{j}\right)\right],
$$

onde $\theta_{i} \in[0,2 \pi)$ é a posição da partícula $i$ de massa unitária, $p_{i}$ é o momento conjugado correspondente e o fator $\frac{1}{N}$ é a prescrição de Kac. Esse sistema pode ser interpretado como partículas movendo-se em um círculo de raio unitário interagindo por um potencial, atrativo $(J>0)$ ou repulsivo $(J<0)$, de alcance infinito, [6]. Neste trabalho é estudado o caso atrativo $(J=1)$. O modelo HMF-1D possui diversas propriedades térmicas e dinâmicas que aparecem em outros sistemas com interações de longo alcance e, por conta disso, é utilizado como um modelo simplificado para alguns sistemas físicos reais. A hamiltoniana (2.26) pode ser reescrita da seguinte maneira

$$
\begin{aligned}
H_{N} & =\sum_{i=1}^{N} \frac{p_{i}^{2}}{2}+\frac{1}{2 N} \sum_{i, j=1}^{N}\left[1-\cos \left(\theta_{i}-\theta_{j}\right)\right]= \\
& =\sum_{i=1}^{N} \frac{p_{i}^{2}}{2}+\frac{1}{2 N} \sum_{i, j=1}^{N}\left[1-\cos \theta_{i} \cos \theta_{j}-\sin \theta_{i} \sin \theta_{j}\right]= \\
& =\sum_{i=1}^{N} \frac{p_{i}^{2}}{2}+\frac{1}{2 N}\left[N^{2}-\sum_{i, j=1}^{N} \cos \theta_{i} \cos \theta_{j}-\sum_{i, j=1}^{N} \sin \theta_{i} \sin \theta_{j}\right]= \\
& =\sum_{i=1}^{N} \frac{p_{i}^{2}}{2}+\frac{N}{2}\left[1-\left(\sum_{i=1}^{N} \frac{\cos \theta_{i}}{N}\right)^{2}-\left(\sum_{i=1}^{N} \frac{\sin \theta_{i}}{N}\right)^{2}\right] .
\end{aligned}
$$

Definindo as componentes $x$ e $y$ da magnetização intensiva como

$$
m_{x}=\frac{1}{N} \sum_{i=1}^{N} \cos \theta_{i} \quad e \quad m_{y}=\frac{1}{N} \sum_{i=1}^{N} \sin \theta_{i},
$$

a Hamiltoniana de Campo Médio fica

$$
H_{N}=\sum_{i=1}^{N} \frac{p_{i}^{2}}{2}+\frac{N}{2}\left[1-m^{2}\right]
$$

com $m^{2}=m_{x}^{2}+m_{y}^{2}$. A solução analítica de equilíbrio para esse sistema é conhecida e obtida no Capítulo 4, junto com resultados numéricos. 
- $\mathrm{HMF}-2 \mathrm{D}$

A Hamiltoniana de Campo Médio bidimensional (HMF-2D) é definida como, [3]:

$$
\begin{aligned}
H_{N}= & \sum_{i=1}^{N} \frac{p_{i \theta}^{2}+p_{i \phi}^{2}}{2}+\frac{1}{2 N} \sum_{i, j=1}^{N}\left[c_{1}+c_{2}+d-c_{1} \cos \left(\theta_{i}-\theta_{j}\right)-\right. \\
& \left.-c_{2} \cos \left(\phi_{i}-\phi_{j}\right)-d \cos \left(\theta_{i}-\theta_{j}\right) \cos \left(\phi_{i}-\phi_{j}\right)\right],
\end{aligned}
$$

para cada partícula $i, p_{i \theta}$ é o momento conjugado a $\theta_{i}, p_{i \phi}$ é o momento conjugado a $\phi_{i}$ e $c_{1}, c_{2}$ e $d$ são constantes. Podemos reescrever a energia potencial em (2.29) como

$$
\begin{aligned}
U= & \frac{1}{2 N} \sum_{i, j=1}^{N}\left[c_{1}+c_{2}+d-c_{1} \cos \left(\theta_{i}-\theta_{j}\right)-\right. \\
& \left.-c_{2} \cos \left(\phi_{i}-\phi_{j}\right)-d \cos \left(\theta_{i}-\theta_{j}\right) \cos \left(\phi_{i}-\phi_{j}\right)\right]= \\
= & \frac{1}{2 N} \sum_{i, j=1}^{N}\left[c_{1}+c_{2}+d-c_{1}\left(\cos \theta_{i} \cos \theta_{j}+\sin \theta_{i} \sin \theta_{j}\right)-\right. \\
& -c_{2}\left(\cos \phi_{i} \cos \phi_{j}+\sin \phi_{i} \sin \phi_{j}\right)- \\
& \left.-d\left(\cos \theta_{i} \cos \theta_{j}+\sin \theta_{i} \sin \theta_{j}\right)\left(\cos \phi_{i} \cos \phi_{j}+\sin \phi_{i} \sin \phi_{j}\right)\right]= \\
= & \frac{1}{2 N}\left\{\sum_{i, j=1}^{N}\left(c_{1}+c_{2}+d\right)-c_{1}\left[\left(\sum_{i=1}^{N} \cos \theta_{i}\right)^{2}+\left(\sum_{i=1}^{N} \sin \theta_{i}\right)^{2}\right]-\right. \\
& -c_{2}\left[\left(\sum_{i=1}^{N} \cos \phi_{i}\right)^{2}+\left(\sum_{i=1}^{N} \sin \phi_{i}\right)^{2}\right]- \\
& -d \sum_{i, j=1}^{N} \underbrace{\left(\cos \theta_{i} \cos \theta_{j}+\sin \theta_{i} \sin \theta_{j}\right)\left(\cos \phi_{i} \cos \phi_{j}+\sin \phi_{i} \sin \phi_{j}\right)}_{A_{i j}}\} .
\end{aligned}
$$

Definindo as magnetizações nas direções $\theta$ e $\phi$ como

$$
\begin{aligned}
& m_{\theta}^{2}=\left(\sum_{i=1}^{N} \frac{\cos \theta_{i}}{N}\right)^{2}+\left(\sum_{i=1}^{N} \frac{\sin \theta_{i}}{N}\right)^{2} \\
& m_{\phi}^{2}=\left(\sum_{i=1}^{N} \frac{\cos \phi_{i}}{N}\right)^{2}+\left(\sum_{i=1}^{N} \frac{\sin \phi_{i}}{N}\right)^{2}
\end{aligned}
$$

respectivamente, ficamos com 


$$
U=\frac{1}{2 N}\left[N^{2}\left(c_{1}+c_{2}+d\right)-N^{2}\left(c_{1} m_{\theta}^{2}+c_{2} m_{\phi}^{2}\right)-d \sum_{i, j=1}^{N} A_{i j}\right]
$$

Temos que

$$
\begin{aligned}
A_{i j}= & \left(\cos \theta_{i} \cos \theta_{j}+\sin \theta_{i} \sin \theta_{j}\right)\left(\cos \phi_{i} \cos \phi_{j}+\sin \phi_{i} \sin \phi_{j}\right)= \\
= & \cos \theta_{i} \cos \phi_{i} \cos \theta_{j} \cos \phi_{j}+\sin \theta_{i} \sin \phi_{i} \sin \theta_{j} \sin \phi_{j}+ \\
& +\cos \theta_{i} \sin \phi_{i} \cos \theta_{j} \sin \phi_{j}+\sin \theta_{i} \cos \phi_{i} \sin \theta_{j} \cos \phi_{j}= \\
= & \frac{1}{2}\left[\cos \left(\theta_{i}+\phi_{i}\right) \cos \left(\theta_{j}+\phi_{j}\right)+\cos \left(\theta_{i}-\phi_{i}\right) \cos \left(\theta_{j}-\phi_{j}\right)+\right. \\
& \left.+\sin \left(\theta_{i}+\phi_{i}\right) \sin \left(\theta_{j}+\phi_{j}\right)+\sin \left(\theta_{i}-\phi_{i}\right) \sin \left(\theta_{j}-\phi_{j}\right)\right] .
\end{aligned}
$$

Realizando a soma sobre $i$ e $j$ em (2.34),

$$
\begin{aligned}
& \sum_{i, j=1}^{N} A_{i j}= \frac{1}{2} \sum_{i, j=1}^{N}\left[\cos \left(\theta_{i}+\phi_{i}\right) \cos \left(\theta_{j}+\phi_{j}\right)+\cos \left(\theta_{i}-\phi_{i}\right) \cos \left(\theta_{j}-\phi_{j}\right)+\right. \\
&\left.+\sin \left(\theta_{i}+\phi_{i}\right) \sin \left(\theta_{j}+\phi_{j}\right)+\sin \left(\theta_{i}-\phi_{i}\right) \sin \left(\theta_{j}-\phi_{j}\right)\right]= \\
&= \frac{1}{2}\left[\left(\sum_{i=1}^{N} \cos \left(\theta_{i}+\phi_{i}\right)\right)^{2}+\left(\sum_{i=1}^{N} \sin \left(\theta_{i}+\phi_{i}\right)\right)^{2}+\right. \\
&\left.+\left(\sum_{i=1}^{N} \cos \left(\theta_{i}-\phi_{i}\right)\right)^{2}+\left(\sum_{i=1}^{N} \sin \left(\theta_{i}-\phi_{i}\right)\right)^{2}\right] \\
& \therefore \sum_{i, j=1}^{N} A_{i j}=\frac{N^{2}}{2}\left[P_{+}^{2}+P_{-}^{2}\right]
\end{aligned}
$$

onde as polarizações $P_{+}$e $P_{-}$são definidas como

$$
\begin{aligned}
& P_{+}^{2}=\left(\sum_{i=1}^{N} \frac{\cos \left(\theta_{i}+\phi_{i}\right)}{N}\right)^{2}+\left(\sum_{i=1}^{N} \frac{\sin \left(\theta_{i}+\phi_{i}\right)}{N}\right)^{2}, \\
& P_{-}^{2}=\left(\sum_{i=1}^{N} \frac{\cos \left(\theta_{i}-\phi_{i}\right)}{N}\right)^{2}+\left(\sum_{i=1}^{N} \frac{\sin \left(\theta_{i}-\phi_{i}\right)}{N}\right)^{2},
\end{aligned}
$$

Substituindo (2.35) em (2.33), temos a energia potencial escrita em termos das magnetiazões e polarizações: 


$$
U=\frac{N}{2}\left[c_{1}+c_{2}+d-c_{1} m_{\theta}^{2}-c_{2} m_{\phi}^{2}-\frac{d}{2}\left(P_{+}^{2}+P_{-}^{2}\right)\right]
$$

Substituindo a expressão (2.38) em (2.29), podemos escrever a hamiltoniana do modelo HMF-2D como

$$
H_{N}=\sum_{i=1}^{N} \frac{p_{i \theta}^{2}+p_{i \phi}^{2}}{2}+\frac{N}{2}\left[c_{1}+c_{2}+d-c_{1} m_{\theta}^{2}-c_{2} m_{\phi}^{2}-\frac{d}{2}\left(P_{+}^{2}+P_{-}^{2}\right)\right]
$$

O modelo HMF-2D apresenta uma transição de fase de segunda ordem com calor específico negativo, [3]. Como o sistema possui energia não-aditiva e calor específico negativo, há inequivalência entre os ensembles canônico e microcanônico (Figura 2.5) e, como o ensemble canônico não é recomendado para sistemas com interações de longo alcance (por não possuírem energia aditiva), faz-se necessário o uso do ensemble microcanônico. Entretanto, calcular analiticamente a função de partição microcanônica para o modelo HMF-2D é um trabalho árduo, em comparação aos modelos de Curie-Weiss e HMF-1D. No Capítulo 4 são apresentados resultados numéricos para o caso completamente atrativo, $c_{1}=c_{2}=d=1,[2,14,19,24]$. 


\section{Capítulo 3}

\section{Método de Monte Carlo Microcanônico}

No Capítulo 2 vimos que nem sempre os ensembles são equivalentes, e que o ensemble microcanônico deve ser usado nestes casos. Contudo, obter a função de partição microcanônica, analiticamente, nem sempre é possível. Por conta disto, o uso de métodos computacionais, para obter a função de partição microcanônica, é essencial para o estudo da termodinâmica de sistemas com interação de longo alcance. Neste capítulo, são introduzidos o método de Monte Carlo microcanônico proposto por John R. Ray, [18], seguindo a mesma ideia do método canônico de Metropolis et al., [15], e o método de Monte Carlo proposto por Fugao Wang e David P. Landau, [25], para calcular a densidade de estados de um sistema.

\subsection{Visão Geral}

Os métodos de Monte Carlo são uma classe de algoritmos computacionais que se baseiam em amostragens aleatórias para obter resultados numéricos. Este tipo de método é utilizado em diversas áreas como física, química e biologia. Os métodos de Monte Carlo variam, mas tendem a seguir o seguinte padrão:

1. Define um domínio de possíveis valores;

2. Gera valores aleatoriamente dentro do domínio a partir de uma distribuição de probabilidades;

3. Realiza os cálculos a partir desses valores.

Simulações computacionais usando métodos de Monte Carlo necessitam de números aleatórios. No entanto, a geração de números aleatórios é impossível de se realizar em 
um computador. Por isso, ao invés de gerar números realmente aleatórios, existem geradores de números pseudo-aleatórios. Um bom gerador de números pseudo-aleatórios deve satisfazer a condição de que os números gerados devem ser uniformemente distribuídos no domínio determinado. Um teste para essa condição é gerar pares de números pseudoaleatórios, $(x, y)$, entre 0 e 1 e verificar se eles preenchem o quadrado de área unitária uniformemente. A figura 3.1 mostra o resultado do teste para o gerador de números pseudo-aleatórios padrão da linguagem $C$.

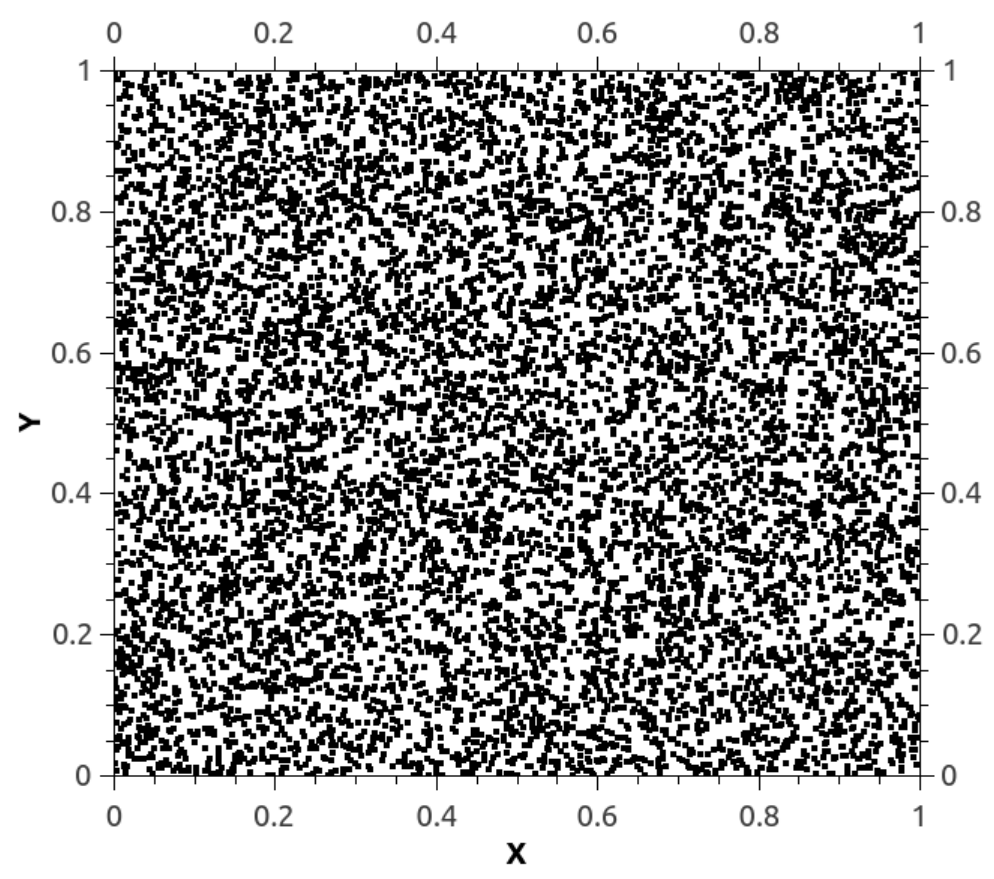

Figura 3.1: Pontos gerados por um gerador de números pseudo-aleatórios.

A motivação para utilizar o método de Monte Carlo em física estatística é o cálculo de integrais de muitas variáveis. Para obter o valor médio de uma grandeza macroscópica $X$, temos que calcular, sobre todo o espaço de fase, o valor médio de $X(\mathbf{p}, \mathbf{q})$ usando a distribuição de probabilidade dos microestados do sistema, $P(\mathbf{p}, \mathbf{q})$ :

$$
<X>=\int X(\mathbf{p}, \mathbf{q}) P(\mathbf{p}, \mathbf{q}) d \mathbf{p} d \mathbf{q}
$$

Uma estimativa do valor da integral em (3.1), utilizando integração de Monte Carlo, é

$$
<X>\simeq \frac{1}{N_{P}} \sum_{i=1}^{N_{P}} X\left(\mathbf{p}_{i}, \mathbf{q}_{i}\right) P\left(\mathbf{p}_{i}, \mathbf{q}_{i}\right),
$$

com $N_{P}$ sendo o número de pontos, $(\mathbf{p}, \mathbf{q})$, gerados no espaço de fase. A principal motivação para utilizar a integração de Monte Carlo vem do fato de que o erro desse método é inversamente proporcional a $N_{P}^{\frac{1}{2}}$, [13], independente da dimensão da integral. 


\subsection{Método de Metropolis}

Ao invés de selecionar um ponto no espaço de fase aleatoriamente e atribuir a ele uma probabilidade $P(\mathbf{p}, \mathbf{q})$, é mais interessante gerar, previamente, pontos com probabilidade $P(\mathbf{p}, \mathbf{q})$. Desta forma, o valor médio de $X$ (equação 3.2 ) é dado por

$$
<X>\simeq \frac{1}{N_{P}} \sum_{i=1}^{N_{P}} X^{*}\left(\mathbf{p}_{i}, \mathbf{q}_{i}\right)
$$

com $X^{*}(\mathbf{p}, \mathbf{q})$ sendo os valores de $X(\mathbf{p}, \mathbf{q})$ para pontos gerados com probabilidade $P(\mathbf{p}, \mathbf{q})$. Uma forma realizar isso é utilizando o algoritmo de Metropolis et al., [15], que é usado para sistemas em contato térmico com um reservatório de energia (ensemble canônico). Neste caso, a probabilidade é dada por

$$
P(\mathbf{p}, \mathbf{q})=\frac{e^{-\beta E(\mathbf{p}, \mathbf{q})}}{Z_{N}(\beta, V)}
$$

$\operatorname{com} \beta=\frac{1}{T}$ e $Z_{N}(\beta, V)$ sendo a função de partição canônica. Computacionalmente, realizar a integração nos momentos é impossível, uma vez que $p \in(-\infty, \infty)$, mas a integração nas posições é possível, pois $q \in V$. Portanto, devemos ter uma probabilidade que dependa apenas de $\mathbf{q}$,

$$
\begin{aligned}
P(\mathbf{q}) & =\int P(\mathbf{p}, \mathbf{q}) d \mathbf{p}=\frac{1}{Z_{N}(\beta, V)} \int e^{-\beta E(\mathbf{p}, \mathbf{q})} d \mathbf{p}= \\
& =\frac{1}{Z_{N}(\beta, V)} \int e^{-\beta[K(\mathbf{p})+U(\mathbf{q})]} d \mathbf{p}= \\
& =\frac{e^{-\beta U(\mathbf{q})}}{Z_{N}(\beta, V)} \int d \mathbf{p} \exp \left(-\beta \sum_{i=1}^{N} \frac{p_{i}^{2}}{2 m}\right)= \\
& =\frac{e^{-\beta U(\mathbf{q})}}{Z_{N}(\beta, V)} \int d \mathbf{p} \prod_{i=1}^{N} \exp \left(-\beta \frac{p_{i}^{2}}{2 m}\right)= \\
& =\frac{e^{-\beta U(\mathbf{q})}}{Z_{N}(\beta, V)}\left[\int_{-\infty}^{\infty} d p \exp \left(-\beta \frac{p^{2}}{2 m}\right)\right]^{d N} .
\end{aligned}
$$

Usando $\int_{-\infty}^{\infty} e^{-\alpha x^{2}} d x=\sqrt{\frac{\pi}{\alpha}}$ em $(3.5)$, ficamos com

$$
P(\mathbf{q})=\left(\frac{2 m \pi}{\beta}\right)^{\frac{d N}{2}} \frac{e^{-\beta U(\mathbf{q})}}{Z_{N}(\beta, V)}=f(\beta, V, N) e^{-\beta U(\mathbf{q})} .
$$

Com a expressão (3.6), o algoritmo de Metropolis funciona da seguinta forma: 
1. Define uma condição inicial para o sistema, q, com energia potencial $U(\mathbf{q})$;

2.1. Muda aleatoriamente a posição de uma partícula, $\mathbf{q} \rightarrow \mathbf{q}^{\prime}$, e computa a nova energia potencial $U\left(\mathbf{q}^{\prime}\right)$;

2.2. Aceita a nova configuração com a probabilidade:

$$
A\left(\mathbf{q} \rightarrow \mathbf{q}^{\prime}\right)=\min \left(1, \frac{e^{-\beta U\left(\mathbf{q}^{\prime}\right)}}{e^{-\beta U(\mathbf{q})}}\right)
$$

Caso a nova configuração seja aceita, a nova configuração q do sistema é q': $\mathbf{q} \rightarrow \mathbf{q}^{\prime}$

3. Repete os passos 2.1-2.2 até o equilíbrio ser atingido;

4. Calcula todas as propriedades usando a distribuição de posições.

Para provar que esse método gera configurações com probabilidades proporcionais a $e^{-\beta U(\mathbf{q})}$, primeiro deve-se notar que temos duas probabilidades no algoritmo: a probabilidade de transição de uma configuração $\mathbf{q}$ para $\mathbf{q}^{\prime}, \Pi\left(\mathbf{q} \rightarrow \mathbf{q}^{\prime}\right)$, e a probabilidade de aceitação desta transição, $A\left(\mathbf{q} \rightarrow \mathbf{q}^{\prime}\right)$. A primeira está associada ao passo $\mathbf{2 . 1}$, enquanto a segunda ao passo 2.2, [15]. Fica claro que

$$
\Pi\left(\mathbf{q} \rightarrow \mathbf{q}^{\prime}\right)=\Pi\left(\mathbf{q}^{\prime} \rightarrow \mathbf{q}\right)
$$

enquanto que $A\left(\mathbf{q} \rightarrow \mathbf{q}^{\prime}\right) \neq A\left(\mathbf{q}^{\prime} \rightarrow \mathbf{q}\right)$. Para que um sistema se encontre em equilíbro, a probabilidade de encontrar o sistema em uma configuração q deve ser constante no tempo, ou seja,

$$
\frac{\partial P(\mathbf{q})}{\partial t}=0
$$

com a variação temporal de $P(\mathbf{q})$ sendo dada pela equação mestra, [20]:

$$
\frac{\partial P(\mathbf{q})}{\partial t}=\sum_{\mathbf{q}^{\prime}}\left[P\left(\mathbf{q}^{\prime}\right) \Pi\left(\mathbf{q}^{\prime} \rightarrow \mathbf{q}\right) A\left(\mathbf{q}^{\prime} \rightarrow \mathbf{q}\right)-P(\mathbf{q}) \Pi\left(\mathbf{q} \rightarrow \mathbf{q}^{\prime}\right) A\left(\mathbf{q} \rightarrow \mathbf{q}^{\prime}\right)\right]
$$

Usando as equações (3.8) e (3.7) na equação (3.9), temos que

$$
\sum_{\mathbf{q}^{\prime}} \Pi\left(\mathbf{q}^{\prime} \rightarrow \mathbf{q}\right)\left[P\left(\mathbf{q}^{\prime}\right) A\left(\mathbf{q}^{\prime} \rightarrow \mathbf{q}\right)-P(\mathbf{q}) A\left(\mathbf{q} \rightarrow \mathbf{q}^{\prime}\right)\right]=0
$$

Uma condição suficiente para que (3.10) seja satisfeita é a equação de balanço detalhado, 
[20]:

$$
\frac{P\left(\mathbf{q}^{\prime}\right)}{P(\mathbf{q})}=\frac{A\left(\mathbf{q} \rightarrow \mathbf{q}^{\prime}\right)}{A\left(\mathbf{q}^{\prime} \rightarrow \mathbf{q}\right)}, \quad \forall \mathbf{q}, \mathbf{q}^{\prime}
$$

Substituindo $A\left(\mathbf{q} \rightarrow \mathbf{q}^{\prime}\right)$ e $A\left(\mathbf{q}^{\prime} \rightarrow \mathbf{q}\right)$ em (3.11), ficamos com

$$
\frac{P\left(\mathbf{q}^{\prime}\right)}{P(\mathbf{q})}=\frac{\min \left(1, e^{-\beta\left(U\left(\mathbf{q}^{\prime}\right)-U(\mathbf{q})\right)}\right)}{\min \left(1, e^{-\beta\left(U(\mathbf{q})-U\left(\mathbf{q}^{\prime}\right)\right)}\right)}=\frac{e^{-\beta U\left(\mathbf{q}^{\prime}\right)}}{e^{-\beta U(\mathbf{q})}}
$$

Com isso, temos que

$$
P(\mathbf{q}) e^{\beta U(\mathbf{q})}=P\left(\mathbf{q}^{\prime}\right) e^{\beta U\left(\mathbf{q}^{\prime}\right)}=\text { constante }
$$

Como o lado esquerdo de (3.13) só depende de $\mathbf{q}$ e o lado direito de $\mathbf{q}^{\prime}$, devemos ter

$$
P(\mathbf{q}) \propto e^{-\beta U(\mathbf{q})}
$$

Ou seja, com a probabilidade de aceitação do método de Metropolis, temos configurações q com probabilidades proporcionais a $e^{-\beta U(\mathbf{q})}$ sendo geradas. No caso de sistemas discretos o algoritmo aceita transições de microestados $i$ para microestados $j$ com probabilidade $A(i \rightarrow j)=\min \left(1, e^{-\beta\left(E_{j}-E_{i}\right)}\right)$. Com isso, são gerados microestados $i$ com probabilidades proporcionais a $e^{-\beta E_{i}}$.

\subsection{Método de Ray}

O método de Monte Carlo de Metropolis é aplicado para sistemas em equilíbrio térmico com um reservatório (ensemble canônico). Um método de Monte Carlo microcanônico foi apresentado por Micheal Creutz em 1983, [8]. O método é formulado para sistemas sem energia cinética e introduz um demônio, com energia $E_{D}$, que interage com o sistema, de tal forma que a energia total, dada por $E=U(\mathbf{q})+E_{D}$, é conservada. Em 1991, John R. Ray apresenta um método de Monte Carlo microcanônico, seguindo os mesmos princípios do método de Metropolis, que pode ser aplicado em sistemas com energia cinética, [18]. Seguindo o trabalho de Pearson et al., [17], a média de uma grandeza $X$ em um sistema isolado (ensemble microcanônico) composto de $N$ partículas, volume $V$ e energia $E$ é dada por: 


$$
<X>=\frac{\epsilon_{0}}{h^{d N} N !} \int X(\mathbf{p}, \mathbf{q}) \frac{\delta\left(E-H_{N}(\mathbf{p}, \mathbf{q})\right)}{W_{N}(E, V)} d \mathbf{p} d \mathbf{q}=\int X(\mathbf{p}, \mathbf{q}) P(\mathbf{p}, \mathbf{q}) d \mathbf{p} d \mathbf{q}
$$

com $H_{N}(\mathbf{q}, \mathbf{p})$ sendo a hamiltoniana de um sistema composto de $N$ partículas, $h$ a constante de Plank, $\epsilon_{0}$ uma constante qualquer com unidade de energia e $d$ a dimensão do sistema. A função de partição microcanônica pode ser escrita como

$$
W_{N}(E, V)=\frac{\epsilon_{0}}{h^{d N} N !} \int \delta\left(E-H_{N}(\mathbf{p}, \mathbf{q})\right) d \mathbf{p} d \mathbf{q}=\int \omega(\mathbf{p}, \mathbf{q}) d \mathbf{p} d \mathbf{q}
$$

com $\omega(\mathbf{p}, \mathbf{q})$ sendo a densidade de probabilidade no espaço de fase. Assim como no método de Metropolis, devemos ter uma densidade de probabilidade que dependa apenas de $\mathbf{q}$. Aplicando uma transformada de Laplace em (3.16), ficamos com

$$
\begin{aligned}
Z_{N}(\beta, V) & =\int_{0}^{\infty} e^{-\beta E} W_{N}(E, V) d E= \\
& =\frac{\epsilon_{0}}{h^{d N} N !} \int_{0}^{\infty} \int d \mathbf{p} d \mathbf{q} d E e^{-\beta E} \delta\left(E-H_{N}(\mathbf{p}, \mathbf{q})\right)= \\
& =\frac{\epsilon_{0}}{h^{d N} N !} \int d \mathbf{p} d \mathbf{q} e^{-\beta[K(\mathbf{p})+U(\mathbf{q})]},
\end{aligned}
$$

onde $K(\mathbf{p})$ e $U(\mathbf{q})$ são as energias cinética e potencial, respectivamente. Seja a parte cinética dada por $K(\mathbf{p})=\sum_{i} \frac{\mathbf{p}_{i}^{2}}{2 m}$, temos

$$
\begin{aligned}
Z_{N}(\beta, V) & =\frac{\epsilon_{0}}{h^{d N} N !} \int d \mathbf{q} \exp [-\beta U(\mathbf{q})] \int d \mathbf{p} \exp \left(-\beta \sum_{i=1}^{N} \frac{p_{i}^{2}}{2 m}\right)= \\
& =\frac{\epsilon_{0}}{h^{d N} N !} \int d \mathbf{q} \exp [-\beta U(\mathbf{q})] \int d \mathbf{p} \prod_{i=1}^{N} \exp \left(-\beta \frac{p_{i}^{2}}{2 m}\right)= \\
& =\frac{\epsilon_{0}}{h^{d N} N !} \int d \mathbf{q} \exp [-\beta U(\mathbf{q})]\left[\int_{-\infty}^{\infty} d p \exp \left(-\beta \frac{p^{2}}{2 m}\right)\right]^{d N}
\end{aligned}
$$

Usando $\int_{-\infty}^{\infty} e^{-\alpha x^{2}} d x=\sqrt{\frac{\pi}{\alpha}}$ em (3.18), chegamos em

$$
Z_{N}(\beta, V)=\frac{\epsilon_{0}}{h^{d N} N !}\left(\frac{2 m \pi}{\beta}\right)^{\frac{d N}{2}} \int d \mathbf{q} e^{-\beta U(\mathbf{q})} .
$$

Aplicando a transformada inversa de Laplace em (3.19), temos que $W_{N}(E, V)$ fica 


$$
W_{N}(E, V)=\mathcal{L}^{-1}\left\{Z_{N}(\beta, V)\right\}=\frac{\epsilon_{0}}{h^{d N} N !} \int d \mathbf{q} \mathcal{L}^{-1}\left\{\left(\frac{2 m \pi}{\beta}\right)^{\frac{d N}{2}} e^{-\beta U(\mathbf{q})}\right\}
$$

Usando $\mathcal{L}^{-1}\left\{\frac{\Gamma(n+1) e^{-s \tau}}{(s+a)^{n+1}}\right\}=(t-\tau)^{n} e^{-a(t-\tau)} \Theta(t-\tau)$ em $(3.20)$, ficamos com

$$
\begin{gathered}
W_{N}(E, V)=\frac{\epsilon_{0}}{\Gamma\left(\frac{d N}{2}\right) N !}\left(\frac{2 m \pi}{h^{2}}\right)^{\frac{d N}{2}} \int d \mathbf{q}(E-U(\mathbf{q}))^{\frac{d N}{2}-1} \Theta(E-U(\mathbf{q})) . \\
\therefore \omega(\mathbf{q})=\frac{\epsilon_{0}}{\Gamma\left(\frac{d N}{2}\right) N !}\left(\frac{2 m \pi}{h^{2}}\right)^{\frac{d N}{2}}(E-U(\mathbf{q}))^{\frac{d N}{2}-1} \Theta(E-U(\mathbf{q})) .
\end{gathered}
$$

Das equações (1.40) e (3.21), temos que

$$
S_{N}(E, V)=\ln W_{N}(E, V)=\ln \left[\int d \mathbf{q}(E-U(\mathbf{q}))^{\frac{d N}{2}-1} \Theta(E-U(\mathbf{q}))\right]+S_{N 0},
$$

$\operatorname{com} S_{N 0}=\ln \left[\frac{\epsilon_{0}}{\Gamma\left(\frac{d N}{2}\right) N !}\left(\frac{2 m \pi}{h^{2}}\right)^{\frac{d N}{2}}\right]$. Derivando (3.23) em relação a $E$ e usando temos que a temperatura é dada por

$$
\begin{aligned}
& \frac{1}{T}=\frac{\partial S}{\partial E}=\frac{\int d \mathbf{q}\left(\frac{d N}{2}-1\right) \frac{(E-U(\mathbf{q}))^{\frac{d N}{2}-1}}{(E-U(\mathbf{q}))} \Theta(E-U(\mathbf{q}))}{\int d \mathbf{q}(E-U(\mathbf{q}))^{\frac{d N}{2}-1} \Theta(E-U(\mathbf{q}))}+ \\
& +\frac{\int d \mathbf{q}(E-U(\mathbf{q}))^{\frac{d N}{2}-1} \delta(E-U(\mathbf{q}))}{\int d \mathbf{q}(E-U(\mathbf{q}))^{\frac{d N}{2}-1} \Theta(E-U(\mathbf{q}))}= \\
& =\frac{\left(\frac{d N}{2}-1\right) \int d \mathbf{q}(E-U(\mathbf{q}))^{-1}(E-U(\mathbf{q}))^{\frac{d N}{2}-1} \Theta(E-U(\mathbf{q}))}{\int d \mathbf{q}(E-U(\mathbf{q}))^{\frac{d N}{2}-1} \Theta(E-U(\mathbf{q}))}= \\
& =\frac{\left(\frac{d N}{2}-1\right) \int d \mathbf{q}(E-U(\mathbf{q}))^{-1} \omega(\mathbf{q})}{W_{N}(E, V)}=\left(\frac{d N}{2}-1\right) \int d \mathbf{q}(E-U(\mathbf{q}))^{-1} P(\mathbf{q})= \\
& =\left(\frac{d N}{2}-1\right) \frac{1}{<E-U(\mathbf{q})>}=\left(\frac{d N}{2}-1\right) \frac{1}{<K>}
\end{aligned}
$$

$$
\therefore T=\frac{2}{d N-2}<K>
$$


A densidade de probabilidade em um sistema isolado fica dada por

$$
P(\mathbf{q})=\frac{\omega(\mathbf{q})}{W_{N}(E, V)}=\frac{(E-U(\mathbf{q}))^{\frac{d N}{2}-1} \Theta(E-U(\mathbf{q}))}{\int d \mathbf{q}(E-U(\mathbf{q}))^{\frac{d N}{2}-1} \Theta(E-U(\mathbf{q}))} .
$$

Portanto, seguindo o mesmo modelo do método de Metropolis, o algoritmo de Ray segue como:

1. Define uma energia $E$ e uma condição inicial para o sistema, q, com energia potencial $U(\mathbf{q})<E$

2.1. Muda aleatoriamente a posição de uma partícula, $\mathbf{q} \rightarrow \mathbf{q}^{\prime}$, e computa a nova energia potencial $U\left(\mathbf{q}^{\prime}\right)$;

2.2. Aceita a nova configuração com a probabilidade:

$$
A\left(\mathbf{q} \rightarrow \mathbf{q}^{\prime}\right)=\min \left(1, \frac{\left(E-U\left(\mathbf{q}^{\prime}\right)\right)^{\frac{d N}{2}-1} \Theta\left(E-U\left(\mathbf{q}^{\prime}\right)\right)}{(E-U(\mathbf{q}))^{\frac{d N}{2}-1}}\right)
$$

Caso a nova configuração seja aceita, a nova configuração q do sistema é q': $\mathbf{q} \rightarrow \mathbf{q}^{\prime}$

3. Repete os passos 2.1-2.2 até o equilíbrio ser atingido;

4. Calcula todas as propriedades usando a distribuição de posições.

Desta forma, são gerados pontos q com probabilidades proporcionais a $(E-U(\mathbf{q}))^{\frac{d N}{2}-1}$, de onde é possível calcular todas as médias de grandezas macroscópicas relacionadas a um sistema isolado.

\subsection{Método de Wang-Landau}

Em 2001, Fugao Wang e David P. Landau apresentaram um método de Monte Carlo para calcular a densidade de estados $W$ de um sistema, [25]. Diferente do método de Metropolis, que gera microestados $i$ seguindo uma distribuição canônica para uma dada temperatura $e^{-\beta E_{i}}$, o método de Wang-Landau tem como objetivo estimar a densidade de estados $W(E)$ através de um passeio aleatório em todos os microestados possíveis do sistema. A probabilidade de encontrar o sistema em um microestado $i$ com energia $E_{i}$ é dada por

$$
P_{i}=\frac{1}{W\left(E_{i}\right)}
$$


Como a densidade de estados do sistema não é conhecida, usa-se uma densidade de estados estimada $g(E)$ que é modificada constantemente para que a condição de balanço detalhado seja satisfeita. Neste caso, a probabilidade de aceitação, é dada por

$$
A(i \rightarrow j)=\min \left(1, \frac{\frac{1}{g\left(E_{j}\right)}}{\frac{1}{g\left(E_{i}\right)}}\right)=\min \left(1, \frac{g\left(E_{i}\right)}{g\left(E_{j}\right)}\right) .
$$

Para aproximar $g(E)$ do valor real $W(E)$, o algoritmo de Wang-Landau trabalha da seguinte maneira:

1. Estabelece $g(E)=1$ e o histograma $H(E)=0, \forall E$. Define o fator de modificação $f_{0}=e^{1}$ e um estado inicial $i$, com energia $E_{i}$, para o sistema;

2.1. Muda aleatoriamente o estado de uma partícula, $i \rightarrow j$, e computa a nova energia $E_{j}$;

2.2. Aceita o novo estado com a probabilidade:

$$
A(i \rightarrow j)=\min \left(1, \frac{g\left(E_{i}\right)}{g\left(E_{j}\right)}\right)
$$

Caso o novo estado seja aceito, o novo estado $i$ do sistema é $j: i \rightarrow j$;

2.3. Modifica $g\left(E_{i}\right) \rightarrow g\left(E_{i}\right) f$ e $H\left(E_{i}\right) \rightarrow H\left(E_{i}\right)+1$;

2.4. Se o histograma $H(E)$ não está "plano", retorna ao passo $\mathbf{2 . 1}$;

2.5. Se o histograma $H(E)$ está "plano", modifica $f=f^{\frac{1}{2}}$ e coloca $H(E)=0, \forall$ $E$

3. Repete os passos 2.1-2.5 até que $f=f_{\text {min }}$;

4. Calcula as propriedades usando a densidade de estados final $g(E)$.

Para mostrar que o algoritmo aproxima $g(E)$ de $W(E)$, primeiro deve-se notar que, no início, a condição de balanço detalhado (3.11) não é satisfeita

$$
\frac{P_{j}}{P_{i}}=\frac{W\left(E_{i}\right)}{W\left(E_{j}\right)} \neq \frac{g\left(E_{i}\right)}{g\left(E_{j}\right)}=\frac{A(i \rightarrow j)}{A(j \rightarrow i)} .
$$

Com (3.27) e (3.28), a equação (3.9) fica escrita como

$$
\begin{aligned}
\frac{\partial P_{i}}{\partial t} & =\sum_{j}\left[\Pi(j \rightarrow i) A(j \rightarrow i) P_{i}\left(\frac{P_{j}}{P_{i}}-\frac{A(i \rightarrow j)}{A(j \rightarrow i)}\right)\right]= \\
& =\sum_{j}\left[\Pi(j \rightarrow i) A(j \rightarrow i) P_{i}\left(\frac{W\left(E_{i}\right)}{W\left(E_{j}\right)}-\frac{g\left(E_{i}\right)}{g\left(E_{j}\right)}\right)\right] .
\end{aligned}
$$


Como $\Pi(j \rightarrow i) A(j \rightarrow i) P_{i}>0$, sejam dois estados, $i$ e $j$, quaisquer, se $\frac{W\left(E_{i}\right)}{W\left(E_{j}\right)}>\frac{g\left(E_{i}\right)}{g\left(E_{j}\right)}$, então esse termo contribui para que $\frac{\partial P_{i}}{\partial t}>0$, ou seja, o estado $i$ é visitado mais vezes em relação ao estado $j$, aumentando $g\left(E_{i}\right)$. Entretanto, se $\frac{W\left(E_{i}\right)}{W\left(E_{j}\right)}<\frac{g\left(E_{i}\right)}{g\left(E_{j}\right)}$, então esse termo contribui para que $\frac{\partial P_{i}}{\partial t}<0$, ou seja, o estado $j$ é visitado mais vezes em relação ao estado $i$, aumentando $g\left(E_{j}\right)$. Desta forma, a tendência é de que a condição de balanço detalhado seja satisfeita:

$$
\frac{W\left(E_{i}\right)}{W\left(E_{j}\right)} \simeq \frac{g\left(E_{i}\right)}{g\left(E_{j}\right)}, \quad \forall i, j .
$$

A precisão do método de Wang-Landau, o quanto $g(E)$ se aproxima da densidade de estados real, é da ordem de $\ln f$, [25], onde $f$ é o fator de modificação. Porém, um fator de modificação muito próximo de 1 aumenta o tempo de convergência de $g(E)$. Portanto, o ideal é começar com um valor de $f$ grande, $f_{0}=e^{1} \simeq 2.718281$, e diminuir gradativamente até um valor próximo de $1, f_{\min }=1.000001$. Além da forma usual de diminuir o fato de modificação (passo 2.5), outras formas mais eficientes foram propostas, [4, 7]. O critério para diminuir o fator de modificação é que o histograma $H(E)$ seja "plano"(como ele nunca será perfeitamente plano, a condição real é que $H(E)$ não seja menor do que, por exemplo, $70 \%$ do valor médio do histograma $\langle H(E)>, \forall E$ ), desta forma, é garantido que o sistema terá visitado todos os valores possíveis de $E$. Uma vez obtida a densidade de estados $g(E)$, a entropia é encontrada através da equação (1.40)

$$
S(E)=\ln g(E)
$$

O método de Wang-Landau, proposto originalmente, é aplicado apenas para sistemas discretos. A generalização para sistemas contínuos veio em 2002 com Shell et al., [22]. A ideia é escrever a função de partição microcanônica sem as integrais nos momentos, dependendo apenas de uma densidade de estados configuracional. Partindo da expressão (1.51), a função de partição microcanônica pode ser escrita como

$$
\begin{aligned}
W_{N}(E, V) & =\frac{\epsilon_{0}}{h^{d N} N !} \int \delta\left(E-H_{N}(\mathbf{q}, \mathbf{p})\right) d \mathbf{q} d \mathbf{p}= \\
& =\frac{\epsilon_{0}}{h^{d N} N !} \int \delta(E-K(\mathbf{p})-U(\mathbf{q})) d \mathbf{q} d \mathbf{p}
\end{aligned}
$$


Usando a propriedade $\int_{-\infty}^{\infty} \delta(\xi-x) \delta(x-\eta) d x=\delta(\xi-\eta)$ da função delta em (3.32),

$$
\begin{aligned}
W_{N}(E, V) & =\frac{\epsilon_{0}}{h^{d N} N !} \int d \mathbf{q} d \mathbf{p} \delta(E-K(\mathbf{p})-U(\mathbf{q}))= \\
& =\frac{\epsilon_{0}}{h^{d N} N !} \int_{-\infty}^{\infty} d \varepsilon \int d \mathbf{q} d \mathbf{p} \delta(E-K(\mathbf{p})-\varepsilon) \delta(\varepsilon-U(\mathbf{q}))= \\
& =\frac{\epsilon_{0}}{h^{d N} N !} \int_{-\infty}^{\infty}\left[\int \delta(E-K(\mathbf{p})-\varepsilon) d \mathbf{p}\right]\left[\int \delta(\varepsilon-U(\mathbf{q})) d \mathbf{q}\right] d \varepsilon= \\
& =\int_{-\infty}^{\infty} W_{K}(E-\varepsilon, V) W_{U}(\varepsilon, V) d \varepsilon
\end{aligned}
$$

com

$$
\begin{gathered}
W_{K}(E-\varepsilon, V)=\frac{\epsilon_{0}}{h^{d N} N !} \int \delta(E-K(\mathbf{p})-\varepsilon) d \mathbf{p}, \\
W_{U}(\varepsilon, V)=\int \delta(\varepsilon-U(\mathbf{q})) d \mathbf{q} .
\end{gathered}
$$

De (3.35), fica claro que $W_{U}(\varepsilon, V)$ é uma densidade de estados configuracional, com $\varepsilon$ sendo a energia potencial. Se a parte cinética do hamiltoniano é dada por $K(\mathbf{p})=\sum_{i} \frac{\mathbf{p}_{i}^{2}}{2 m}$, então podemos resolver $W_{K}(E-\varepsilon, V)$ aplicando uma transformada de Laplace em (3.34),

$$
\begin{aligned}
Z_{K}(\beta, V) & =\int_{0}^{\infty} e^{-\beta(E-\varepsilon)} W_{K}(E-\varepsilon, V) d(E-\varepsilon)= \\
& =\frac{\epsilon_{0}}{h^{d N} N !} \int_{0}^{\infty} \int e^{-\beta(E-\varepsilon)} \delta(E-K(\mathbf{p})-\varepsilon) d \mathbf{p} d(E-\varepsilon)= \\
& =\frac{\epsilon_{0}}{h^{d N} N !} \int e^{-\beta K(\mathbf{p})} d \mathbf{p}=\frac{\epsilon_{0}}{h^{d N} N !} \int \exp \left(-\beta \sum_{i=1}^{N} \frac{p_{i}^{2}}{2 m}\right) d \mathbf{p}= \\
& =\frac{\epsilon_{0}}{h^{d N} N !} \int \prod_{i=1}^{N} \exp \left(-\beta \frac{p_{i}^{2}}{2 m}\right) d \mathbf{p}= \\
& =\frac{\epsilon_{0}}{h^{d N} N !}\left[\int_{-\infty}^{\infty} \exp \left(-\beta \frac{p^{2}}{2 m}\right) d p\right]^{d N} .
\end{aligned}
$$

Usando $\int_{-\infty}^{\infty} e^{-\alpha x^{2}} d x=\sqrt{\frac{\pi}{\alpha}}$ em (3.36), temos

$$
Z_{K}(\beta, V)=\frac{\epsilon_{0}}{h^{d N} N !}\left(\frac{2 m \pi}{\beta}\right)^{\frac{d N}{2}} .
$$

Usando a transformada inversa de Laplace $\mathcal{L}^{-1}\left\{\frac{\Gamma(n+1)}{s^{n+1}}\right\}=t^{n} \Theta(t)$ em (3.37) obtemos 
$W_{K}(E-\varepsilon, V):$

$$
\begin{aligned}
W_{K}(E-\varepsilon, V) & =\mathcal{L}^{-1}\left\{Z_{K}(\beta, V)\right\}=\frac{\epsilon_{0}}{h^{d N} N !} \mathcal{L}^{-1}\left\{\left(\frac{2 m \pi}{\beta}\right)^{\frac{d N}{2}}\right\}= \\
& =\frac{\epsilon_{0}}{\Gamma\left(\frac{d N}{2}\right) N !}\left(\frac{2 m \pi}{h^{2}}\right)^{\frac{d N}{2}} \mathcal{L}^{-1}\left\{\frac{\Gamma\left(\frac{d N}{2}\right)}{\beta^{\frac{d N}{2}}}\right\}= \\
& =\frac{\epsilon_{0}}{\Gamma\left(\frac{d N}{2}\right) N !}\left(\frac{2 m \pi}{h^{2}}\right)^{\frac{d N}{2}}(E-\varepsilon)^{\frac{d N}{2}-1} \Theta(E-\varepsilon) .
\end{aligned}
$$

Substituindo (3.38) em (3.33), a função de partição microcanônica fica expressa como

$$
W_{N}(E, V)=\frac{\epsilon_{0}}{\Gamma\left(\frac{d N}{2}\right) N !}\left(\frac{2 m \pi}{h^{2}}\right)^{\frac{d N}{2}} \int_{-\infty}^{\infty}(E-\varepsilon)^{\frac{d N}{2}-1} \Theta(E-\varepsilon) W_{U}(\varepsilon, V) d \varepsilon
$$

Para obter $W_{U}(\varepsilon, V)$, pode-se utilizar o método de Wang-Landau para sistemas contínuos, [22], que é executado da seguinte forma:

1. Estabelece $g(U)=1$ e o histograma $H(U)=0, \forall U$. Define o fator de modificação $f_{0}=e^{1}$ e uma configuração inicial $\mathbf{q}$, com energia potencial $U(\mathbf{q})$, para o sistema;

2.1. Muda aleatoriamente a posição de uma partícula, $\mathbf{q} \rightarrow \mathbf{q}^{\prime}$, e computa a nova energia potencial $U\left(\mathbf{q}^{\prime}\right)$;

2.2. Aceita a nova configuração com a probabilidade:

$$
A\left(\mathbf{q} \rightarrow \mathbf{q}^{\prime}\right)=\min \left(1, \frac{g(U(\mathbf{q}))}{g\left(U\left(\mathbf{q}^{\prime}\right)\right)}\right)
$$

Caso a nova configuração seja aceita, a nova configuração q do sistema é q': $\mathbf{q} \rightarrow \mathbf{q}^{\prime}$

2.3. Modifica $g(U(\mathbf{q})) \rightarrow g(U(\mathbf{q})) f$ e $H(U(\mathbf{q})) \rightarrow H(U(\mathbf{q}))+1$;

2.4. Se a condição de mínimo de $H(U)$ não é satisfeita, retorna ao passo 2.1;

2.5. Se a condição de mínimo de $H(U)$ é satisfeita, modifica $f=f^{\frac{1}{2}}$ e coloca $H(U)=0, \forall U$

3. Repete os passos 2.1-2.5 até que $f=f_{\text {min }}$;

4. Calcula as propriedades usando a densidade de estados final $g(U)$. 
Com isso, $g(U) \rightarrow W_{U}(U)$. Substituindo $g(U)$ em (3.39) temos a função de partição microcanônica e, da equação (1.40), a entropia do sistema

$$
S_{N}(E)=\ln W_{N}(E)=\ln \left[\int_{-\infty}^{\infty}(E-U)^{\frac{d N}{2}-1} \Theta(E-U) g(U) d U\right]+S_{N 0},
$$

$\operatorname{com} S_{N 0}=\ln \left[\frac{\epsilon_{0}}{\Gamma\left(\frac{d N}{2}\right) N !}\left(\frac{2 m \pi}{h^{2}}\right)^{\frac{d N}{2}}\right]$. Uma vez obtida a entropia, toda a termodinâmica do sistema é derivada dela. É importante notar a diferença nos passos 2.4 e $\mathbf{2 . 5}$ (proposto por Shell et al., [22]), onde o critério para diminuir o fator de modificação é que a energia menos visitada do histograma $H(E)$ tenha sido visitada um número mínimo de vezes (e.g., 20 vezes). Essa condição é mais fraca do que a condição do histograma ser "plano", mas ainda garante que o sistema visite todas as energia possíveis. 


\section{Capítulo 4}

\section{Resultados}

No Capítulo 3 são apresentados alguns métodos de Monte Carlo microcanônicos, o método de Ray e o de Wang-Landau. Neste capítulo são apresentadas soluções analíticas de equilíbrio de alguns modelos de sistemas com interações de longo alcance, que são mostrados como exemplos no Capítulo 2, junto de resultados numéricos obtidos utilizando os métodos de Monte Carlo microcanônicos do Capítulo 3. Também é realizada uma análise sobre a aplicabilidade dos métodos e, quando igualmente aplicados, é feita uma comparação de desempenho, com o intuito de descobrir qual método é recomendado em diferentes situações. Os programas utilizados nos cálculos numéricos apresentados neste capítulo foram escritos na linguagem de programação $C$, os códigos fonte dos programas principais encontram-se no Apêndice A.

\subsection{Curie-Weiss}

O modelo de Curie-Weiss é apresentado na seção 2.2 como um exemplo de sistema com energia extensiva e não-aditiva. A hamiltoniana de Curie-Weiss é dada por

$$
H=-\frac{J}{2 N}\left(\sum_{i=1}^{N} S_{i}\right)^{2}
$$

onde os spins podem assumir os valores $S_{i}= \pm 1$ e $J$ é uma constante de acoplamento, quando $J>0$ temos o caso ferromagnético e quando $J<0$ o caso antiferromagnético. Em termos da magnetização intensiva do sistema, equação (2.9), a hamiltoniana é expressa como

$$
H=-\frac{J N}{2} m^{2}
$$


Para obter a entropia do sistema, devemos calcular o número de microestados $W$ para uma dada energia $E$. Sendo $N_{+}$e $N_{-}$o número de spins +1 e -1 , respectivamente, temos

$$
W=\frac{N !}{N_{+} ! N_{-} !} .
$$

O número de partículas do sistema é $N=N_{+}+N_{-}$, e a magnetização extensiva é $M=$ $N_{+}-N_{-}$(pela expressão $\left.(2.9)\right)$, portanto

$$
\begin{gathered}
N_{+}=\frac{1}{2}(N+M) \quad \text { e } N_{-}=\frac{1}{2}(N-M), \\
\therefore W=\frac{N !}{\left[\frac{(N+M)}{2}\right] !\left[\frac{(N-M)}{2}\right] !}
\end{gathered}
$$

com isso,

$$
\ln W=\ln N !-\ln \left[\frac{N+M}{2}\right] !-\ln \left[\frac{N-M}{2}\right] !
$$

Usando a aproximação de Stirling em (4.6),

$$
\begin{aligned}
\ln W & \simeq N \ln N-N-\left(\frac{N+M}{2}\right) \ln \left(\frac{N+M}{2}\right)- \\
& -\left(\frac{N-M}{2}\right) \ln \left(\frac{N-M}{2}\right)+\frac{N+M}{2}+\frac{N-M}{2}= \\
& =N \ln N-\left(\frac{N+M}{2}\right) \ln \left(\frac{N+M}{2}\right)-\left(\frac{N-M}{2}\right) \ln \left(\frac{N-M}{2}\right) \\
& \therefore \ln W \simeq N \ln N-\frac{N}{2} \ln \left(\frac{N^{2}-M^{2}}{4}\right)+\frac{M}{2} \ln \left(\frac{N-M}{N+M}\right) .
\end{aligned}
$$

Substituindo a magnetização extensiva pela magnetização intensiva, $M=N m$, em (4.7),

$$
\begin{aligned}
\ln W= & N \ln N-\frac{N}{2} \ln \left[N^{2}\left(\frac{1-m^{2}}{4}\right)\right]+\frac{N m}{2} \ln \left(\frac{1-m}{1+m}\right)= \\
= & N \ln N-\frac{N}{2} \ln N^{2}-\frac{N}{2} \ln \left(\frac{1-m^{2}}{4}\right)+\frac{N m}{2} \ln \left(\frac{1-m}{1+m}\right) \\
& \therefore \ln W=\frac{N}{2}\left[m \ln \left(\frac{1-m}{1+m}\right)-\ln \left(\frac{1-m^{2}}{4}\right)\right]
\end{aligned}
$$


Pela equação (4.2), a densidade de energia é dada por

$$
e=\frac{E}{N}=-\frac{J m^{2}}{2}
$$

Substituindo (4.9) em (4.8), a entropia por partícula $s(e)=\frac{S_{N}(E)}{N}$ é expressa como

$$
s_{ \pm}(e)=\frac{1}{2}\left[\sqrt{\mp 2 e} \ln \left(\frac{1-\sqrt{\mp 2 e}}{1+\sqrt{\mp 2 e}}\right)-\ln \left(\frac{1 \pm 2 e}{4}\right)\right],
$$

onde é fixado $|J|=1$ por praticidade, $s_{+}(e)$ e $s_{-}(e)$ são as entropias para o caso ferromagnético $(J=+1)$ e antiferromagnético $(J=-1)$, respectivamente.

Como o modelo de Curie-Weiss é discreto, para obter a entropia numericamente, deve ser utilizado o método de Wang-Landau original (que é aplicado em sistemas discretos). Como apresentado no Capítulo 3, a proposta de F. Wang e D. P. Landau é diminuir o fator de modificação, $f$, sempre que o histograma de energia for "plano", [25]. Enquanto que a proposta de Shell et al., [22], é de que o fator de modificação seja alterado sempre que a energia menos visitada tenha sido visitada um número mínimo de vezes. Entretanto, diferente do método de Ray, onde o tempo de execução do programa é definido manualmente através do número de passos, o método de Wang-Landau possui critérios de parada que podem ser satisfeitos rapidamente ou apenas em tempos muito longos, fazendo com que mesmas condições iniciais tenham tempos de execução muito distintos. Como o objetivo é comparar o método de Wang-Landau com o de Ray, ambos devem ter um critério de parada manual. A proposta deste trabalho é que o fator de modificação seja alterado sempre que um número pré-determinado de passos seja cumprido. É recomendado um número de passos grande o suficiente para que todas as energias sejam visitadas para valores grande de $f$. Com esse critério, o tempo de execução de um programa é praticamente o mesmo para as mesmas condições iniciais. A figura 4.1 mostra as entropias teóricas do modelo de Curie-Weiss, (4.10), junto das entropias obtidas numericamente, para $10^{3}$ partículas, utilizando diferentes critérios para alterar o fator de modificação. Na figura 4.2 estão os erros relativos das entropias $\delta S_{x}$, dados pela fórmula

$$
\delta S_{x}=\left|\frac{s(e)-s_{x}(e)}{s(e)}\right|,
$$

onde $s(e)$ é a entropia teórica e $x$ indica o critério utilizado no cálculo numérico, podendo ser plano (para o critério original de F. Wang e D. P. Landau), minimo (para o critério proposto por Shell et al.) e passos (para o critério proposto neste trabalho). 


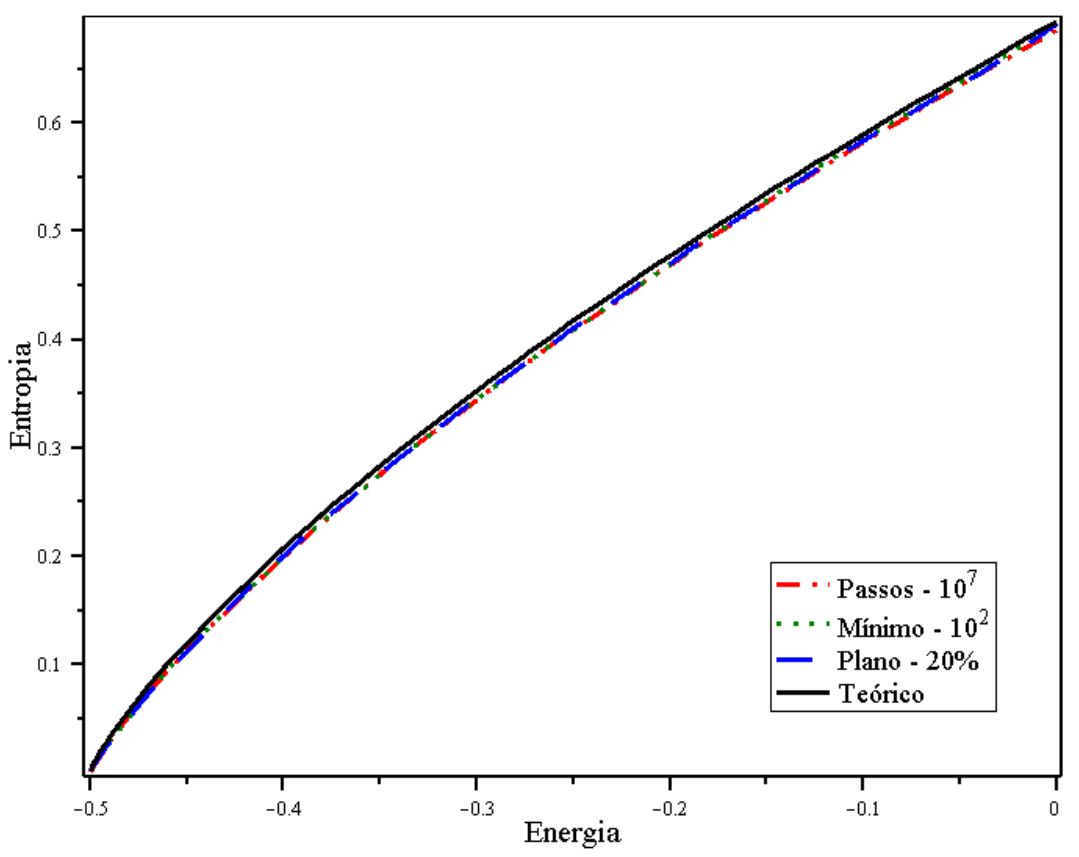

(a) Entropia (ferromagnético)

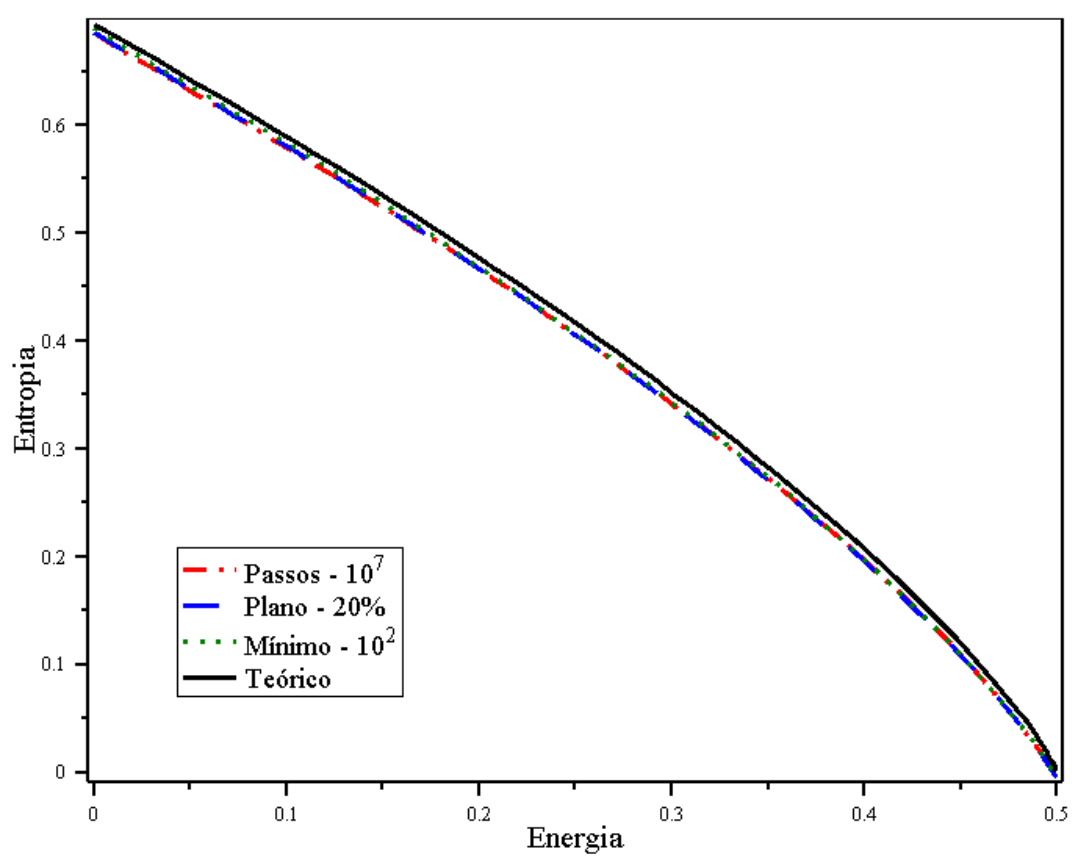

(b) Entropia (antiferromagnético)

Figura 4.1: (a) Entropia vs. Energia (ferromagnético). (b) Entropia vs. Energia (antiferromagnético) para o modelo de Curie-Weiss. 


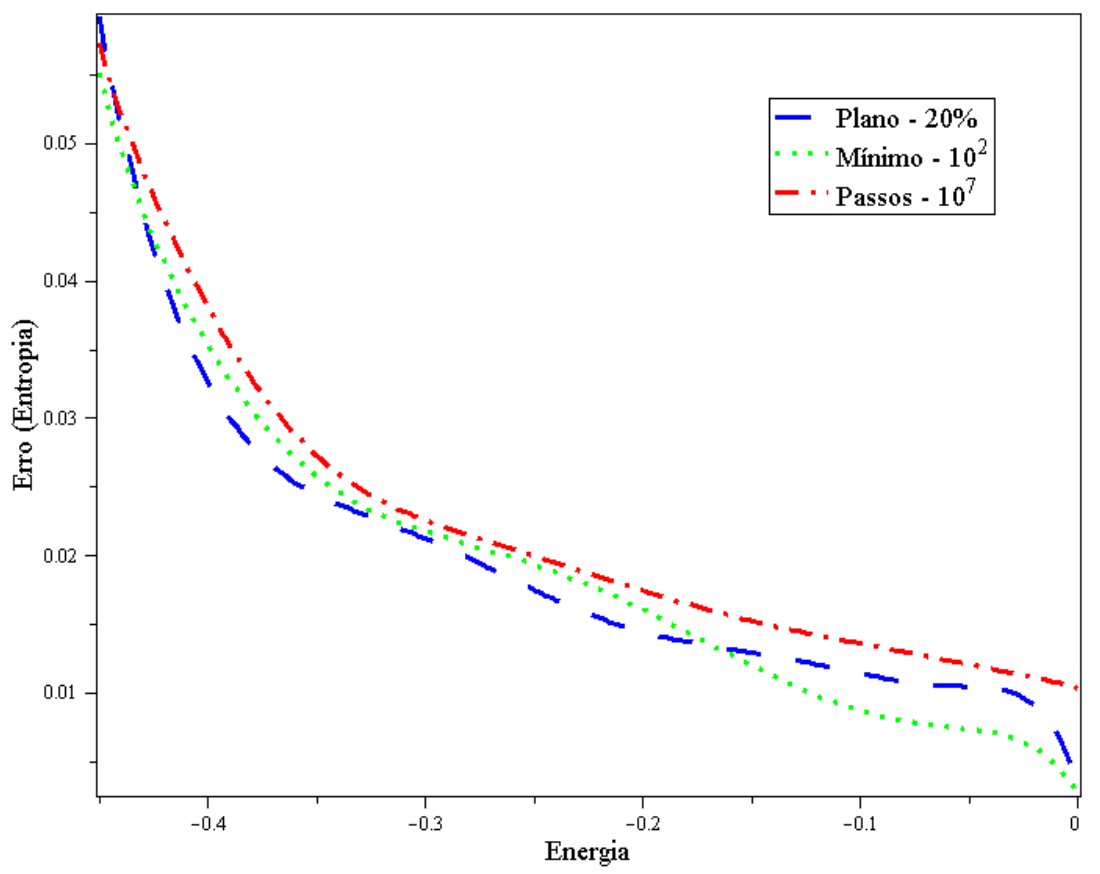

(a) Erro Entropia (ferromagnético)

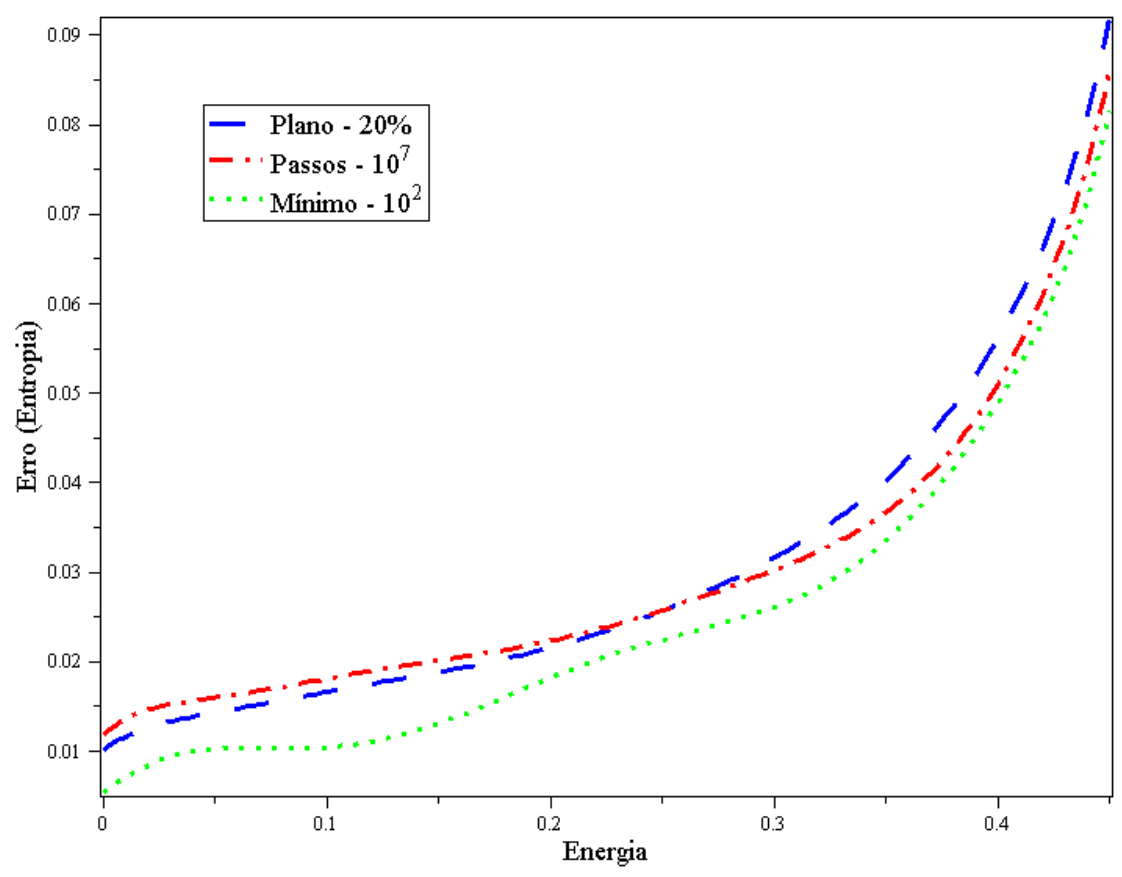

(b) Erro Entropia (antiferromagnético)

Figura 4.2: (a) Erro da entropia vs. Energia (ferromagnético). (b) Erro da entropia vs. Energia (antiferromagnético) para o modelo de Curie-Weiss. 
Nota-se que todos os critérios de modificação apresentam resultados com erros relativos da ordem de $10^{-1}$. Sendo o critério do número de passos com erros maiores, em média. Contudo, o valor absoluto da entropia não é importante no contexto da termodinâmica, uma vez que toda a informação do sistema é obtida através de suas derivadas. A figura 4.3 mostra as temperaturas obtidas a partir da derivação das entropias numéricas, junto da temperatura teórica. Os erros relativos de cada temperatura $\delta T_{x}$, estão na figura 4.4 e são obtidos pela fórmula

$$
\delta T_{x}=\left|\frac{T(e)-T_{x}(e)}{T(e)}\right|
$$

onde $T(e)$ é a entropia teórica e $x$ indica o critério utilizado no cálculo numérico, podendo ser plano, minimo e passos. Os erros para a temperatura ficam em torno de $10^{-1}$. Neste caso, o critério do número de passos apresenta os menores erros, em média. A diferença de precisão entre os critérios de modificação é muito pequena, porém o tempo de execução do programa varia bastante. A tabela 4.1 mostra os valores médios dos tempos de execução de cada um dos critérios utilizados mantendo a precisão entre eles.

\begin{tabular}{|c|c|c|c|c|c|c|}
\hline \multirow{2}{*}{$\begin{array}{c}\text { Número de } \\
\text { Partículas }\end{array}$} & \multicolumn{2}{|c|}{ Passos } & \multicolumn{2}{c|}{ Mínimo } & \multicolumn{2}{c|}{ Plano } \\
\cline { 2 - 7 } & Tempo (s) & $\begin{array}{c}\text { Número } \\
\text { de Passos }\end{array}$ & Tempo (s) & $\begin{array}{c}\text { Visitação } \\
\text { Mínima }\end{array}$ & Tempo (s) & $\begin{array}{c}\% \\
\text { Mínima }\end{array}$ \\
\hline 10 & 0.21 & $10^{5}$ & $\sim 0.001$ & 100 & $\sim 0.001$ & $20 \%$ \\
\hline 100 & 4.53 & $10^{6}$ & 0.17 & 100 & 0.35 & $20 \%$ \\
\hline 1000 & 282.29 & $10^{7}$ & 219.22 & 100 & 270.71 & $20 \%$ \\
\hline
\end{tabular}

Tabela 4.1: Tempos médios de execução para diferentes critérios de modificação do método de Wang-Landau aplicado ao modelo de Curie-Weiss.

Na figura 4.5 estão os valores da tabela 4.1. Apesar dos critérios plano e mínimo serem mais rápidos do que o critério passos, pelo comportamento do tempo de execução em função no número de partículas, é de se esperar que para um número grande de partículas o critério de modificação passos seja vantajoso. O critério de modificação baseado no número de passos será o critério utilizado daqui para frente neste trabalho. 


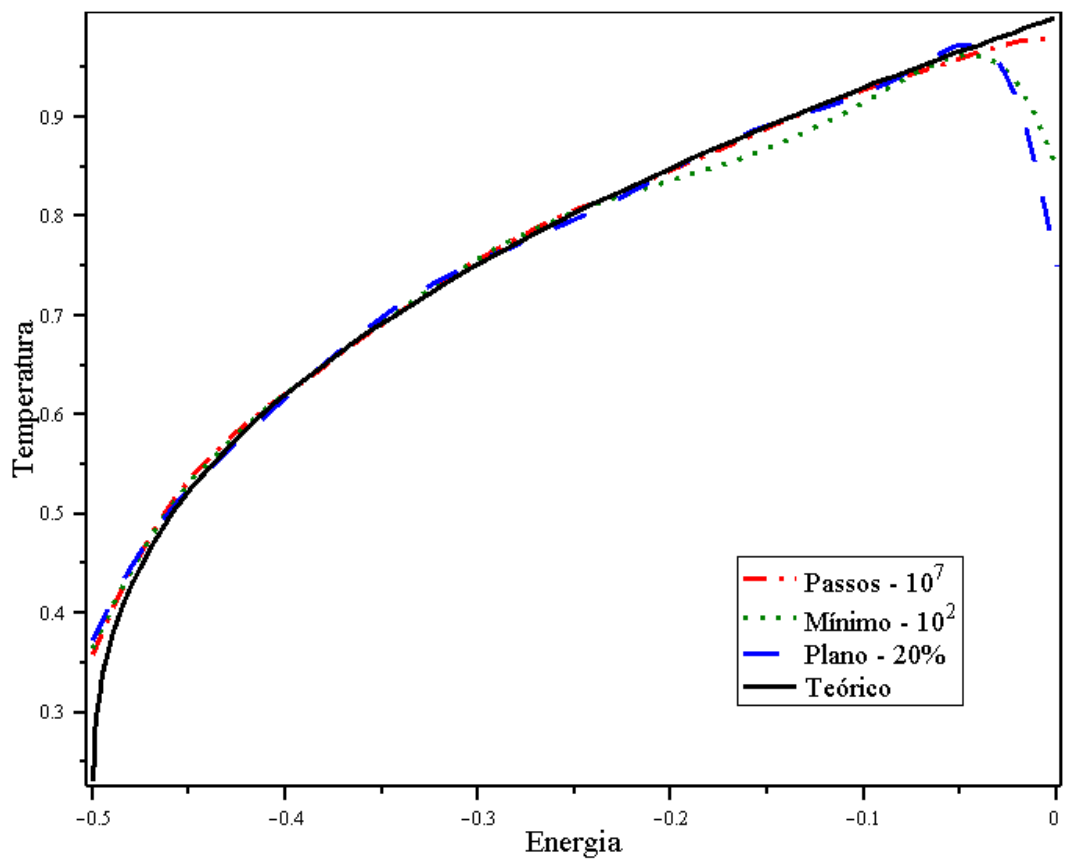

(a) Temperatura (ferromagnético)

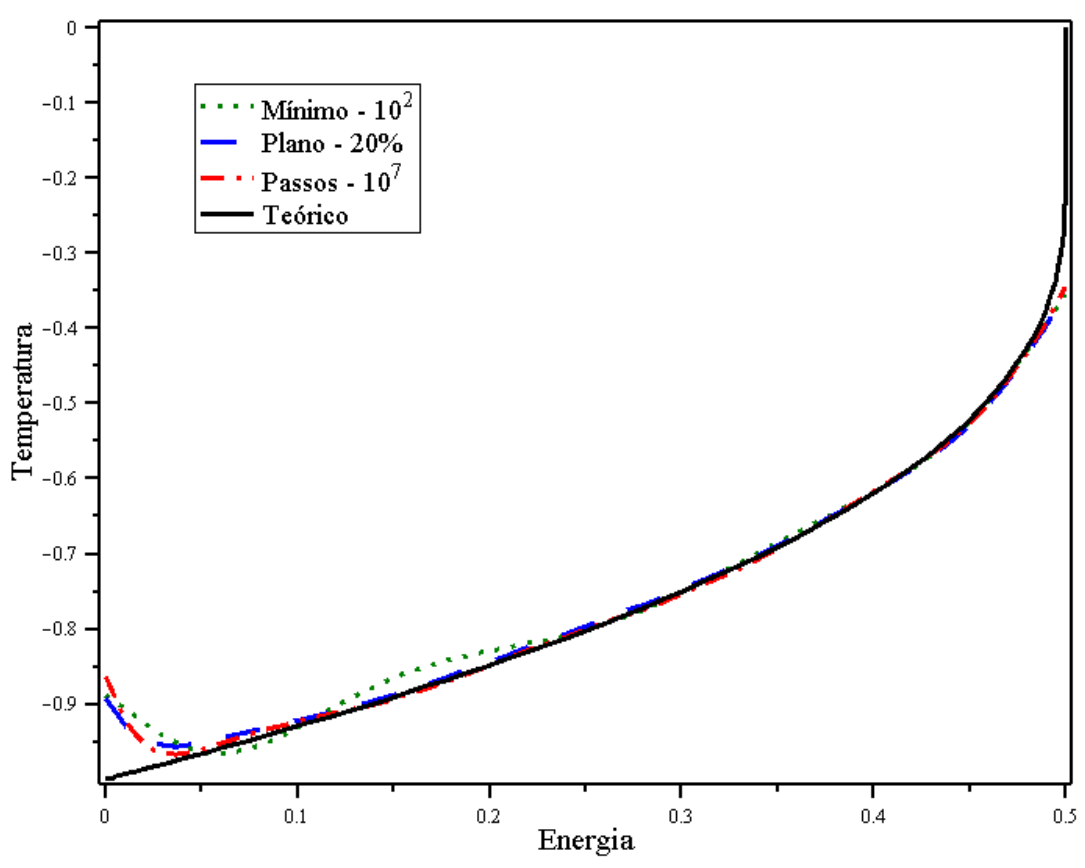

(b) Temperatura (antiferromagnético)

Figura 4.3: (a) Temperatura vs. Energia (ferromagnético). (b) Temperatura vs. Energia (antiferromagnético) para o modelo de Curie-Weiss. 


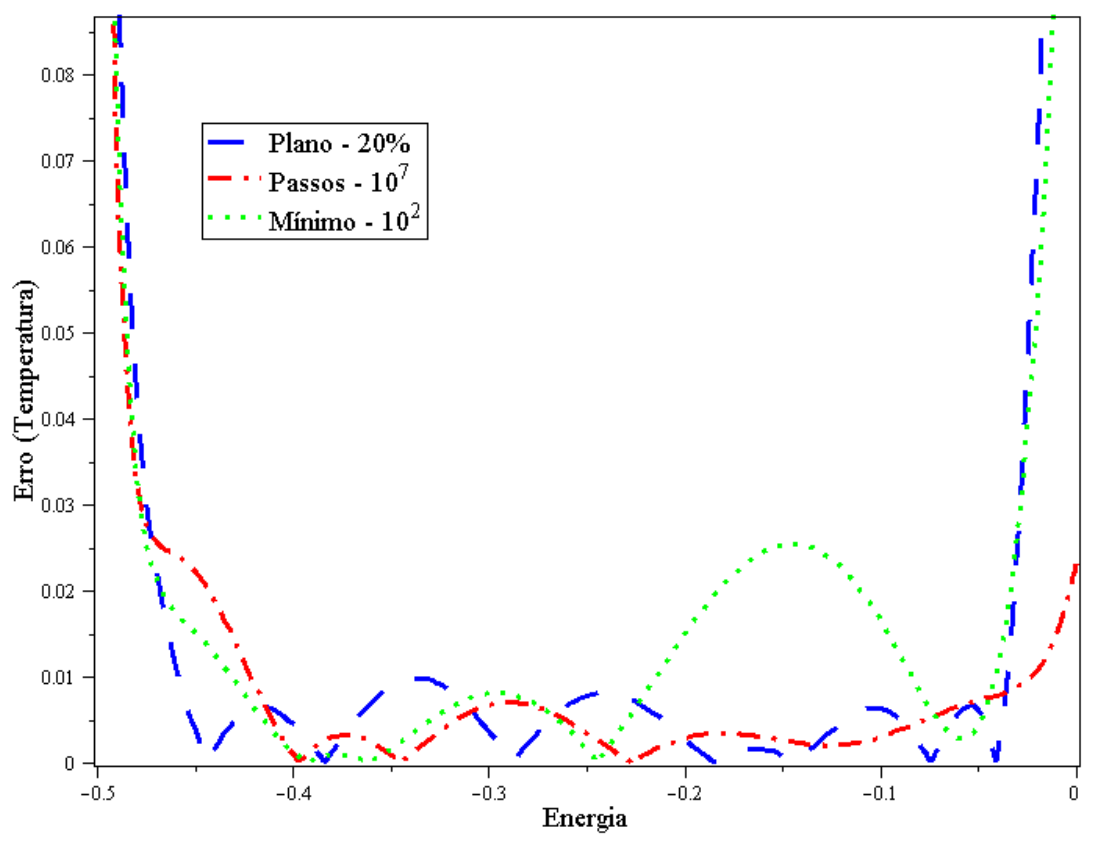

(a) Erro Temperatura (ferromagnético)

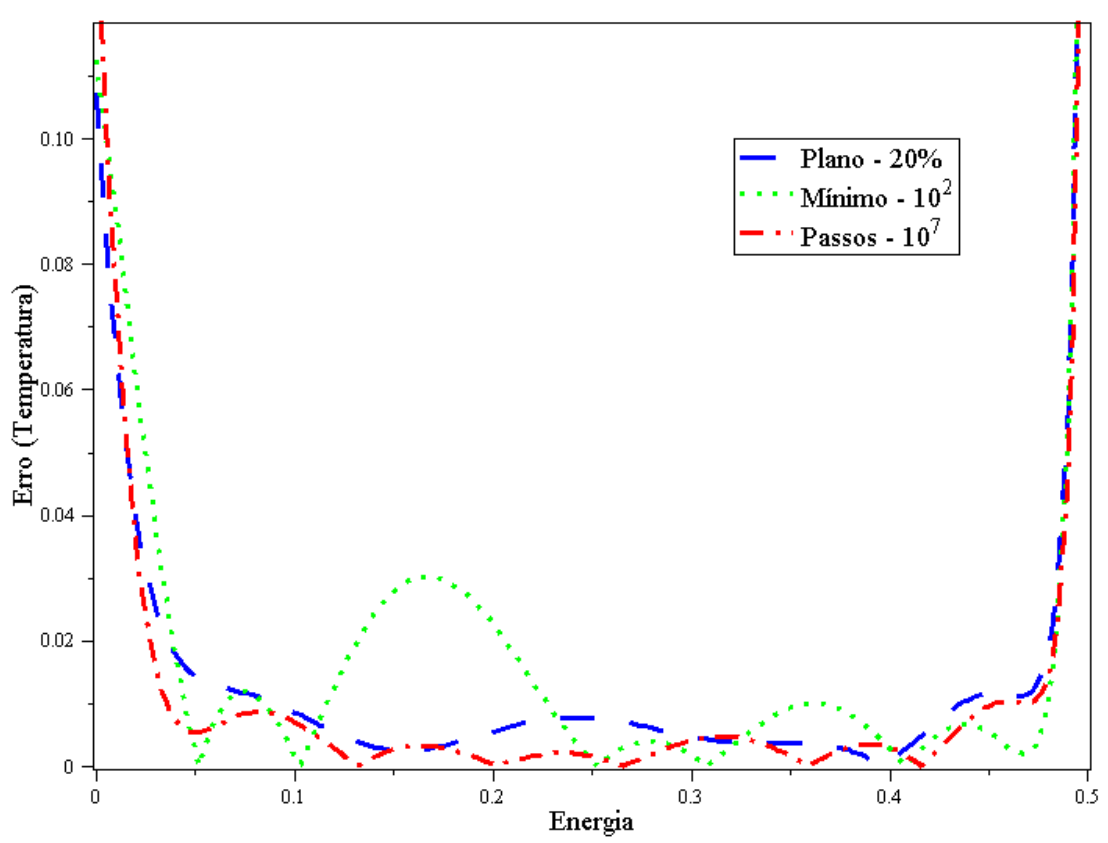

(b) Erro Temperatura (antiferromagnético)

Figura 4.4: (a) Erro da temperatura vs. Energia (ferromagnético). (b) Erro da temperatura vs. Energia (antiferromagnético) para o modelo de Curie-Weiss. 


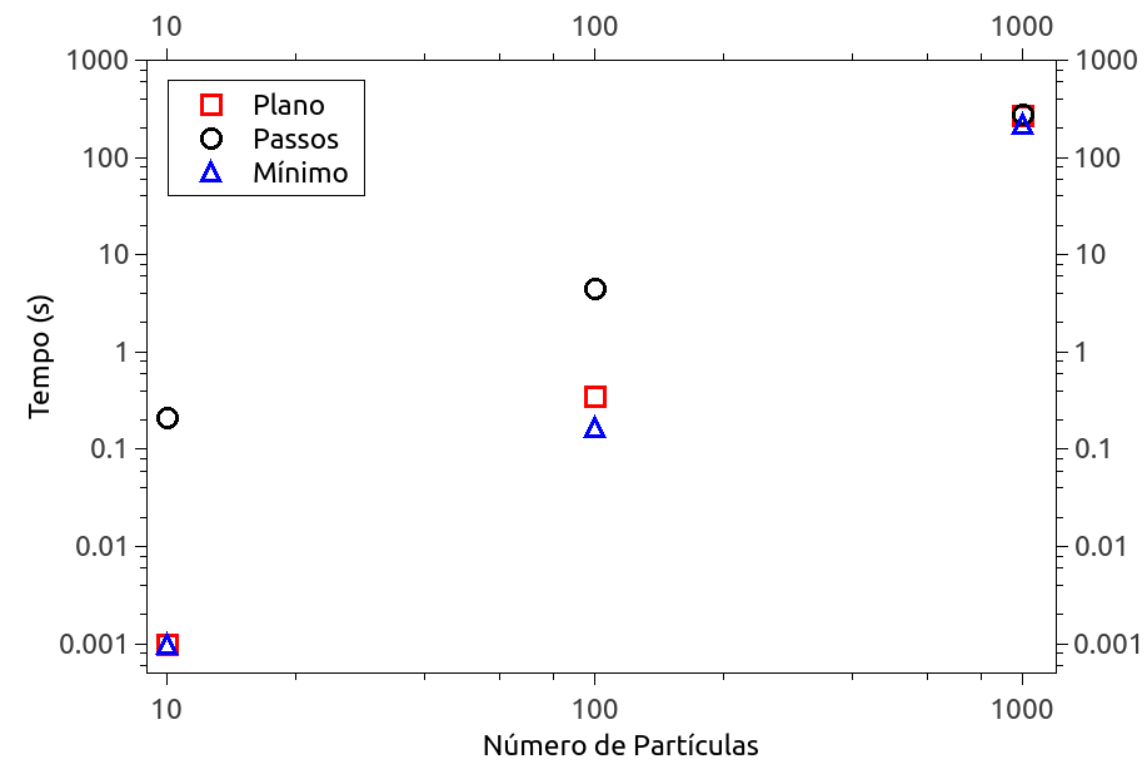

Figura 4.5: Comparação de tempos de execução para diferentes critérios do método de Wang-Landau.

\subsection{HMF 1D}

O modelo HMF-1D é apresentado na seção 2.4 como partículas movendo-se em um círculo unitário interagindo por um potencial de alcance infinito. A hamiltoniana do modelo é dada por

$$
\begin{aligned}
H_{N} & =\sum_{i=1}^{N} \frac{p_{i}^{2}}{2}+\frac{1}{2 N} \sum_{i, j=1}^{N}\left[1-\cos \left(\theta_{i}-\theta_{j}\right)\right] \\
& =\sum_{i=1}^{N} \frac{p_{i}^{2}}{2}+\frac{N}{2}\left[1-m^{2}\right],
\end{aligned}
$$

com $N$ sendo o número de partículas e $m=\sqrt{m_{x}^{2}+m_{y}^{2}}$, a magnetização intensiva, onde

$$
m_{x}=\frac{1}{N} \sum_{i=1}^{N} \cos \theta_{i} \quad e \quad m_{y}=\frac{1}{N} \sum_{i=1}^{N} \sin \theta_{i} .
$$

Como trata-se de um sistema contínuo, devemos obter a função de partição microcanônica, (1.51). Sejam $K$ e $U$ as energias cinética e potencial, respectivamente, de um sistema com energia total E. O número de microestados desse sistema é dado por, [6], 


$$
\begin{aligned}
W_{N}(E) & =\int \delta\left(E-H_{N}\right) \prod_{i=1}^{N} d p_{i} d \theta_{i}= \\
& =\int \prod_{i=1}^{N} d p_{i} d \theta_{i} \underbrace{\int d K \delta\left(K-\sum_{j=1}^{N} \frac{p_{j}^{2}}{2}\right)}_{=1} \delta\left(E-K-U\left(\left\{\theta_{i}\right\}\right)\right)= \\
& =\int d K \underbrace{\int \prod_{i=1}^{N} d p_{i} \delta\left(K-\sum_{j=1}^{N} \frac{p_{j}^{2}}{2}\right)}_{W_{\text {cin }}(K)} \underbrace{\int \prod_{i=1}^{N} d \theta_{i} \delta\left(E-K-U\left(\left\{\theta_{i}\right\}\right)\right)}_{W_{\text {conf }}(E-K)} \\
& =\int d K W_{\text {cin }}(K) W_{\text {conf }}(E-K) .
\end{aligned}
$$

Utilizando uma transformada de Laplace para resolver $W_{c i n}(K)$, temos

$$
\begin{aligned}
Z_{\text {cin }}(\beta) & =\int_{0}^{\infty} e^{-\beta K} W_{\text {cin }}(K) d K= \\
& =\int_{0}^{\infty} \int_{-\infty}^{\infty} d K \prod_{i=1}^{N} d p_{i} e^{-\beta K} \delta\left(K-\sum_{j=1}^{N} \frac{p_{j}^{2}}{2}\right)= \\
& =\int_{-\infty}^{\infty} \prod_{i=1}^{N} d p_{i} \exp \left(-\beta \sum_{j=1}^{N} \frac{p_{j}^{2}}{2}\right)= \\
& =\int_{-\infty}^{\infty} \prod_{i=1}^{N} d p_{i} \prod_{j=1}^{N} \exp \left(-\beta \frac{p_{j}^{2}}{2}\right)= \\
& =\int_{-\infty}^{\infty} d p_{1} \exp \left(-\beta \frac{p_{1}^{2}}{2}\right) \int_{-\infty}^{\infty} d p_{2} \exp \left(-\beta \frac{p_{2}^{2}}{2}\right) \cdots \int_{-\infty}^{\infty} d p_{N} \exp \left(-\beta \frac{p_{N}^{2}}{2}\right)= \\
& =\left[\int_{-\infty}^{\infty} d p \exp \left(-\beta \frac{p^{2}}{2}\right)\right]^{N} \cdot
\end{aligned}
$$

Usando $\int_{-\infty}^{\infty} e^{-\alpha x^{2}} d x=\sqrt{\frac{\pi}{\alpha}}$ em (4.16), temos que

$$
Z_{\text {cin }}(\beta)=\left(\frac{2 \pi}{\beta}\right)^{\frac{N}{2}}
$$

Aplicando a transformada inversa de Laplace $\mathcal{L}^{-1}\left\{\frac{\Gamma(n+1)}{s^{n+1}}\right\}=t^{n} \Theta(t)$, em (4.17), obtemos 


$$
\begin{aligned}
W_{\text {cin }}(K) & =\mathcal{L}^{-1}\left\{Z_{\text {cin }}(K)\right\}=\mathcal{L}^{-1}\left\{\left(\frac{2 \pi}{\beta}\right)^{\frac{N}{2}}\right\}= \\
& =\frac{(2 \pi)^{\frac{N}{2}}}{\Gamma\left(\frac{N}{2}\right)} \mathcal{L}^{-1}\left\{\frac{\Gamma\left(\frac{N}{2}\right)}{\beta^{\frac{N}{2}}}\right\}=\frac{(2 \pi)^{\frac{N}{2}}}{\Gamma\left(\frac{N}{2}\right)} K^{\frac{N}{2}-1} \Theta(K)
\end{aligned}
$$

como $K \geq 0$,

$$
\begin{gathered}
W_{\text {cin }}(K)=\frac{(2 \pi)^{\frac{N}{2}}}{\Gamma\left(\frac{N}{2}\right)} K^{\frac{N}{2}-1} \\
\therefore \ln W_{\text {cin }}(K)=\frac{N}{2} \ln 2 \pi+\left(\frac{N}{2}-1\right) \ln K-\ln \Gamma\left(\frac{N}{2}\right),
\end{gathered}
$$

usando a expressão assintótica da função $\Gamma, \ln \Gamma(N) \simeq\left(N-\frac{1}{2}\right) \ln N-N+\frac{1}{2} \ln 2 \pi$, em $(4.18)$,

$$
\ln W_{\text {cin }}(K) \simeq \frac{N}{2}\left[\ln 2 \pi+\left(1-\frac{2}{N}\right) \ln K-\left(1-\frac{1}{N}\right) \ln \frac{N}{2}+1-\frac{1}{N} \ln 2 \pi\right]
$$

Desprezando os termos que vão a zero no limite em que $N \rightarrow \infty$, a equação (4.19) se torna

$$
\begin{gathered}
\ln W_{\text {cin }}(K) \simeq \frac{N}{2}\left[1+\ln 4 \pi+\ln \frac{K}{N}\right] \\
\therefore W_{\text {cin }}(K) \simeq \exp \left[\frac{N}{2}\left(1+\ln 4 \pi+\ln \frac{K}{N}\right)\right] .
\end{gathered}
$$

Substituindo (4.20) em (4.15)

$$
W_{N}(E)=\int d K \exp \left[\frac{N}{2}\left(1+\ln 4 \pi+\ln \frac{K}{N}\right)\right] W_{\text {conf }}(E-K) .
$$

Definindo a densidade de energia total, cinética e potencial como $e=\frac{E}{N}, k=\frac{K}{N}$ e $u=\frac{U}{N}$, respectivamente, e definindo também a entropia configuracional como $s_{\text {conf }}(e-k)=$ $\frac{1}{N} \ln W_{\text {conf }}(E-K)$, onde $e-k=u$. A função de partição microcanônica (4.21) pode ser reescrita como 


$$
W_{N}(N e)=N \int d k \exp \left[N\left(\frac{1}{2}+\frac{1}{2} \ln 4 \pi+\frac{1}{2} \ln k+s_{\text {conf }}(e-k)\right)\right]
$$

A expressão (4.22) nos dá o número de microestados do sistema com uma energia total $E=N e$. Podemos ver a integração em $k$ como a soma de todos os microestados com uma energia total $E=N e$ e energia cinética $K=N k$, ou seja,

$$
W_{N}(N e)=\int d(N k) W_{N}(N e, N k)
$$

Comparando (4.23) com (4.22), temos que

$$
W_{N}(N e, N k)=\exp \left[N\left(\frac{1}{2}+\frac{1}{2} \ln 4 \pi+\frac{1}{2} \ln k+s_{\text {conf }}(e-k)\right)\right] .
$$

Substituindo $k=e-u$, em (4.24), temos o número de microestados do sistema com energia total $E=N e$ e energia potencial $U=N u$,

$$
W_{N}(N e, N u)=\exp \left[N\left(\frac{1}{2}+\frac{1}{2} \ln 4 \pi+\frac{1}{2} \ln (e-u)+s_{\text {conf }}(u)\right)\right] .
$$

Com isso, a entropia por partícula, em função de e e u, é obtida aplicando o limite termodinâmico

$$
s(e, u)=\lim _{N \rightarrow \infty} \frac{1}{N} \ln W_{N}(N e, N u)=\frac{1}{2}+\frac{1}{2} \ln 4 \pi+\frac{1}{2} \ln (e-u)+s_{\text {conf }}(u) .
$$

Uma forma de se obter $s(e)$, é maximizando a expressão (4.26) em relação a $u$, para isso é necessário ter uma expressão explícita da entropia configuracional $s_{c o n f}(u)$, ou seja, é preciso calcular $W_{\text {conf }}(U)$.

No caso do HMF-1D, da equação (4.13), $U$ e $|m|$ possuem uma correspondência um para um, ou seja, definindo

$$
W(m)=\int \prod_{i} d \theta_{i} \delta\left(\sum_{i} \cos \theta_{i}-N m\right) \delta\left(\sum_{i} \sin \theta_{i}\right),
$$

temos que $W(m)$ é proporcional a $W_{\text {conf }}(U)$ (a constante de proporcionalidade desaparece no limite termodinâmico), [6]. Existe uma degenerescência contínua da fase do sistema, portanto, não há perda de generalidade em definir a magnetização na direção do eixo $x$, 
como foi feito na expressão (4.27). Usando a representação de Fourier da função $\delta$ em (4.27), temos

$$
\begin{aligned}
& W(m)=\left(\frac{1}{2 \pi}\right)^{2} \int_{-\infty}^{\infty} d q_{2} \int_{-\infty}^{\infty} d q_{1} \int \prod_{i} d \theta_{i} \exp \left[i q_{1}\left(\sum_{i} \cos \theta_{i}-N m\right)\right] \times \\
& \times \exp \left[i q_{1}\left(\sum_{i} \sin \theta_{i}\right)\right]= \\
& =\left(\frac{1}{2 \pi}\right)^{2} \int_{-\infty}^{\infty} d q_{2} \int_{-\infty}^{\infty} d q_{1} \int \prod_{i} d \theta_{i} e^{-i q_{1} N m} \exp \left(i q_{1} \sum_{i} \cos \theta_{i}+i q_{2} \sum_{i} \sin \theta_{i}\right)= \\
& =\left(\frac{1}{2 \pi}\right)^{2} \int_{-\infty}^{\infty} d q_{2} \int_{-\infty}^{\infty} d q_{1} \int \prod_{i} d \theta_{i} e^{-i q_{1} N m} \exp \left[\sum_{i}\left(i q_{1} \cos \theta_{i}+i q_{2} \sin \theta_{i}\right)\right]= \\
& =\left(\frac{1}{2 \pi}\right)^{2} \int_{-\infty}^{\infty} d q_{2} \int_{-\infty}^{\infty} d q_{1} e^{-i q_{1} N m} \int \prod_{i} d \theta_{i}\left[e^{i q_{1} \cos \theta_{1}+i q_{2} \sin \theta_{1}}\right] \cdots\left[e^{i q_{1} \cos \theta_{N}+i q_{2} \sin \theta_{N}}\right] \\
& =\left(\frac{1}{2 \pi}\right)^{2} \int_{-\infty}^{\infty} d q_{1} e^{-i q_{1} N m} \int_{-\infty}^{\infty} d q_{2}\left[\int_{0}^{2 \pi} d \theta e^{i\left(q_{1} \cos \theta+q_{2} \sin \theta\right)}\right]^{N} .
\end{aligned}
$$

Usando $a \cos x+b \sin x=R \cos (x-\alpha)$, onde $R=\sqrt{a^{2}+b^{2}}$ e $\alpha=\tan ^{-1}\left(\frac{b}{a}\right)$, ficamos com

$$
W(m)=\left(\frac{1}{2 \pi}\right)^{2} \int_{-\infty}^{\infty} d q_{1} \int_{-\infty}^{\infty} d q_{2} e^{-i q_{1} N m} J_{0}^{N}\left(\left(q_{1}^{2}+q_{2}^{2}\right)^{\frac{1}{2}}\right),
$$

onde $J_{0}(z)$ é a função de Bessel de ordem zero,

$$
J_{0}(z)=\int_{0}^{2 \pi} d \theta e^{i z \cos \theta}
$$

Com isso, podemos escrever (4.29) como

$$
W(m)=\left(\frac{1}{2 \pi}\right)^{2} \int_{-\infty}^{\infty} \int_{-\infty}^{\infty} d q_{1} d q_{2} e^{N f\left(q_{1}, q_{2}\right)},
$$

$\operatorname{com} f\left(q_{1}, q_{2}\right)=-i q_{1} m+\ln J_{0}\left(\left(q_{1}^{2}+q_{2}^{2}\right)^{\frac{1}{2}}\right)$. Podemos aproximar a integral em (4.31) pelo valor máximo do integrando, devido ao comportamento da função exponencial. Usando o fato de que a derivada de $J_{0}$ é $-J_{1}$ (função de Bessel de ordem um), para achar $q_{1}^{*}$ e $q_{2}^{*}$, que maximizam $f\left(q_{1}, q_{2}\right)$, temos que resolver o seguinte sistema:

$$
i m+\frac{J_{1}\left(\left(q_{1}^{* 2}+q_{2}^{* 2}\right)^{\frac{1}{2}}\right)}{J_{0}\left(\left(q_{1}^{* 2}+q_{2}^{* 2}\right)^{\frac{1}{2}}\right)} \frac{q_{1}^{*}}{\sqrt{q_{1}^{* 2}+q_{2}^{* 2}}}=0
$$




$$
\frac{J_{1}\left(\left(q_{1}^{* 2}+q_{2}^{* 2}\right)^{\frac{1}{2}}\right)}{J_{0}\left(\left(q_{1}^{* 2}+q_{2}^{* 2}\right)^{\frac{1}{2}}\right)} \frac{q_{2}^{*}}{\sqrt{q_{1}^{* 2}+q_{2}^{* 2}}}=0
$$

Da equação (4.33), temos que $q_{2}^{*}=0$, com isso a equação (4.32) fica

$$
\frac{J_{1}\left(q_{1}^{*}\right)}{J_{0}\left(q_{1}^{*}\right)}=-i m
$$

Definindo $q_{1}^{*}=-i \gamma$ e usando a propriedade das funções de Bessel $I_{n}(z)=i^{-n} J_{n}(i z)$, onde $I_{n}(z)$ é a função modificada de Bessel de ordem $n$, obtemos

$$
\frac{I_{1}(\gamma)}{I_{0}(\gamma)}=m
$$

Denotando por $B_{i n v}$ a função inversa de $\frac{I_{1}}{I_{0}}$, temos que $q_{1}^{*}=-i B_{i n v}(m)$. Com isso,

$$
\begin{gathered}
W(m) \simeq\left(\frac{1}{2 \pi}\right)^{2} e^{N\left[-m B_{i n v}(m)+\ln I_{0}\left(B_{i n v}(m)\right)\right]}, \\
s_{\text {conf }}(m)=\lim _{N \rightarrow \infty} \frac{1}{N} \ln W(m)=-m B_{i n v}(m)+\ln I_{0}\left(B_{i n v}(m)\right) .
\end{gathered}
$$

Substituindo (4.37) em (4.26) e usando $u=\frac{1}{2}-\frac{1}{2} m^{2}$, temos que a entropia por partícula em função de $e$ e $m$ é dada por

$$
s(e, m)=\frac{1}{2}(1+\ln 4 \pi)+\frac{1}{2} \ln \left(e-\frac{1}{2}+\frac{1}{2} m^{2}\right)-m B_{i n v}(m)+\ln I_{0}\left(B_{i n v}(m)\right) .
$$

De (4.38), temos

$$
\begin{gathered}
\frac{\partial s(e, m)}{\partial m}=\frac{m}{2 e-1+m^{2}}-B_{i n v}(m) \\
\frac{\partial s(e, m)}{\partial e}=\frac{1}{2 e-1+m^{2}}
\end{gathered}
$$

A entropia por partícula como função apenas da densidade de energia do sistema $s(e)$, é obtida através da maximização de (4.38) em relação a $m$, obtendo-se assim uma magnetização de equilíbrio $m(e)$. A magnetização espontânea $m(e)$ é obtida igualando a equação (4.39) a zero, ou seja, ela deve satisfazer 


$$
\frac{m(e)}{2 e-1+m^{2}(e)}-B_{i n v}(m(e))=0 .
$$

Uma vez obtida a entropia $s(e)$, a temperatura do sistema é dada por (utilizando as equações (4.39), (4.40) e (4.41))

$$
\begin{gathered}
\frac{d s(e)}{d e}=\frac{1}{T(e)}=\left[\frac{\partial s(e, m)}{\partial e}+\frac{\partial s(e, m)}{\partial m} \frac{\partial m}{\partial e}\right]_{m=m(e)}= \\
=\left[\frac{\partial s(e, m)}{\partial e}+\left(\frac{m}{2 e-1+m^{2}}-B_{i n v}(m)\right) \frac{\partial m}{\partial e}\right]_{m=m(e)}= \\
=\left[\frac{\partial s(e, m)}{\partial e}\right]_{m=m(e)} \\
\therefore T(e)=2 e-1+m^{2}(e) .
\end{gathered}
$$

A solução da equação (4.41) é obtida graficamente e está ilustrada na figura 4.6, para $0<e \leq \frac{3}{4}$ a magnetização $m(e)$ decresce de 1 até 0 , enquanto para $e>\frac{3}{4}$ a única solução é $m=0$. Na figura 4.6 também está a magnetização de equilíbrio encontrada com o método de Ray e de Wang-Landau.

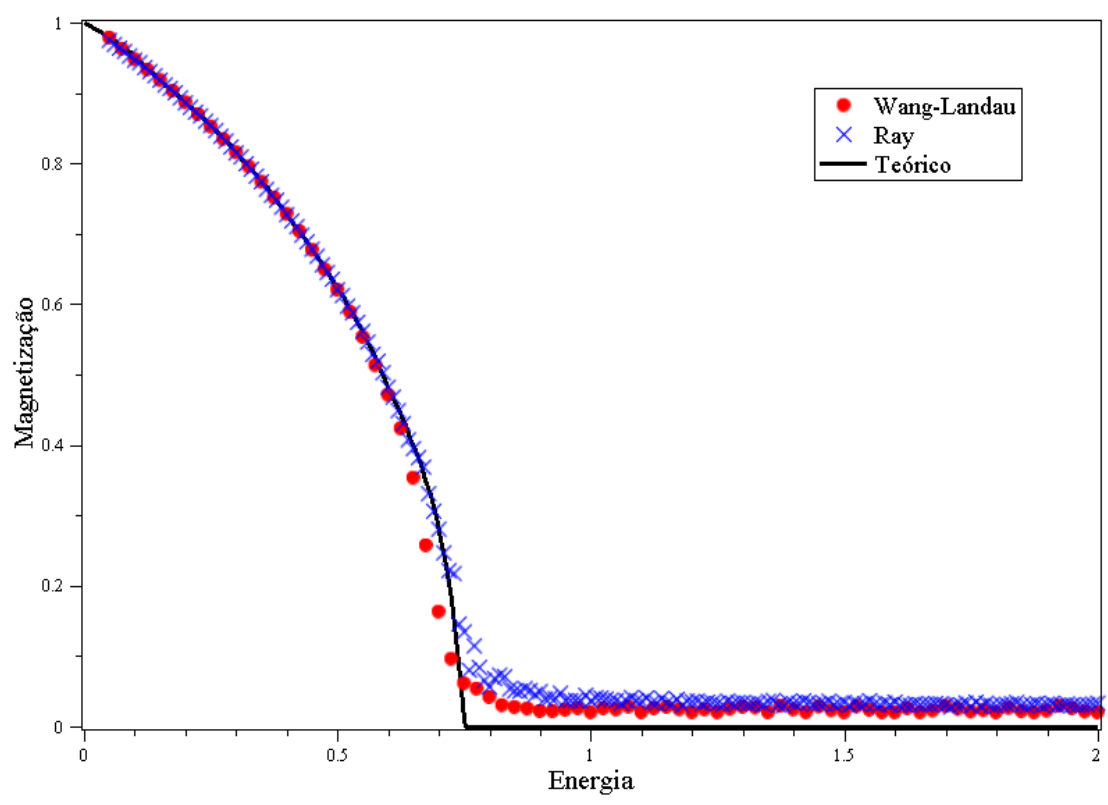

Figura 4.6: Magnetização espontânea teórica (linha sólida) e valores obtidos numericamente, utilizando dos métodos de Ray (pontos azuis) e Wang-Landau (pontos vermelhos), para o modelo HMF-1D. 
Os gráficos da entropia $s(e)$ e da temperatura $T(e)$ teóricos estão na figura 4.7 junto dos resultados numéricos utilizando os métodos de Ray e de Wang-Landau, ambos calculados com $10^{3}$ partículas. É possível notar, da figura 4.7(b), que existe uma descontinuidade na derivada da temperatura em relação a energia em $e=\frac{3}{4}$ (ou uma descontinuidade na segunda derivada da entropia em relação a energia) o que caracteriza uma transição de fase de segunda ordem. O sistema possui uma fase de equilíbrio com magnetização não nula para $0<e \leq \frac{3}{4}$, e uma fase com magnetização nula para $e>\frac{3}{4}$.

Ambos os métodos apresentam bons resultados, em comparação com o resultado analítico. As figuras 4.6 e 4.7 mostram uma quantidade de pontos muito maior para o método de Ray, em comparação ao de Wang-Landau. A razão disso vem do fato de que, diferente do método discreto, o método de Wang-Landau contínuo não calcula diretamente a entropia do sistema $s(e)$, o que ele obtem é a entropia configuracional $s(U)=\ln g(U)$, onde $U$ é a energia potencial e $g(U)$ é a densidade de estados configuracional. Substituindo $s(U)$ na equação (3.40), temos a entropia do sistema. Ou seja, após o cálculo da entropia configuracional uma integração ainda deve ser feita, para obtermos a entropia $s(e)$. Na figura 4.8 temos a entropia configuracional do modelo HMF-1D, para diversos números de partículas. Com $s(U)$ obtido pelo método de Wang-Landau é possível obter a entropia do modelo HMF-1D em função da energia e da magnetização. Da equação (4.13), temos que

$$
U(m)=\frac{N}{2}\left(1-m^{2}\right)
$$

Com isso, usando a equação (3.40)

$$
\begin{aligned}
s(e, m) & =\frac{1}{N} \ln \left\{[E-U(m)]^{\frac{N}{2}-1} \exp [s(U(m))]\right\} \\
& =\frac{1}{N} \ln \left\{\left[E-\frac{N}{2}\left(1-m^{2}\right)\right]^{\frac{N}{2}-1} \exp \left[s\left(\frac{N}{2}\left(1-m^{2}\right)\right)\right]\right\}
\end{aligned}
$$

O gráfico da entropia como função da energia e da magnetização está na figura 4.9. É possível notar que para baixas energias, $e<0.75$, os valores da magnetização que maximizam a entropia são não nulos, enquanto que para energias altas, $e \geq 0.75$, os valores da magnetização que maximizam a entropia são todos zero. As comparações de desempenho dos métodos é feita na seção 4.4 . 


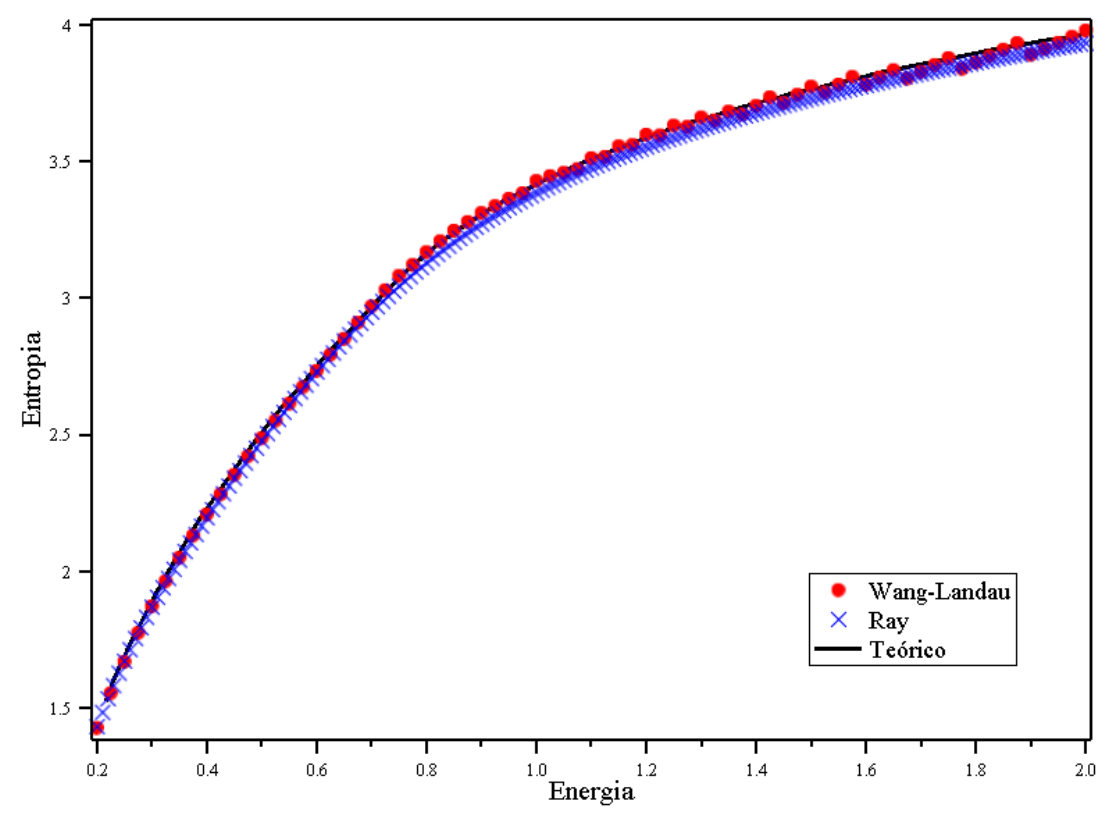

(a) Entropia HMF-1D

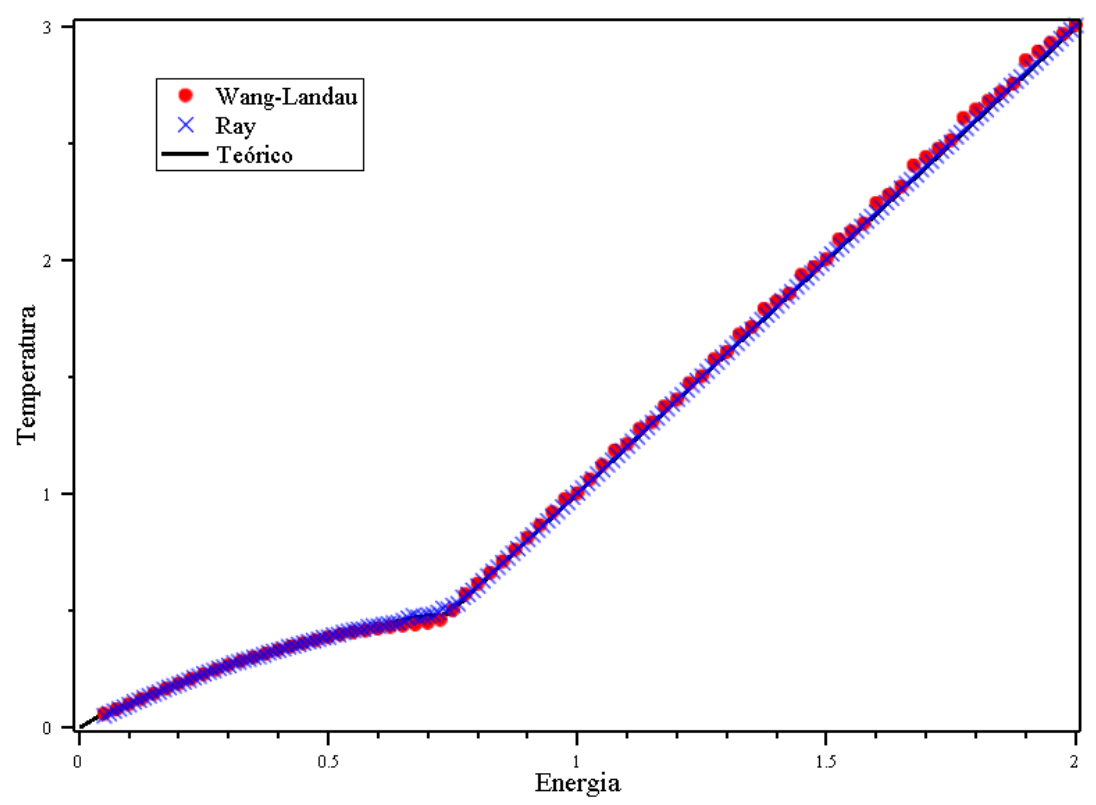

(b) Temperatura HMF-1D

Figura 4.7: (a) Entropia teórica (linha sólida) e valores obtidos numericamente, utilizando os métodos de Ray (pontos azuis) e Wang-Landau (pontos vermelhos), para o modelo HMF-1D. (b) Temperatura teórica (linha sólida) e valores obtidos numericamente, utilizando os métodos de Ray (pontos azuis) e Wang-Landau (pontos vermelhos), para o modelo HMF-1D. 


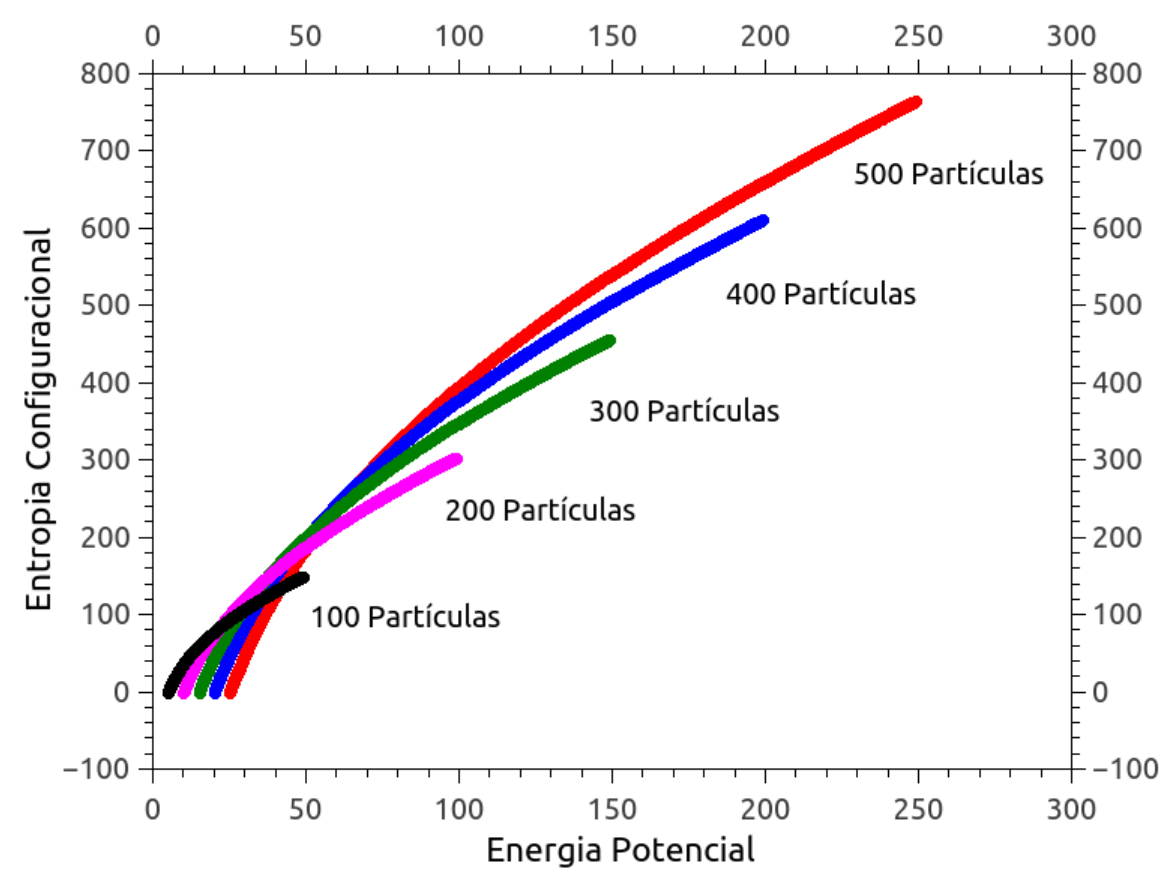

Figura 4.8: Entropia configuracional do modelo HMF-1D.

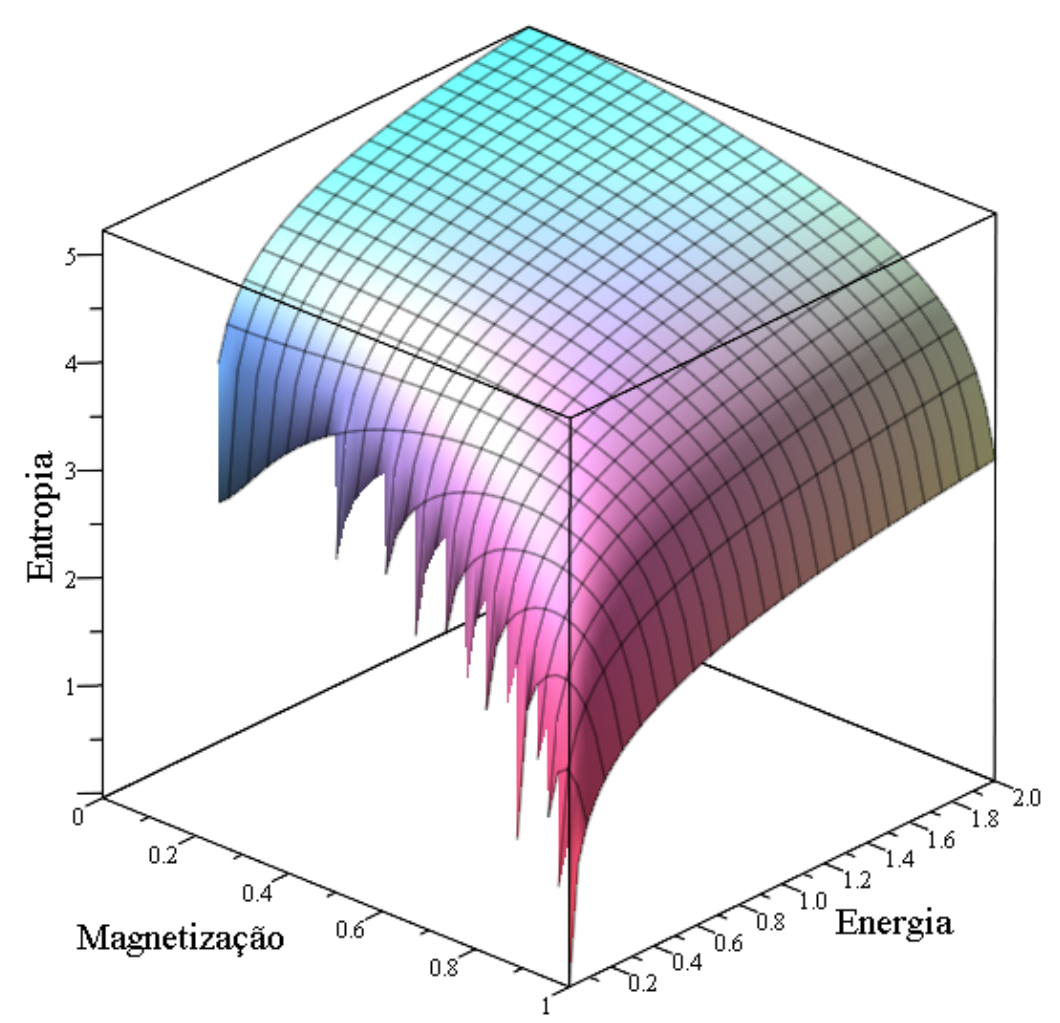

Figura 4.9: Entropia do modelo HMF-1D em função da energia e magnetização. 


\subsection{HMF 2D}

O modelo HMF-2D é apresentado na seção 2.4, com a hamiltoniana dada por

$$
\begin{aligned}
H_{N}= & \sum_{i=1}^{N} \frac{p_{i \theta}^{2}+p_{i \phi}^{2}}{2}+\frac{1}{2 N} \sum_{i, j=1}^{N}\left[3-\cos \left(\theta_{i}-\theta_{j}\right)-\right. \\
& \left.-\cos \left(\phi_{i}-\phi_{j}\right)-\cos \left(\theta_{i}-\theta_{j}\right) \cos \left(\phi_{i}-\phi_{j}\right)\right]= \\
= & \sum_{i=1}^{N} \frac{p_{i \theta}^{2}+p_{i \phi}^{2}}{2}+\frac{N}{2}\left[3-m_{\theta}^{2}-m_{\phi}^{2}-\frac{1}{2}\left(P_{+}^{2}+P_{-}^{2}\right)\right]
\end{aligned}
$$

onde, para cada partícula $i, p_{i \theta}$ é o momento conjugado a $\theta_{i}, p_{i \phi}$ é o momento conjugado a $\phi_{i}$. As magnetizações $m_{\theta}$ e $m_{\phi}$ e as polarizações $P_{+}$e $P_{-}$são dados por

$$
\begin{gathered}
m_{\theta}^{2}=\left(\sum_{i=1}^{N} \frac{\cos \theta_{i}}{N}\right)^{2}+\left(\sum_{i=1}^{N} \frac{\sin \theta_{i}}{N}\right)^{2}, \\
m_{\phi}^{2}=\left(\sum_{i=1}^{N} \frac{\cos \phi_{i}}{N}\right)^{2}+\left(\sum_{i=1}^{N} \frac{\sin \phi_{i}}{N}\right)^{2}, \\
P_{+}^{2}=\left(\sum_{i=1}^{N} \frac{\cos \left(\theta_{i}+\phi_{i}\right)}{N}\right)^{2}+\left(\sum_{i=1}^{N} \frac{\sin \left(\theta_{i}+\phi_{i}\right)}{N}\right)^{2}, \\
P_{-}^{2}=\left(\sum_{i=1}^{N} \frac{\cos \left(\theta_{i}-\phi_{i}\right)}{N}\right)^{2}+\left(\sum_{i=1}^{N} \frac{\sin \left(\theta_{i}-\phi_{i}\right)}{N}\right)^{2},
\end{gathered}
$$

Neste trabalho não é apresentada uma solução analitíca de equilíbrio para o HMF-2D, mas existe uma estimativa analítica da curva calórica feita por M. Antoni e A. Torcini, [3], que indica uma transição de fase de segunda ordem com calor específico negativo em $e \approx 2$ e $T \approx 0.5$. Os gráficos da entropia e da temperatura do modelo HMF-2D, obtidos pelo método de Ray e de Wang-Landau, estão na figura 4.10 e estão de acordo com resultados numéricos prévios, $[2,14,19,24]$. Assim como no caso do HMF-1D, uma integração deve ser feita depois que a entropia configuracional $s(U)$ é obtida, pelo método de Wang-Landau. Na figura 4.11 está a entropia configuracional do modelo HMF-2D, para diversos números de partículas. As magnetizações e polarizações, calculadas pelo método de Ray usando as equações (4.46)-(4.49), estão na figura 4.12. As comparações de desempenho dos métodos é feita na seção 4.4 . 


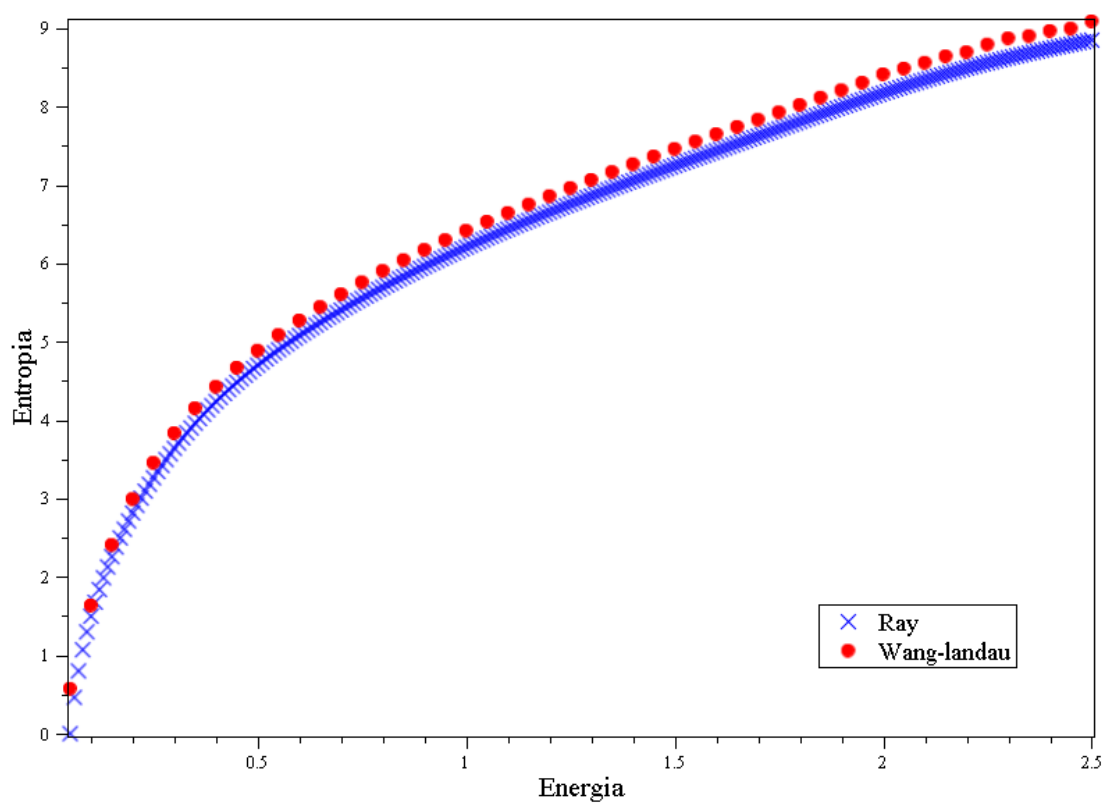

(a) Entropia HMF-2D

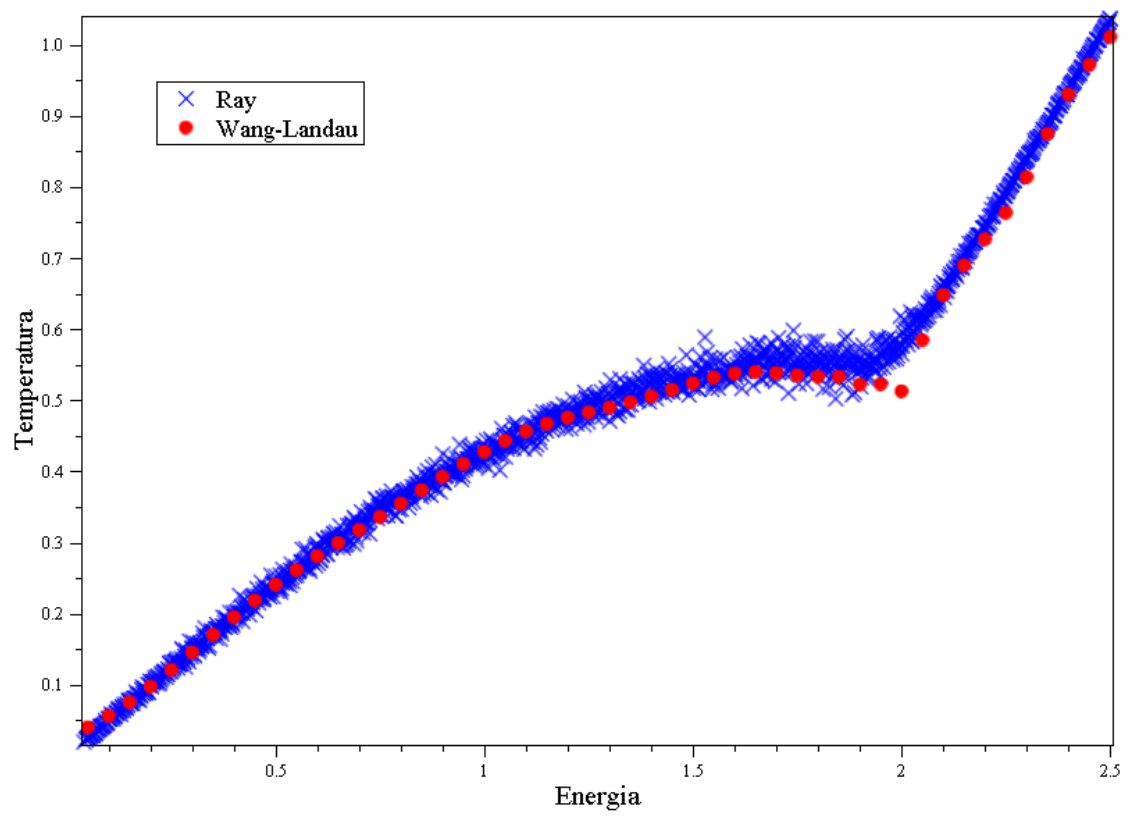

(b) Temperatura HMF-2D

Figura 4.10: (a) Entropia obtida numericamente, utilizando os métodos de Ray (pontos azuis) e Wang-Landau (pontos vermelhos), para o modelo HMF-2D. (b) Temperatura obtida numericamente, utilizando os métodos de Ray (pontos azuis) e Wang-Landau (pontos vermelhos), para o modelo HMF-2D. 


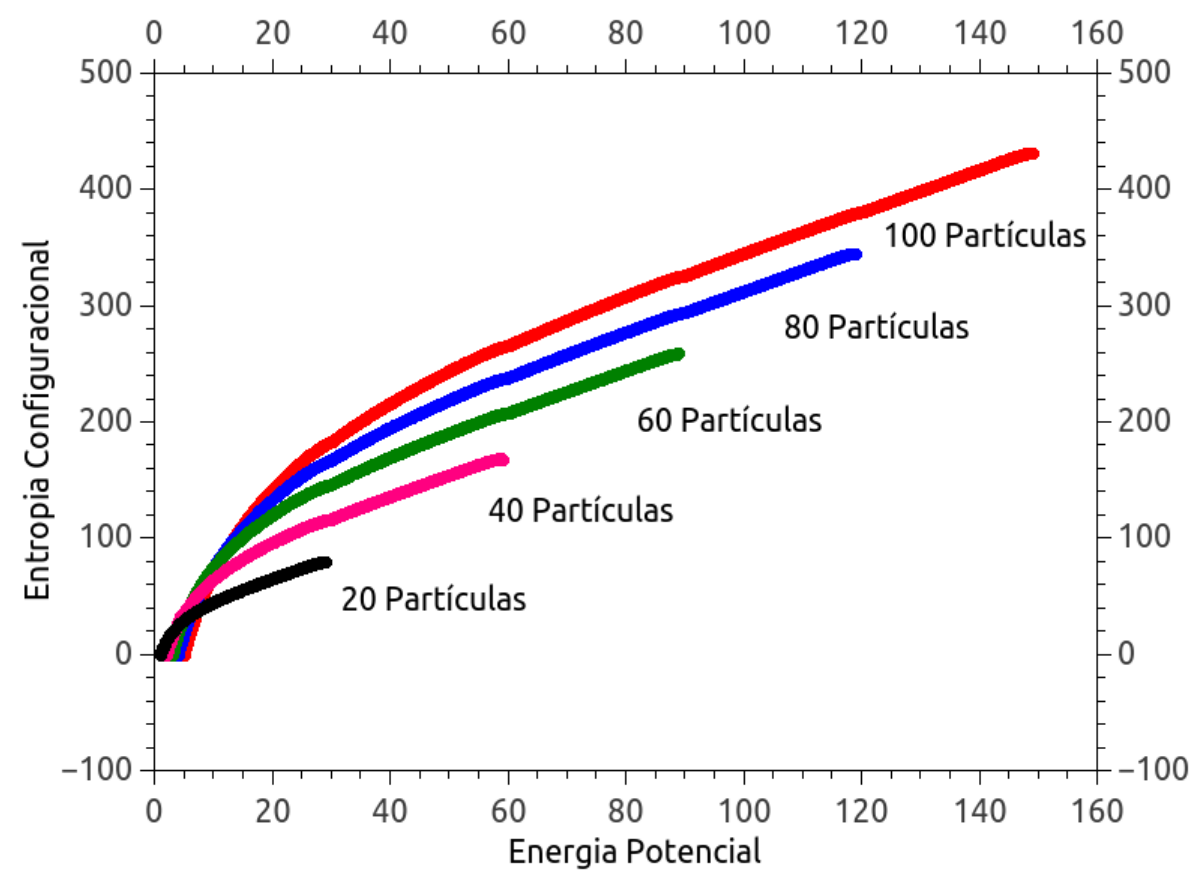

Figura 4.11: Entropia configuracional do modelo HMF-2D.

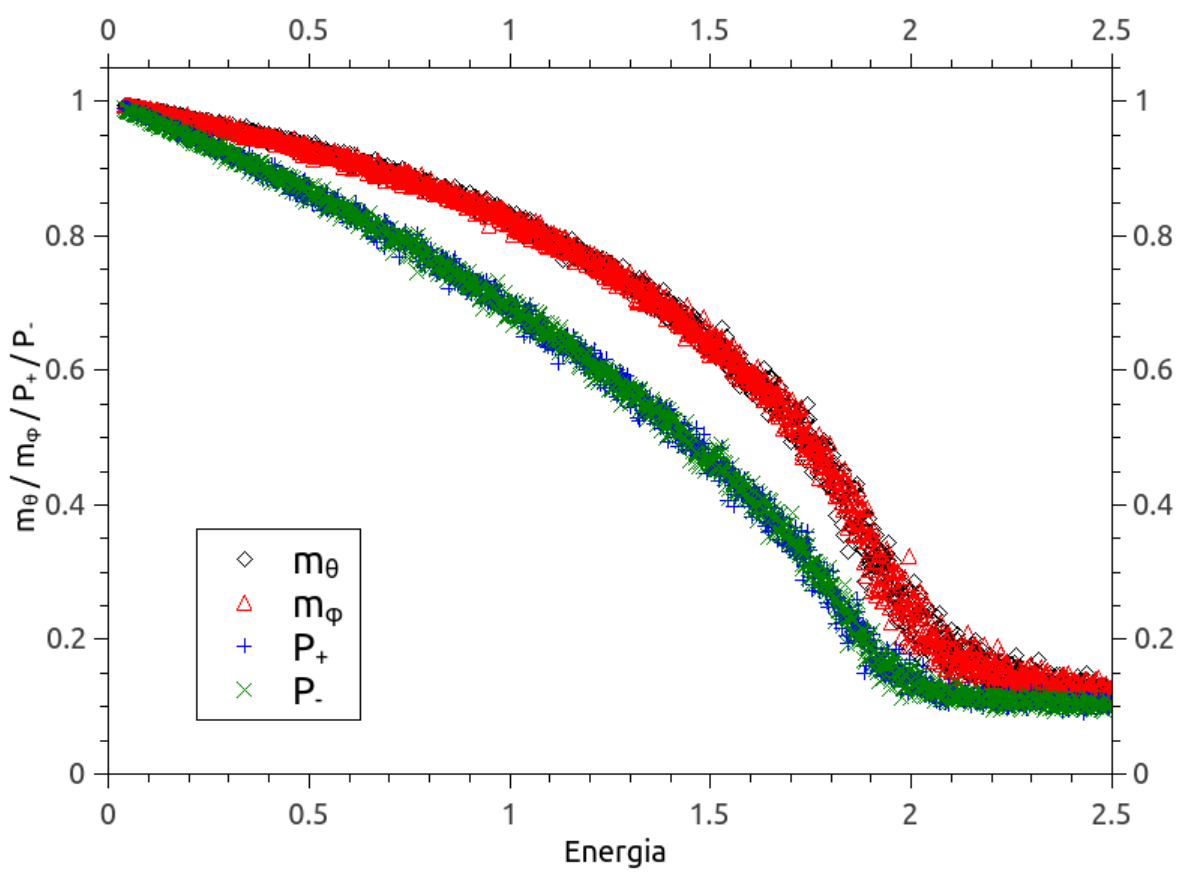

Figura 4.12: Magnetizações e polarizações do modelo HMF-2D. 


\subsection{Discussões}

Existem diferenças notáveis entre os métodos de Ray e Wang-Landau. A primeira delas sendo o fato de que o método de Wang-Landau pode ser aplicado em sistemas discretos, o que não é possível com o método de Ray. Isso vem do fato de que a probabilidade de aceitação do método de Wang-Landau, assim como no método de Metropolis, depende apenas da energia potencial $U$, que pode ser substituída pela energia total $E$ para sistemas discretos. Para o método de Ray, a probabilidade de aceitação depende da energia total e da energia potencial, tornando a simples substituição de $U$ por $E$ inapropriada. No caso de sistemas contínuos, os dois métodos possuem diferenças a respeito da aplicabilidade.

No cálculo numérico do HMF-1D, ambos os métodos mostram boa concordância com os resultados teóricos para entropia, temperatura e magnetização (Figuras 4.6 e 4.7). Com o método de Ray obtemos a distribuição de posições $\left\{\theta_{i}\right\}$ e com isso é possível calcular a magnetização, pela equação (4.14), e a temperatura, pela equação (3.24). A entropia é obtida através da temperatura por:

$$
s(e)=\int \frac{1}{T(e)} d e
$$

Através do método de Wang-Landau obtemos a densidade de estados configuracional $g(U)$ e usando a equação (3.40) obtemos a entropia do sistema. Uma vez obtida a entropia $s(e)$, tem-se a temperatura através de

$$
T(e)=\left(\frac{d s(e)}{d e}\right)^{-1}
$$

A magnetização é obtida da equação (4.42) utilizando $T(e)$. Pelo método de WangLandau, ainda é possível obter a entropia como função da energia e magnetização pela equação (3.40), uma vez que a energia potencial possui uma relação de um para um com o módulo da magnetização, equação (4.43). Resumindo, todas as informações que podem ser extraídas do método de Ray são derivadas da distribuição de posições, enquanto que as informações extraídas do método de Wang-Landau vem da densidade de estados.

O tempo de execução de cada programa depende de diversos fatores como número de partículas, número de passos e número de pontos calculados. Fixando os mesmos números de pontos calculados e números de passos para ambos os métodos, o tempo de execução dos programas para cada algoritmo, no modelo HMF-1D, encontram-se na tabela 4.2. O tempo de execução em função do número de partículas, para número de passos e pontos 


\begin{tabular}{|c|c|c|c|c|c|c|}
\hline \multirow{2}{*}{$\begin{array}{c}\text { Número de } \\
\text { Partículas }\end{array}$} & \multicolumn{3}{|c|}{ Ray } & \multicolumn{3}{|c|}{ Wang-Landau } \\
\cline { 2 - 7 } & Tempo (s) & $\begin{array}{c}\text { Número } \\
\text { de Passos }\end{array}$ & $\begin{array}{c}\text { Número } \\
\text { de Pontos }\end{array}$ & Tempo (s) & $\begin{array}{c}\text { Número } \\
\text { de Passos }\end{array}$ & $\begin{array}{c}\text { Número } \\
\text { de Pontos }\end{array}$ \\
\hline 100 & 123.42 & $10^{5}$ & $10^{2}$ & 5.65 & $10^{5}$ & $10^{2}$ \\
\hline 200 & 228.24 & $10^{5}$ & $10^{2}$ & 8.75 & $10^{5}$ & $10^{2}$ \\
\hline 300 & 337.54 & $10^{5}$ & $10^{2}$ & 12.71 & $10^{5}$ & $10^{2}$ \\
\hline 400 & 477.86 & $10^{5}$ & $10^{2}$ & 17.57 & $10^{5}$ & $10^{2}$ \\
\hline 500 & 624.31 & $10^{5}$ & $10^{2}$ & 22.22 & $10^{5}$ & $10^{2}$ \\
\hline
\end{tabular}

Tabela 4.2: Tempos de execução com número de passos e pontos fixados para diferentes números de partículas dos métodos de Ray e Wang-Landau aplicados ao modelo HMF-1D.

fixados, da tabela 4.2 estão na figura 4.13 .

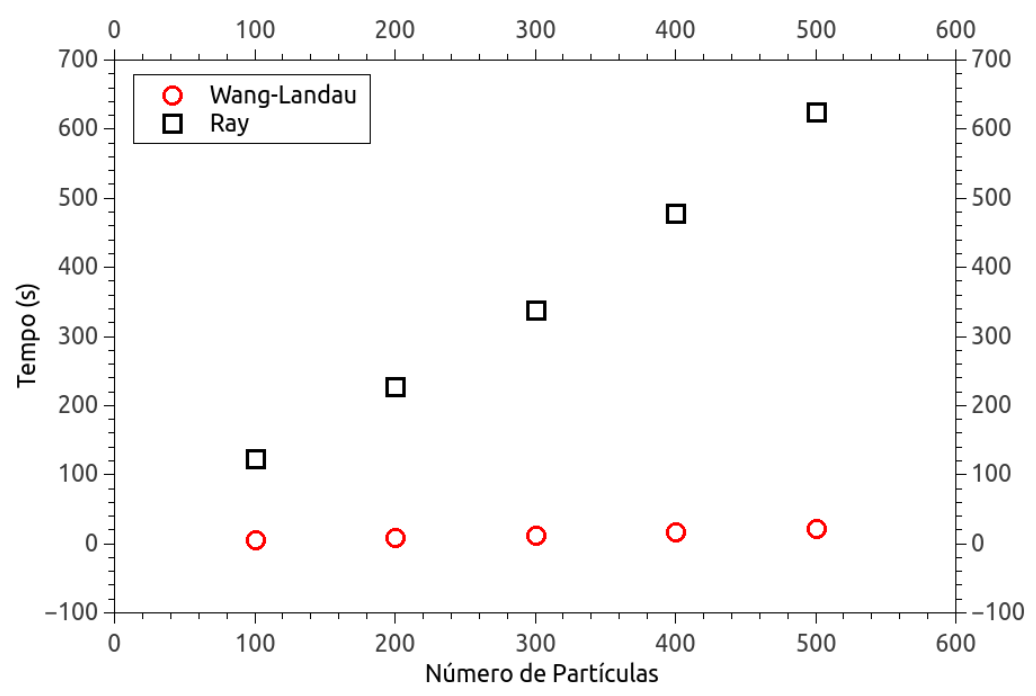

Figura 4.13: Tempos de execução dos métodos de Ray e Wang-Landau para diferentes números de particulas aplicado ao HMF-1D.

A figura 4.13 mostra que o método de Ray é mais sensível a mudanças no número de partículas do que o método de Wang-Landau. Fixando o mesmo número de pontos para ambos os métodos, temos os tempos de execução e o número de passos necessários para que os métodos convirjam aos valores reais. Na tabela 4.3 encontram-se os tempos de execução e número de passos para convergência dos programas, com mesmo número de pontos, para o modelo HMF-1D. 


\begin{tabular}{|c|c|c|c|c|c|c|}
\hline \multirow{2}{*}{$\begin{array}{c}\text { Número de } \\
\text { Partículas }\end{array}$} & \multicolumn{3}{|c|}{ Ray } & \multicolumn{3}{|c|}{ Wang-Landau } \\
\cline { 2 - 7 } & Tempo (s) & $\begin{array}{c}\text { Número } \\
\text { de Passos }\end{array}$ & $\begin{array}{c}\text { Número } \\
\text { de Pontos }\end{array}$ & Tempo (s) & $\begin{array}{c}\text { Número } \\
\text { de Passos }\end{array}$ & $\begin{array}{c}\text { Número } \\
\text { de Pontos }\end{array}$ \\
\hline 100 & 57.53 & $10^{4}$ & 500 & 96.21 & $10^{6}$ & 500 \\
\hline 200 & 449.41 & $2 \times 10^{4}$ & 1000 & 248.58 & $2 \times 10^{6}$ & 1000 \\
\hline 300 & 1518.13 & $3 \times 10^{4}$ & 1500 & 550.87 & $3 \times 10^{6}$ & 1500 \\
\hline 400 & 3917.54 & $4 \times 10^{4}$ & 2000 & 914.17 & $4 \times 10^{6}$ & 2000 \\
\hline 500 & 8224.55 & $5 \times 10^{4}$ & 2500 & 1374.03 & $5 \times 10^{6}$ & 2500 \\
\hline
\end{tabular}

Tabela 4.3: Tempos de execução para diferentes números de partículas dos métodos de Ray e Wang-Landau aplicados ao modelo HMF-1D.

O número de passos necessários em função do número de partículas, com o mesmo número de pontos para ambos os métodos, da tabela 4.3 estão na figura 4.14 .

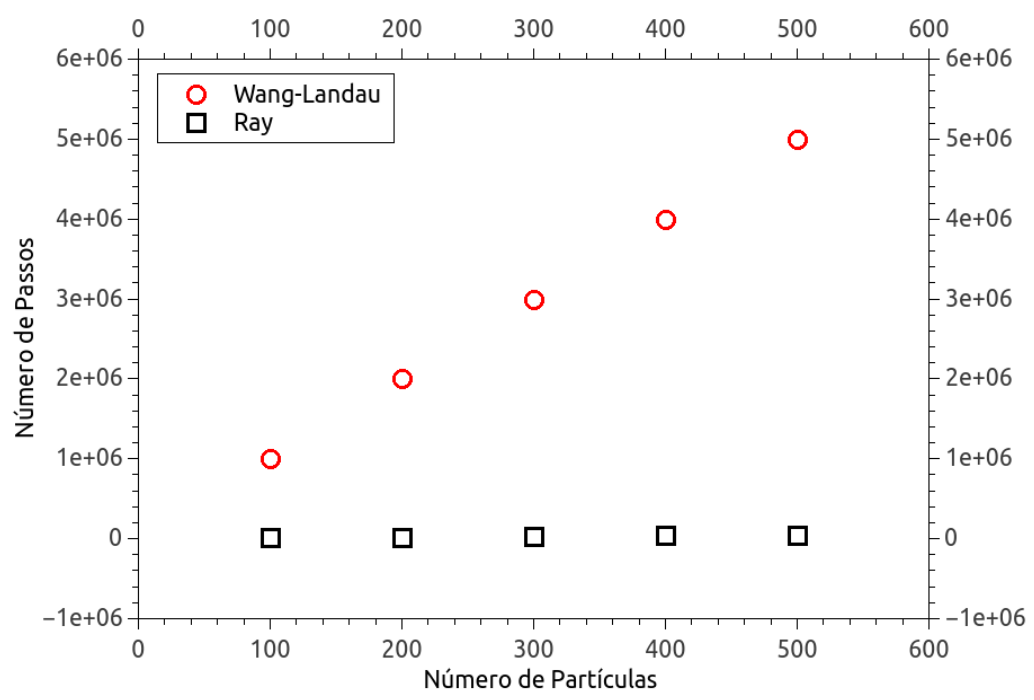

Figura 4.14: Número de passos necessários nos métodos de Ray e Wang-Landau para diferentes números de partículas aplicado ao HMF-1D.

Pela figura 4.14 é possível notar que o método de Wang-Landau necessita de mais passos para convergir quando o número de partículas aumenta. Porém, os números de passos citados aqui são diferentes para cada método. No caso de Ray, o número de passos representa a quantidade de passos que o programa executa para convergir um ponto, enquanto no caso de Wang-Landau, o número de passos representa a quantidade de passos que o programa executa para alterar o fator de modificação $f$. Como o método de Wang-Landau converge todos os pontos de uma vez (ao contrário do método de Ray que converge ponto a ponto), o número de passos para convergir um ponto seria $\frac{N_{\text {pas }} \times N_{\text {mod }}}{N_{\text {pon }}}$, onde $N_{\text {pas }}$ é o número de passos para alterar o fator de modificação, $N_{\text {mod }}$ é o número de vezes que o fator de modificação é alterado (9 vezes, para o método usado neste trabalho) e $N_{\text {pon }}$ é o número de pontos. Com isso, o número de passos para convergir um ponto 
com o método de Wang-Landau no modelo HMF-1D é $1.8 \times 10^{4}$, para qualquer número de partículas da tabela 4.3. Ou seja, o número de passos para convergir um ponto é independente do número de partículas para o método de Wang-Landau, mas cresce com o número de partículas para o método de Ray.

Analisando o tempo de execução de cada programa, levando em conta o número de passos necessários para a convergência e o mesmo número de pontos para ambos os métodos, temos a figura 4.15, usando os dados da tabela 4.3. É possível notar que o método de Wang-Landau apresenta um desempenho melhor do que o método de Ray. Entretanto, o tempo de execução para o método de Ray pode ser diminuido reduzindo o número de pontos calculados. Isso não pode ser feito com o método de Wang-Landau, pois o número de pontos calculados é inversamente proporcional ao tamanho de cada intervalo de energia do histograma. Caso os tamanhos dos intervalos de energia sejam muito grandes, o que corresponde a diminuir o número de pontos calculados, o sistema corre o risco de ficar preso dentro de um desses intervalos de energia e não visitar todas as energias possíveis. Porém, uma forma de reduzir o tempo de execução do método de Wang-Landau, é aplicar o método para vários intervalos de energia separadamente, reduzindo o número de passos necessários para que o sistema visite esses pequenos intervalos, e unir as densidades de estados obtidas em cada intervalo para obter a densidade de estados em todo o intervalo de energia desejado, [25].

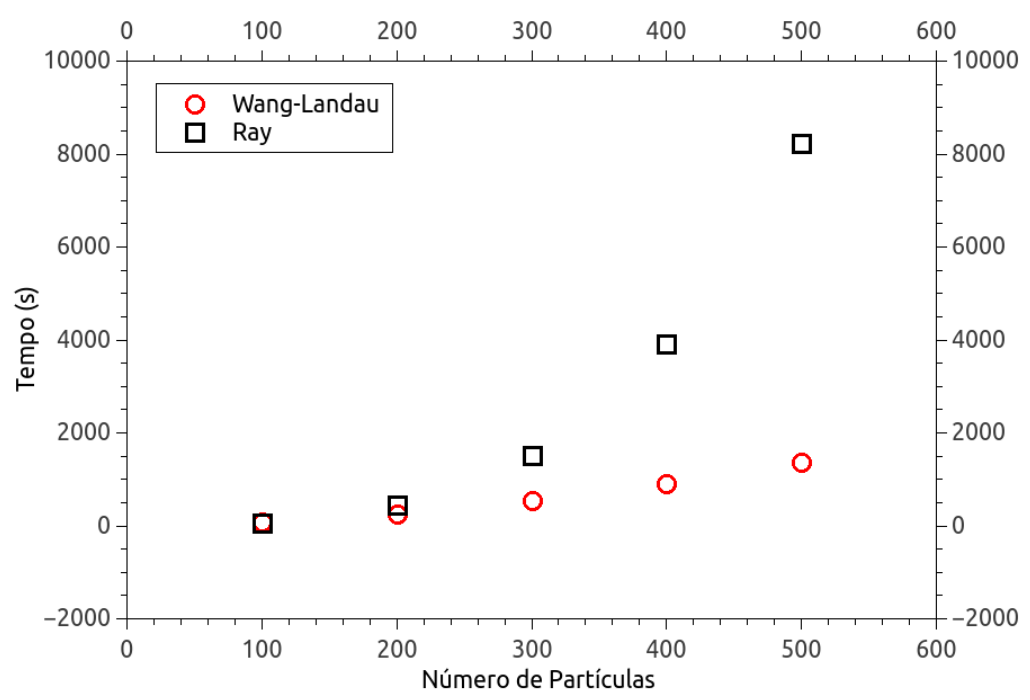

Figura 4.15: Tempos de execução dos métodos de Ray e Wang-Landau aplicado ao HMF$1 \mathrm{D}$. 
Para o cálculo numérico do HMF-2D, ambos os métodos apresentam resultados que estão de acordo com resultados numéricos anteriores, [2, 14, 19, 24], (Figuras 4.10 e 4.12). Assim como no caso do HMF-1D, todas as informações obtidas pelo método de Ray são médias usando a distribuição de posições $\left\{\theta_{i}, \phi_{i}\right\}$. A temperatura é obtida pela equação (3.24), as magnetizações e polarizações pelas equações (4.46)-(4.49) e a entropia, uma vez obtido $T(e)$, pela equação (4.50). Da mesma forma como no HMF-1D, as informações obtidas com o método de Wang-Landau são derivadas da densidade de estados. É importante notar que, pelo método de Wang-Landau, não é possível calcular as magnetizações e polarizações, pois não há uma expressão como (4.42) para elas. Para encontrar expressões deste tipo, seria necessário resolver o modelo HMF-2D analiticamente, assim como foi feito no HMF-1D. Uma forma de encontrar os parâmetros de ordem numericamente pelo método de Wang-Landau, seria encontrar uma entropia que dependa dos parâmetros de ordem e maximizar em relação a eles. Exemplos de como encontrar a entropia como função de mais variáveis são encontrados no trabalho de Shell et al., [22].

Fixando os mesmos números de pontos calculados e números de passos para ambos os métodos, o tempo de execução dos programas para cada algoritmo, no modelo HMF-2D, encontram-se na tabela 4.4 .

\begin{tabular}{|c|c|c|c|c|c|c|}
\hline \multirow{2}{*}{$\begin{array}{c}\text { Número de } \\
\text { Partículas }\end{array}$} & \multicolumn{3}{|c|}{ Ray } & \multicolumn{3}{|c|}{ Wang-Landau } \\
\cline { 2 - 7 } & Tempo (s) & $\begin{array}{c}\text { Número } \\
\text { de Passos }\end{array}$ & $\begin{array}{c}\text { Número } \\
\text { de Pontos }\end{array}$ & Tempo (s) & $\begin{array}{c}\text { Número } \\
\text { de Passos }\end{array}$ & $\begin{array}{c}\text { Número } \\
\text { de Pontos }\end{array}$ \\
\hline 100 & 47.34 & $10^{4}$ & $10^{2}$ & 1.88 & $10^{4}$ & $10^{2}$ \\
\hline 200 & 107.33 & $10^{4}$ & $10^{2}$ & 3.82 & $10^{4}$ & $10^{2}$ \\
\hline 300 & 179.38 & $10^{4}$ & $10^{2}$ & 6.40 & $10^{4}$ & $10^{2}$ \\
\hline 400 & 247.83 & $10^{4}$ & $10^{2}$ & 9.33 & $10^{4}$ & $10^{2}$ \\
\hline 500 & 316.55 & $10^{4}$ & $10^{2}$ & 12.05 & $10^{4}$ & $10^{2}$ \\
\hline
\end{tabular}

Tabela 4.4: Tempos de execução com número de passos e pontos fixados para diferentes números de partículas dos métodos de Ray e Wang-Landau aplicados ao modelo HMF-2D.

O tempo de execução em função do número de partículas, para número de passos e pontos fixados, da tabela 4.4 estão na figura 4.16. A figura 4.16 mostra que o método de Ray é mais sensível a mudanças no número de partículas, assim como é observado no HMF-1D (Figura 4.13). 


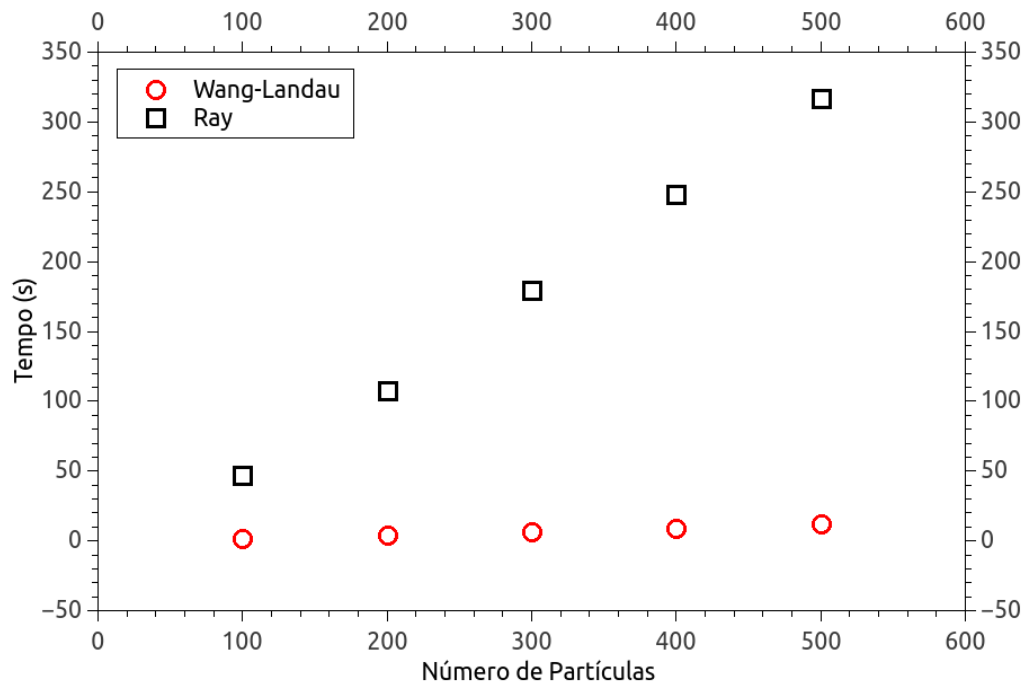

Figura 4.16: Tempos de execução entre dos métodos de Ray e Wang-Landau para diferentes números de partículas aplicado ao HMF-2D.

Escolhendo o mesmo número de pontos calculados em ambos os métodos, temos os tempos de execução e o número de passos necessários para que os métodos convirjam aos valores reais. A tabela 4.5 mostra os tempos de execução e número de passos para convergência dos programas, com mesmo número de pontos calculados, para o modelo HMF-2D.

\begin{tabular}{|c|c|c|c|c|c|c|}
\hline \multirow{2}{*}{$\begin{array}{c}\text { Número de } \\
\text { Partículas }\end{array}$} & \multicolumn{3}{|c|}{ Ray } & \multicolumn{3}{|c|}{ Wang-Landau } \\
\cline { 2 - 7 } & Tempo (s) & $\begin{array}{c}\text { Número } \\
\text { de Passos }\end{array}$ & $\begin{array}{c}\text { Número } \\
\text { de Pontos }\end{array}$ & Tempo (s) & $\begin{array}{c}\text { Número } \\
\text { de Passos }\end{array}$ & $\begin{array}{c}\text { Número } \\
\text { de Pontos }\end{array}$ \\
\hline 20 & 86.58 & $2 \times 10^{4}$ & 300 & 155.78 & $2 \times 10^{6}$ & 300 \\
\hline 40 & 479.20 & $4 \times 10^{4}$ & 600 & 468.49 & $4 \times 10^{6}$ & 600 \\
\hline 60 & 1479.52 & $6 \times 10^{4}$ & 900 & 938.18 & $6 \times 10^{6}$ & 900 \\
\hline 80 & 3395.63 & $8 \times 10^{4}$ & 1200 & 1598.79 & $8 \times 10^{6}$ & 1200 \\
\hline 100 & 6628.30 & $10^{5}$ & 1500 & 2521.32 & $10^{7}$ & 1500 \\
\hline
\end{tabular}

Tabela 4.5: Tempos de execução para diferentes números de partículas dos métodos de Ray e Wang-Landau aplicados ao modelo HMF-2D.

Os valores dos números de passos necessários em função do número de partículas, com o mesmo número de pontos para ambos os métodos, da tabela 4.5 estão na figura 4.17. Aparentemente o método de Wang-Landau necessita de mais passos quando o número de partículas aumenta (Figura 4.17), mas o mesmo argumento usado no HMF-1D é aplicado aqui, de tal forma que o número de passos para convergir um ponto com o método de Wang-Landau, para o HMF-2D, é $6 \times 10^{4}$, para qualquer número de partículas da tabela 4.5. Assim como no HMF-1D, o número de passos para convergir um ponto no método de Wang-Landau é independente do número de partículas, mas aumenta com o número de partículas no método de Ray. 


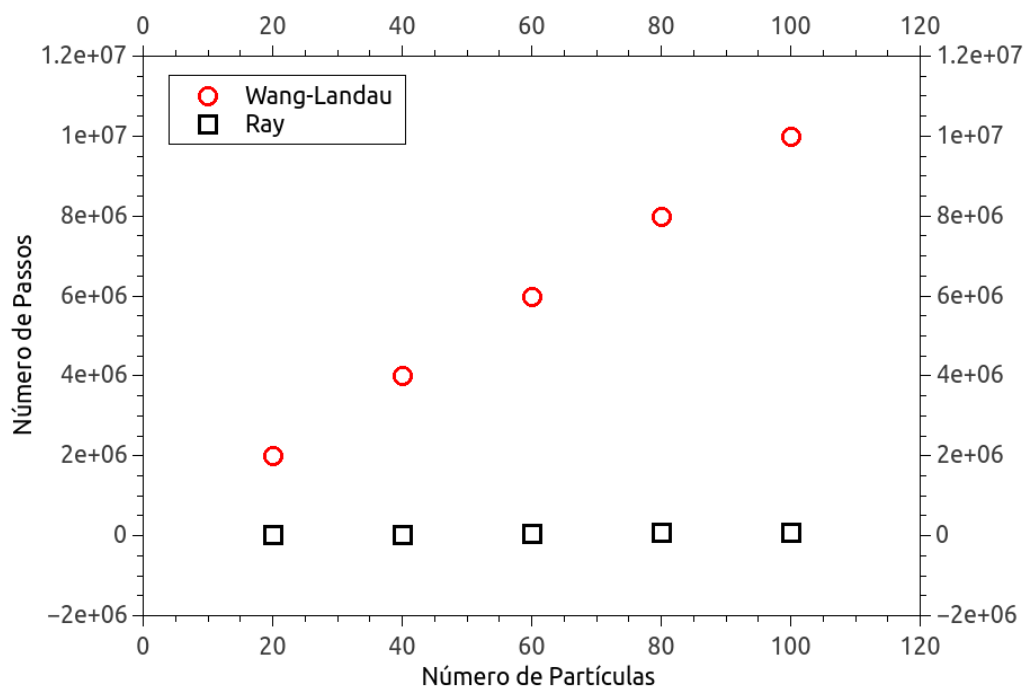

Figura 4.17: Número de passos necessários nos métodos de Ray e Wang-Landau para diferentes números de partículas aplicado ao HMF-2D.

Olhando para o tempo de execução de cada programa, levando em consideração o número de passos necessários para a convergência e o mesmo número de pontos para ambos os métodos, temos a figura 4.18, usando os dados da tabela 4.5. Assim como no modelo HMF-1D, o método de Wang-Landau apresenta um desempenho superior ao método de Ray. Mas, deve-se levar em consideração que ambos os métodos possuem formas de terem seus tempos de execução reduzidos. Sendo elas: a redução no número de pontos calculados, para o método de Ray, e o cálculo em diferentes intervalos de energia, para o método de Wang-Landau.

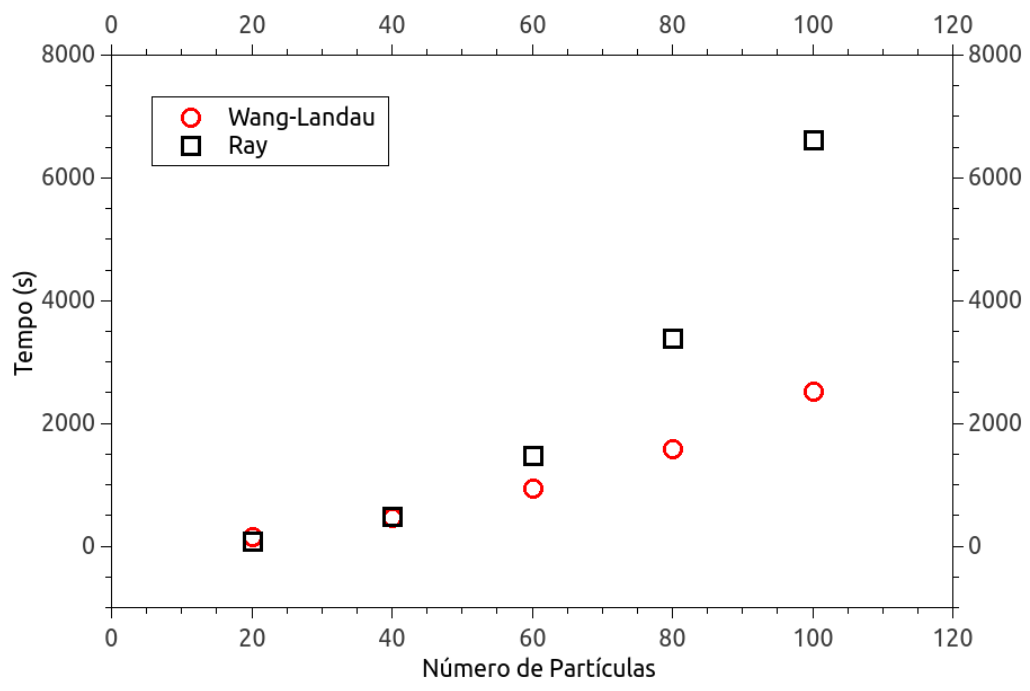

Figura 4.18: Tempos de execução dos métodos de Ray e Wang-Landau aplicado ao HMF2D. 


\section{Conclusão}

Como foi mostrado no Capítulo 2, sistemas com interações de longo alcance possuem energia não-aditiva e podem apresentar inequivalência entre os ensembles. Como visto no Capítulo 1, a construção do ensemble canônico supõe a aditividade da energia, ou seja, para estudar sistemas com interações de longo alcance é recomendado o ensemble microcanônico. Neste trabalho foi apresentada uma análise de dois métodos de Monte Carlo microcanônico, para estudar a termodinâmica de sistemas com interações de longo alcance.

A proposta apresentada nesse trabalho de um novo critério para alterar o fator de modificação $f$, no método de Wang-Landau, mostrou-se tão eficiente quanto as propostas anteriores, [22, 25], com a vantagem de reduzir a variação do tempo de cálculo computacional do método. No que diz respeito a aplicabilidade dos métodos, o de Wang-Landau pode ser aplicado a sistemas discretos, o que não é possível com o método de Ray. O método de Wang-Landau é recomendado quando buscamos informações do sistema que são derivadas de um potencial termodinâmico, como a entropia. Enquanto que o de Ray é recomendado quando as grandezas a serem calculadas são obtidas através de médias usando a distribuição de posições do sistema. Considerando o desempenho dos métodos, o de Wang-Landau apresenta melhores resultados, quando a quantidade de informação e precisão exigida é a mesma para os dois. 
float $* \mathrm{X}=($ float $*)$ malloc $(\mathrm{N} *$ sizeof $($ float $))$;

float $* X 2=($ float $*)$ malloc $(N * \operatorname{sizeof}($ float $))$;

//INICIA A PRIMEIRA SEQUÊNCIA

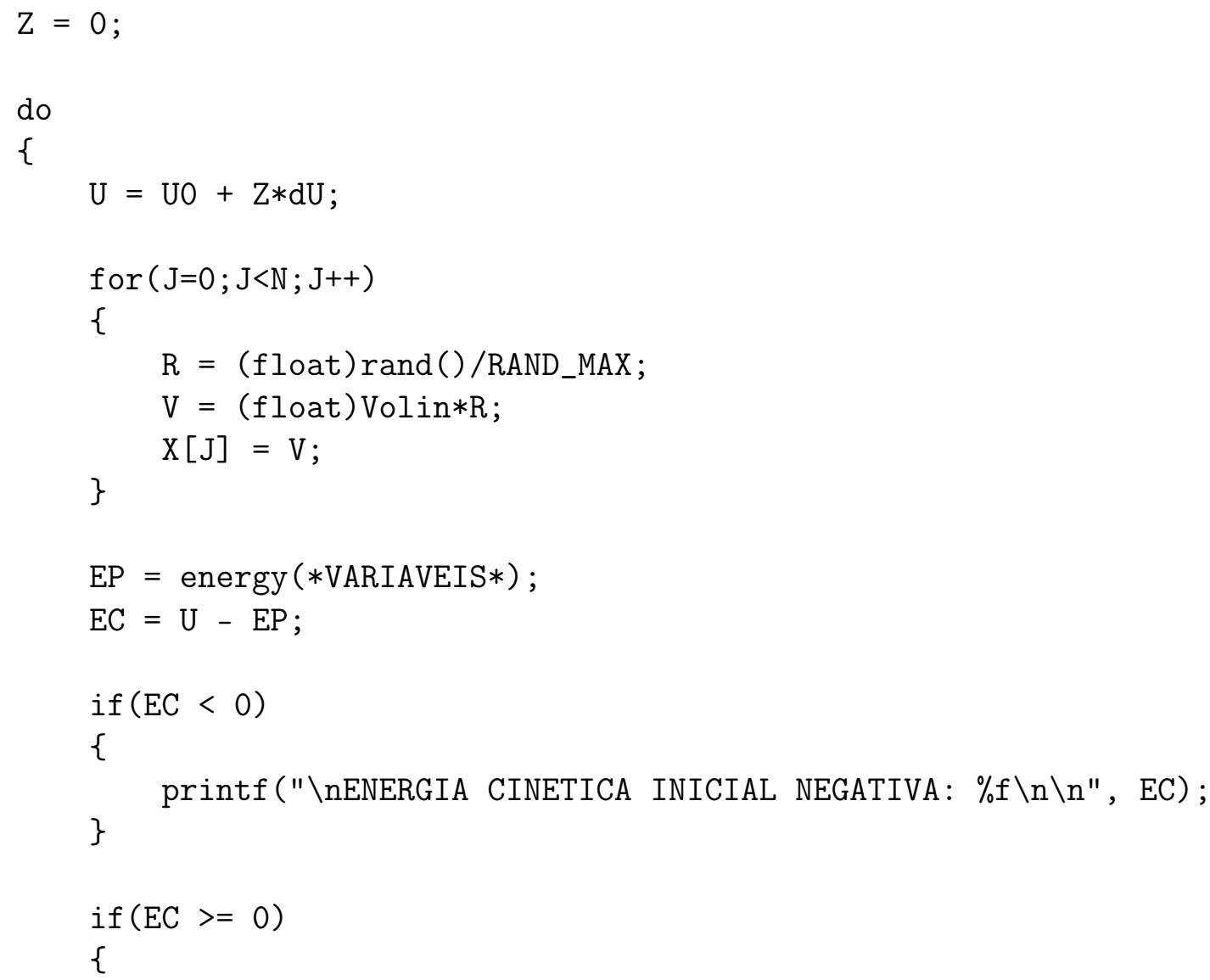

//COMEÇA O LOOP

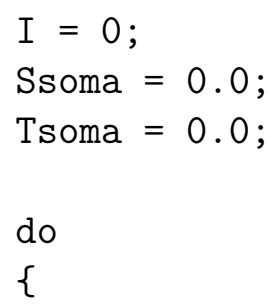

//PROPÕE UM NOVO ESTADO

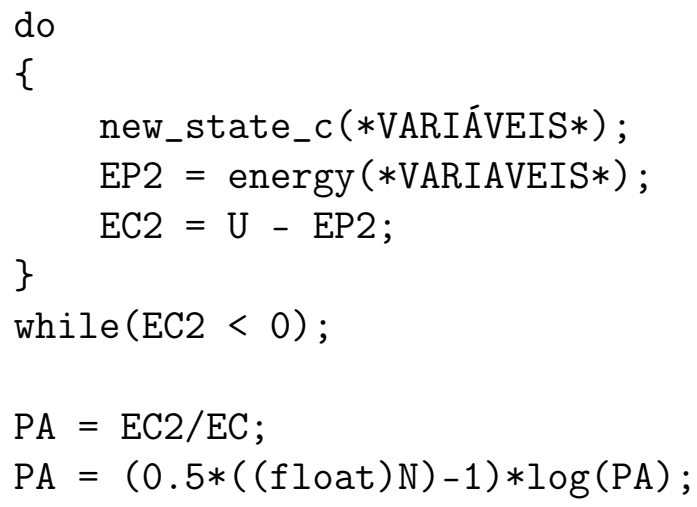




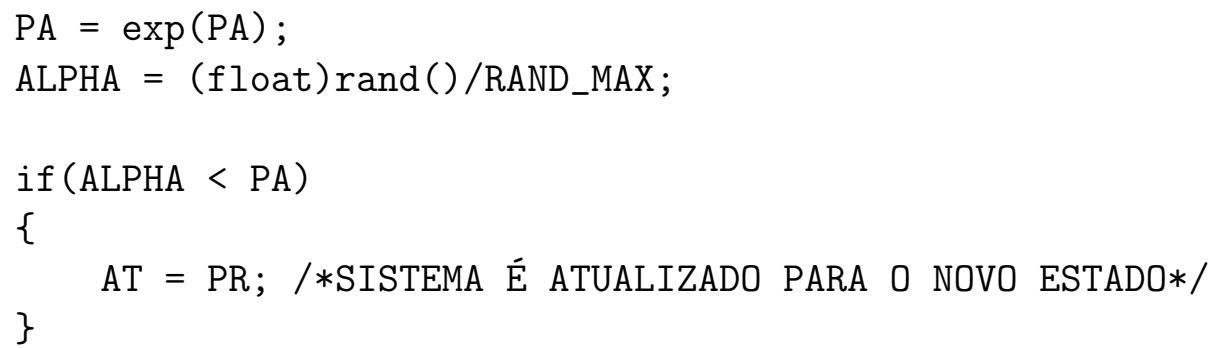

//COMPUTA A ENTROPIA E TEMPERATURA

$$
\begin{aligned}
& \mathrm{S}=\text { entropy }(* \operatorname{VARIÁVEIS~} *) ; \\
& \mathrm{T}=\text { temperature }(* \operatorname{VARIÁVEIS~} *)
\end{aligned}
$$

//REALIZA A MEDIA DOS VALORES

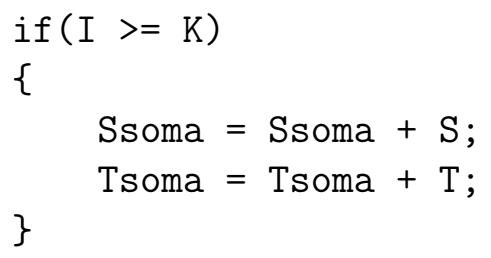

//INCREMENTA O CONTATOR DE PASSOS

$$
I=I+1
$$

//TESTE PARA FINALIZACAO

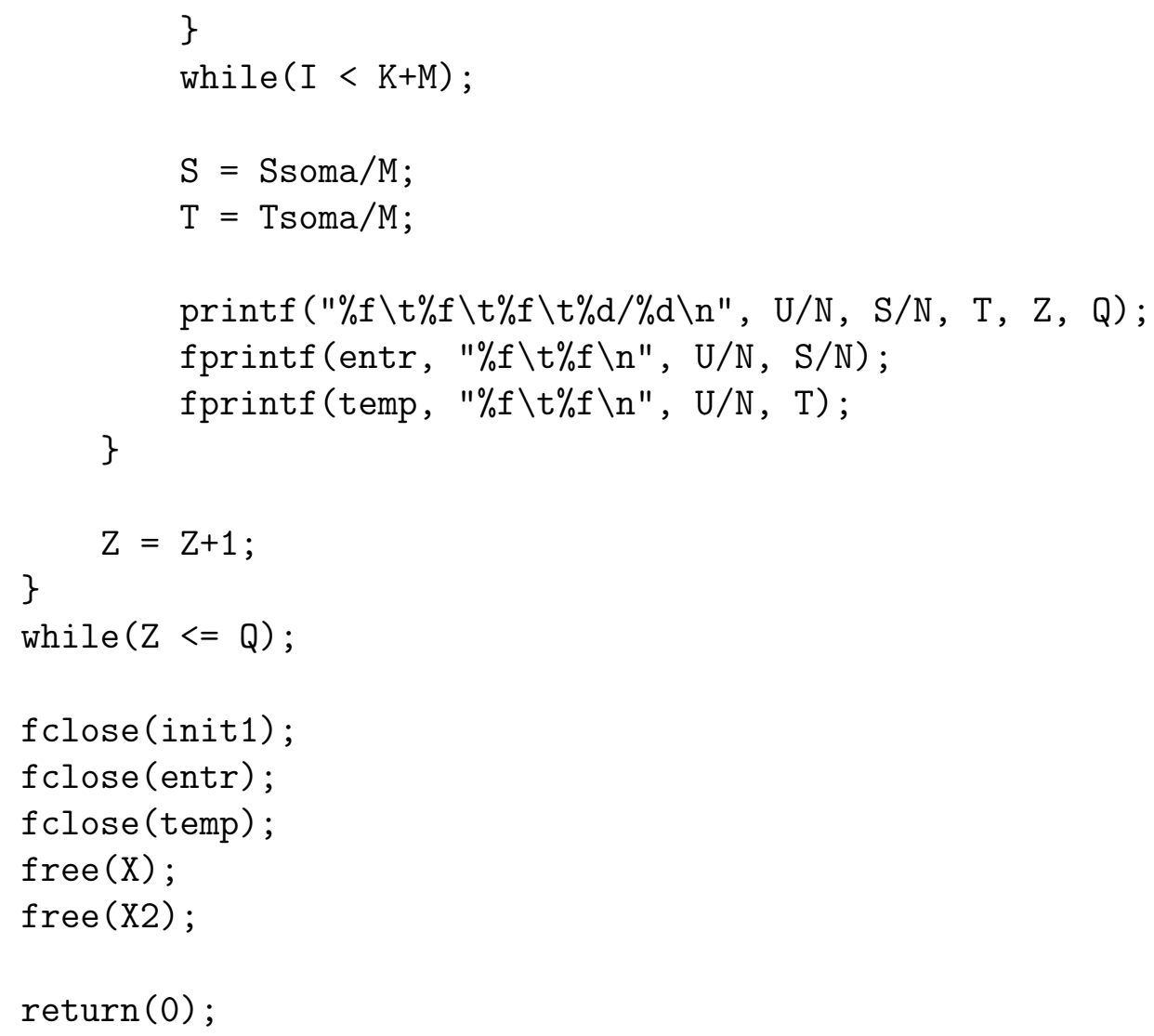




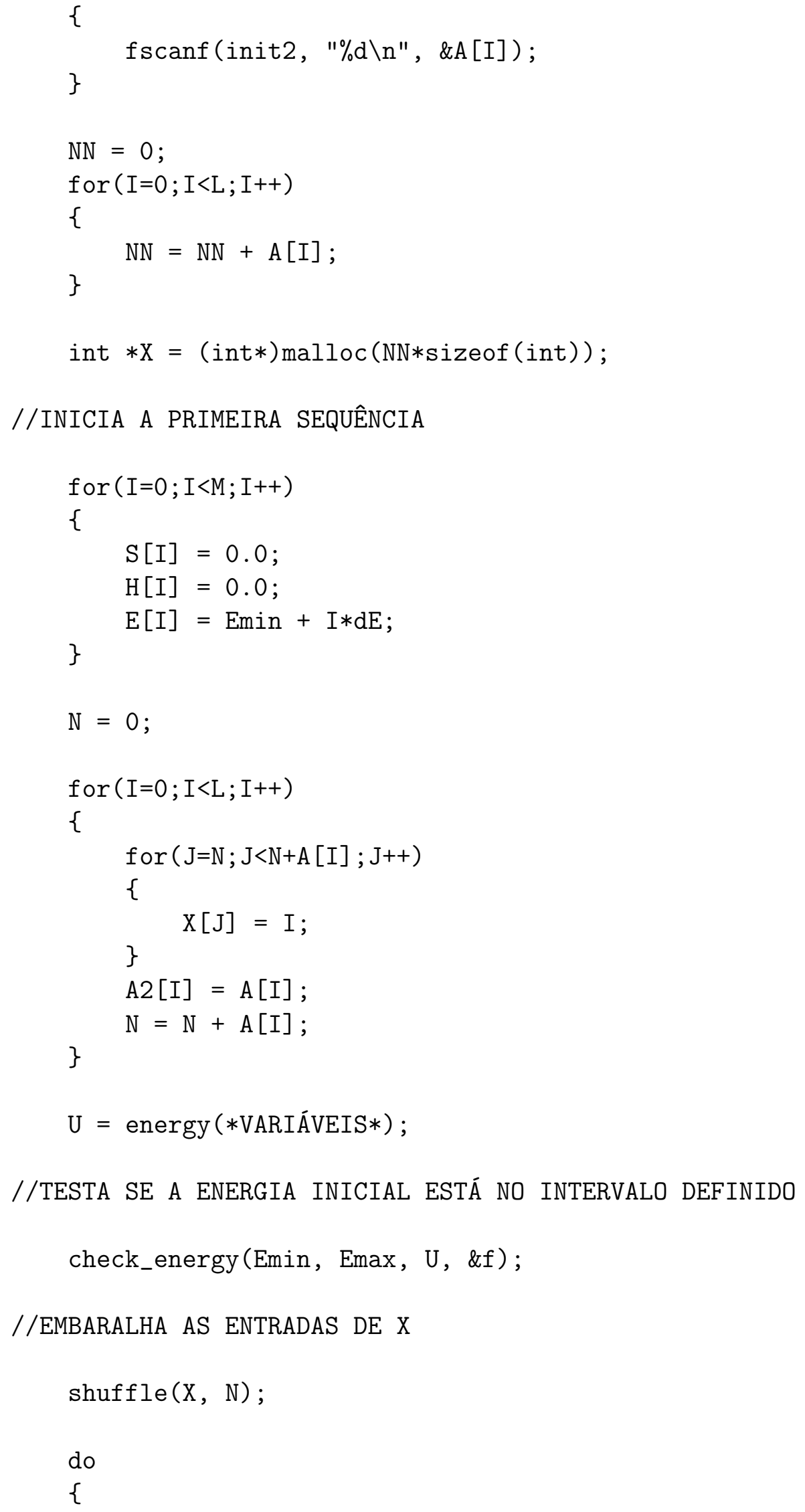

//TESTA SE A ENERGIA INICIAL ESTÁ NO INTERVALO DEFINIDO check_energy(Emin, Emax, U, \&f);

//EMBARALHA AS ENTRADAS DE X

$\operatorname{shuffle}(X, N)$;

do

\{

//PROPÕE UM NOVO ESTADO 


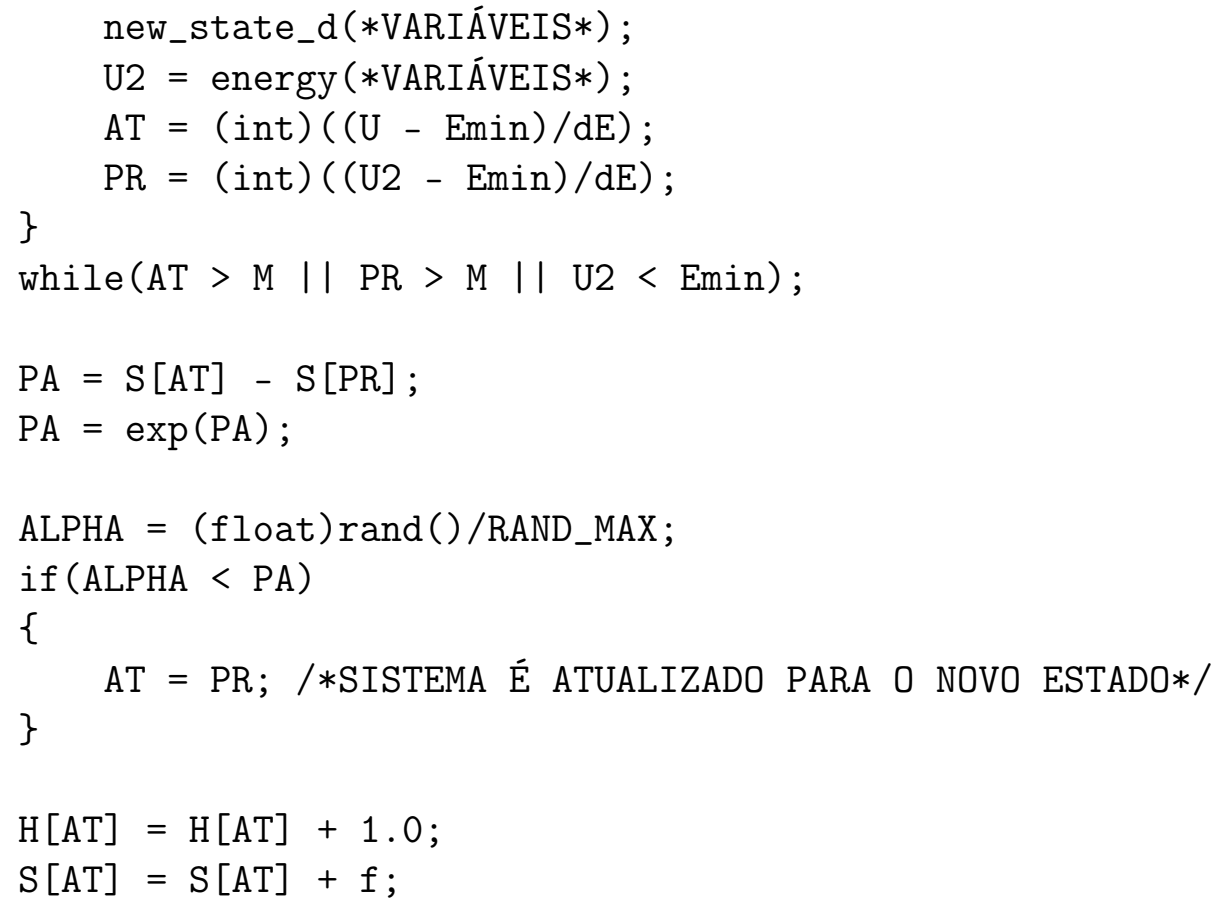

//APLICA O CRITÉRIO PARA DIMINUIR O FATOR DE MODIFICAÇÃO

check_flat (M, \&t, $H, f l a t, \& f)$;

check_minimum (M, \&t, H, minimo, \&f $) ; / *$ ESCOLHER APENAS UM $* /$ check_step (M, \&t, H, minimo, \&f);

//REESCALA A ENTROPIA

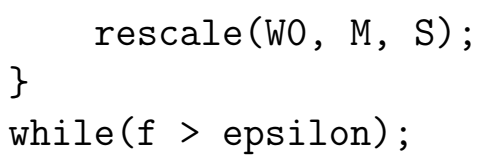

//COMPUTA A ENTROPIA

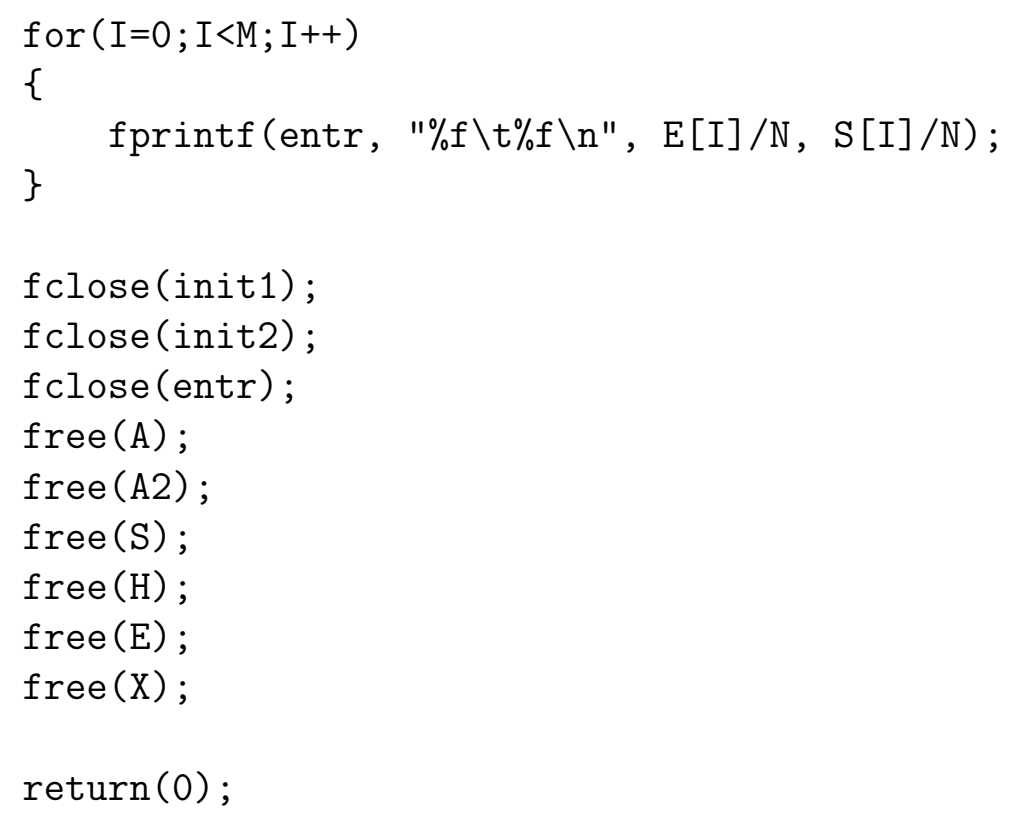


//INICIA A PRIMEIRA SEQUÊNCIA

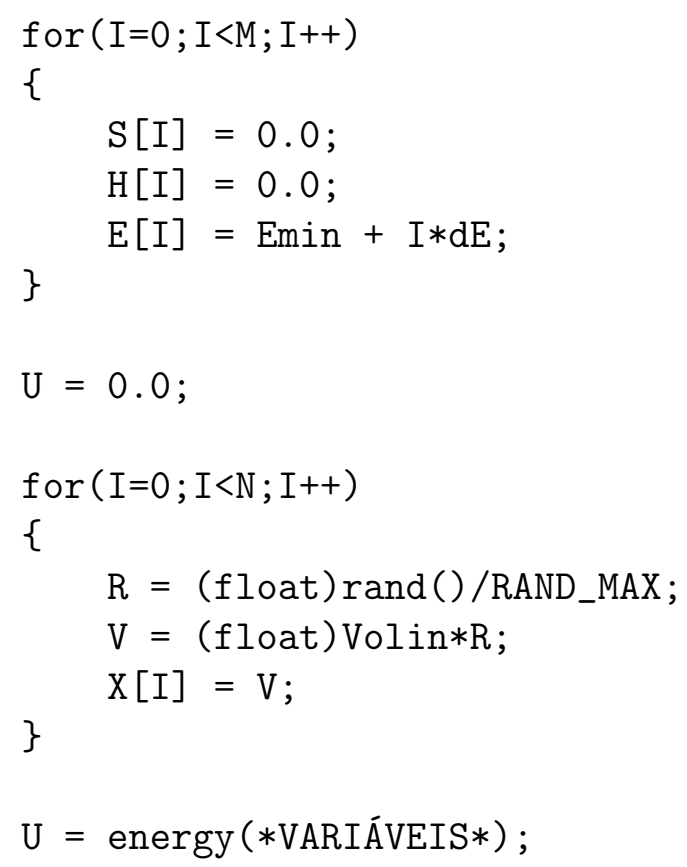

//TESTA SE A ENERGIA ESTÁ NO INTERVALO DEFINIDO

check_energy(Emin, Emax, U, \&f);

do

\{

//PROPÕE UM NOVO ESTADO

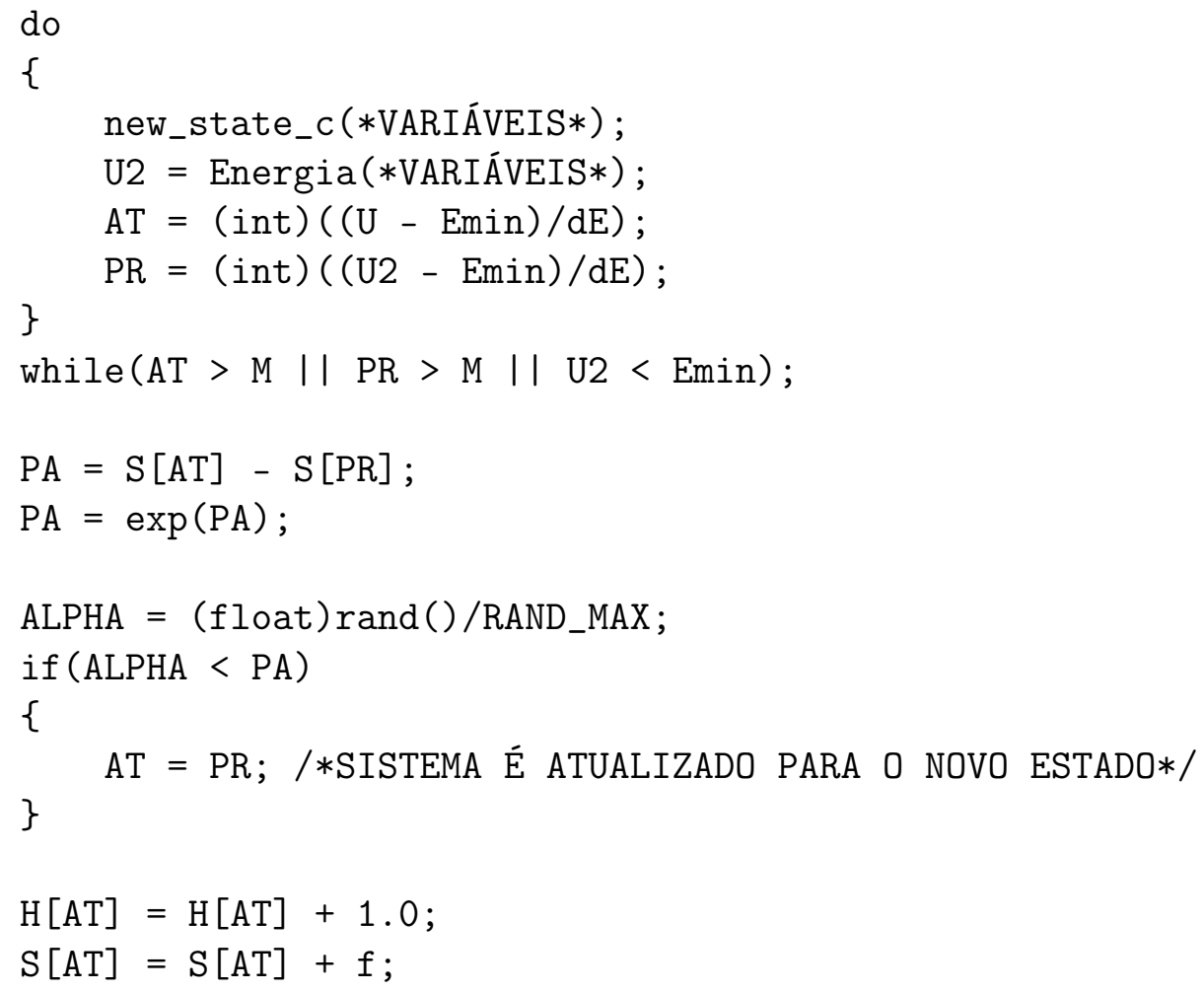


//APLICA O CRITÉRIO PARA DIMINUIR O FATOR DE MODIFICAÇÃO

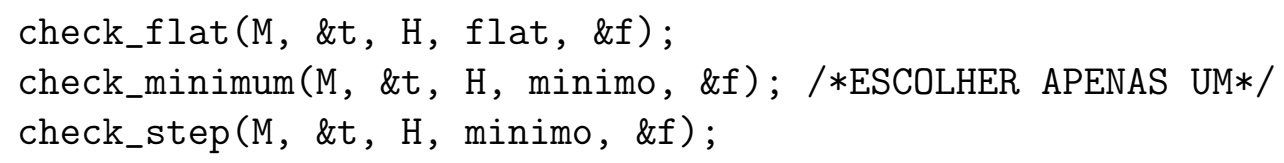

//REESCALA A ENTROPIA

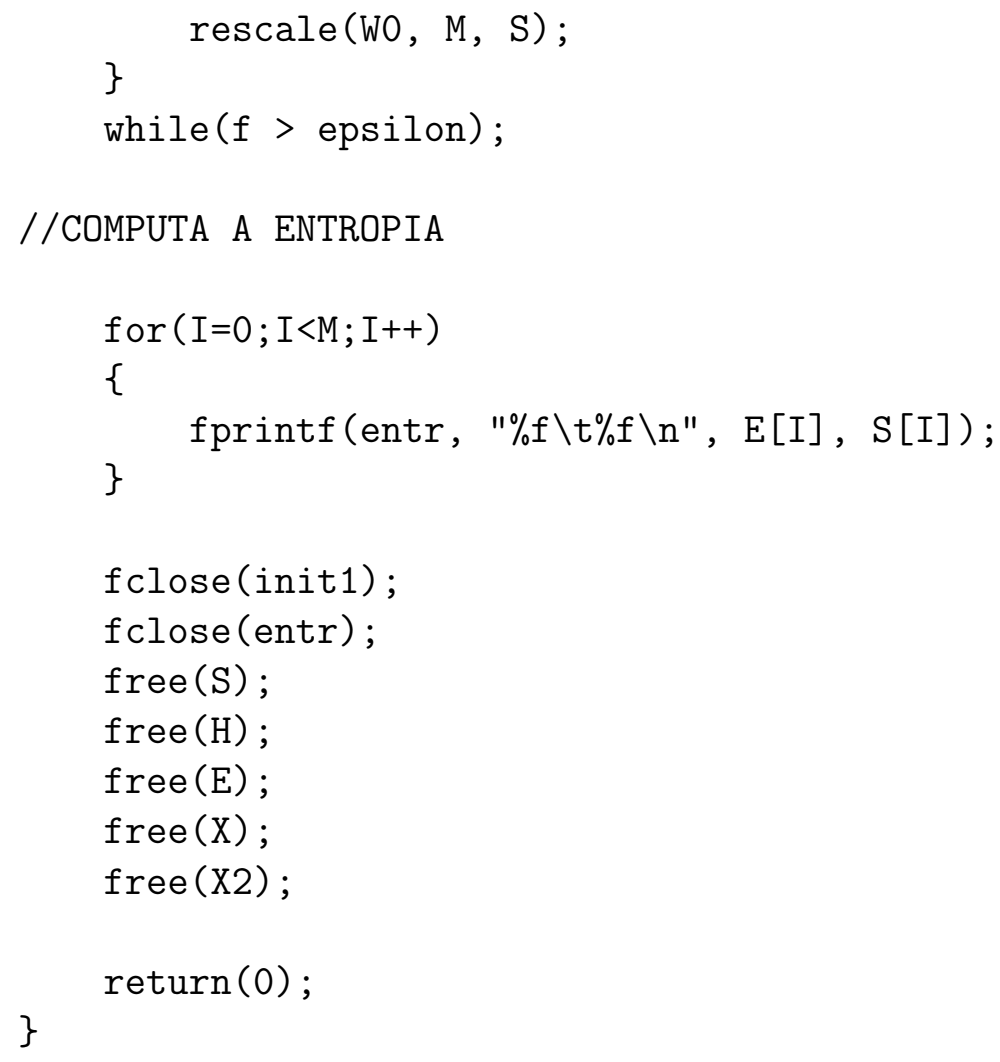


- Modificador de Estados Discreto

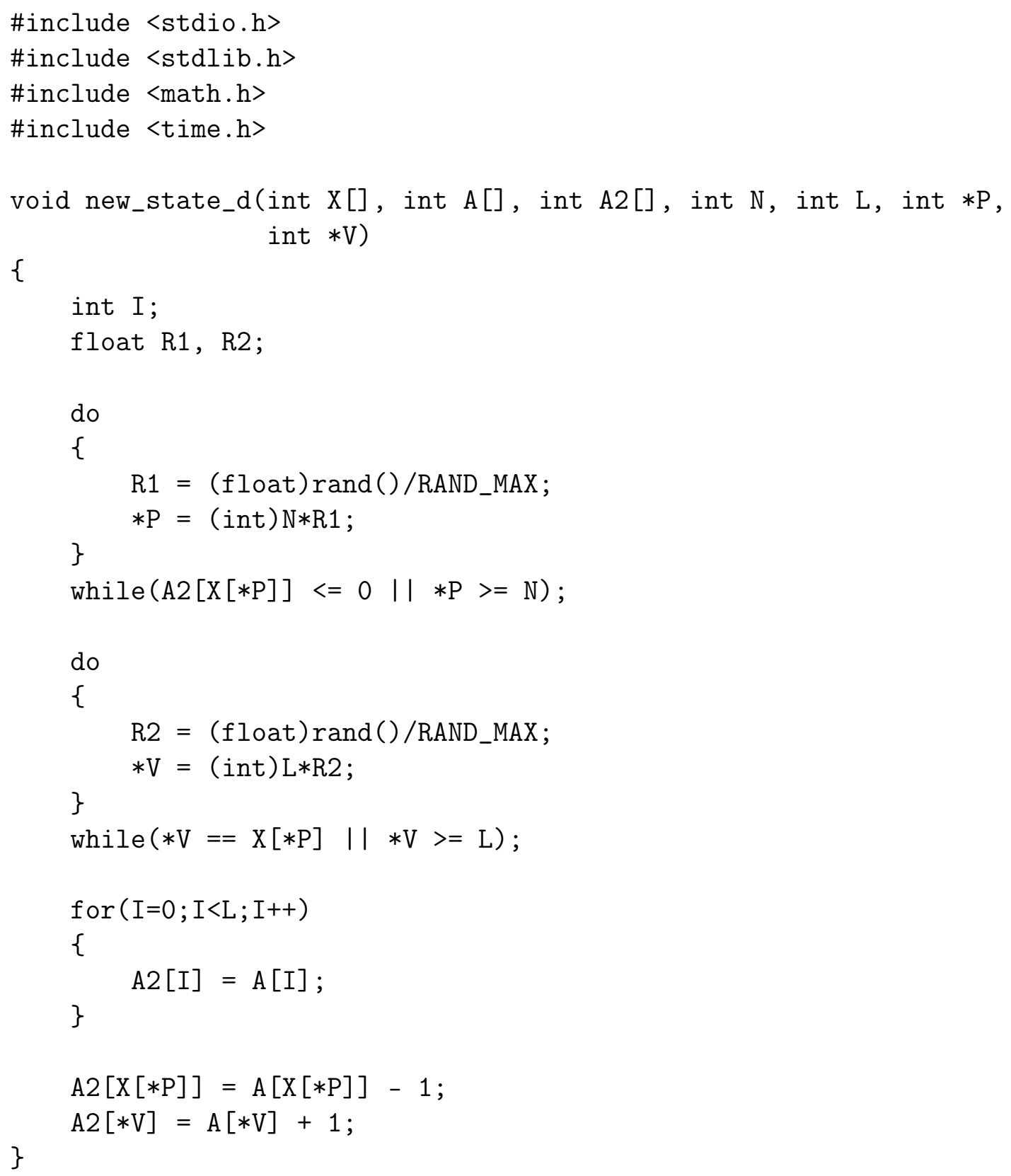


- Modificador de Estados Contínuo

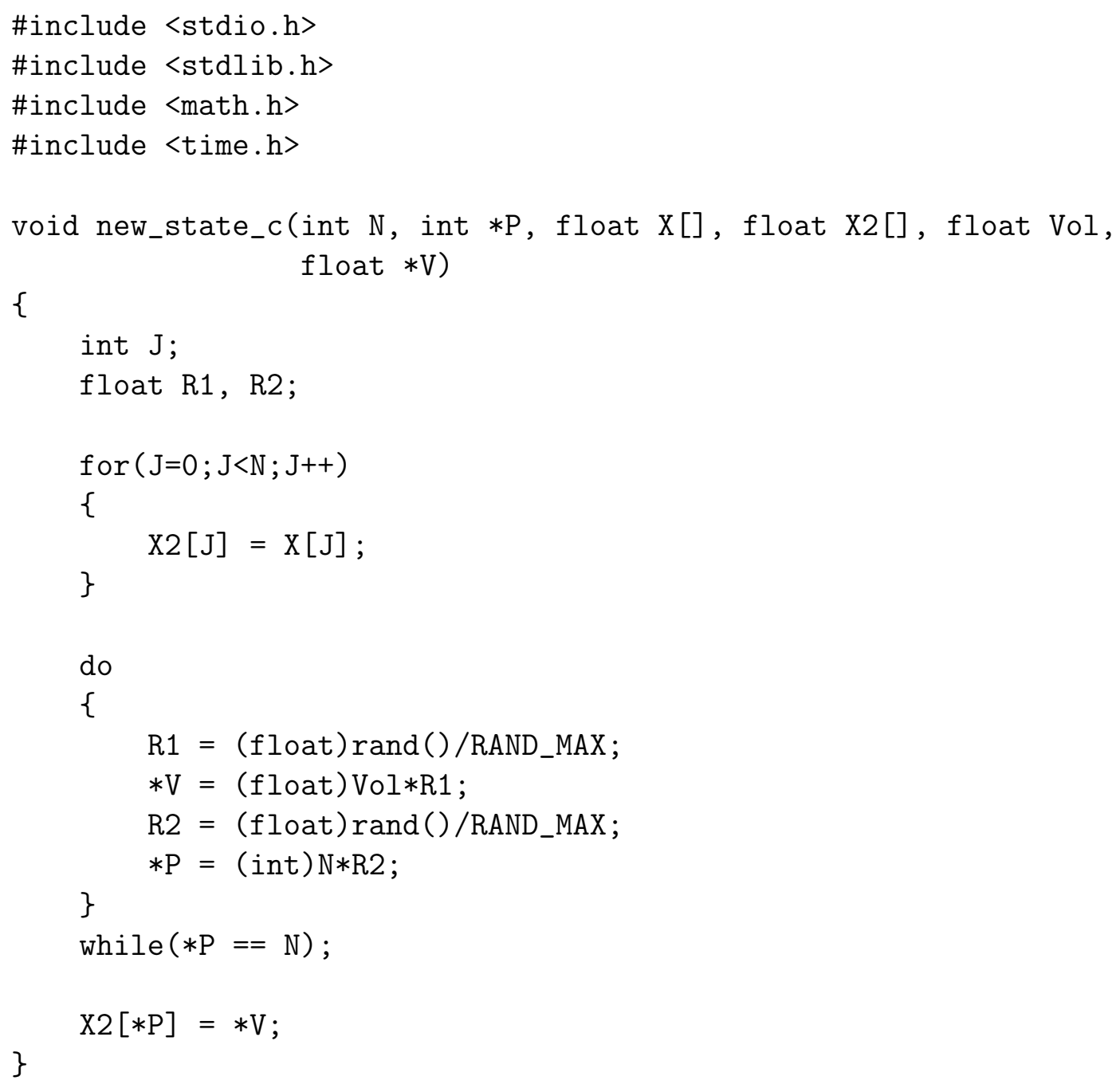




\section{Referências Bibliográficas}

[1] Mickael Antoni and Stefano Ruffo. Clustering and relaxation in hamiltonian longrange dynamics. Physical Review E, 52(3):2361, 1995.

[2] Mickael Antoni, Stefano Ruffo, and Alessandro Torcini. First-and second-order clustering transitions for a system with infinite-range attractive interaction. Physical Review E, 66(2):025103, 2002.

[3] Mickaël Antoni and Alessandro Torcini. Anomalous diffusion as a signature of a collapsing phase in two-dimensional self-gravitating systems. Physical Review E, 57(6):R6233, 1998.

[4] RE Belardinelli and VD Pereyra. Wang-landau algorithm: A theoretical analysis of the saturation of the error. The Journal of chemical physics, 127(18):184105, 2007.

[5] Herbert B Callen. Thermodynamics and an Introduction to Thermostatistics. John wiley \& sons, 1985.

[6] Alessandro Campa, Thierry Dauxois, and Stefano Ruffo. Statistical mechanics and dynamics of solvable models with long-range interactions. Physics Reports, 480(3):57$159,2009$.

[7] Alvaro de Almeida Caparica and AG Cunha-Netto. Wang-landau sampling: Improving accuracy. Physical Review E, 85(4):046702, 2012.

[8] Michael Creutz. Microcanonical monte carlo simulation. Physical Review Letters, 50(19):1411, 1983.

[9] Markus Deserno. Microcanonical and canonical two-dimensional ising model: An example. UCLA, USA, 2004.

[10] M. A. Amato e L. S. F Olavo. Introdução à Física. Editora Universidade de Brasília, 2013. 
[11] Dieter HE Gross. Microcanonical Thermodynamics: Phase transitions in "small"systems, volume 66. World Scientific, 2001.

[12] Edwin T Jaynes. Information theory and statistical mechanics. Physical review, 106(4):620, 1957.

[13] Helmut G Katzgraber. Introduction to monte carlo methods. arXiv preprint arXiv:0905.1629, 2009.

[14] JM Maciel, M-C Firpo, and MA Amato. Some statistical equilibrium mechanics and stability properties of a class of two-dimensional hamiltonian mean-field models. Physica A: Statistical Mechanics and its Applications, 424:34-43, 2015.

[15] Nicholas Metropolis, Arianna W Rosenbluth, Marshall N Rosenbluth, Augusta H Teller, and Edward Teller. Equation of state calculations by fast computing machines. The journal of chemical physics, 21(6):1087-1092, 1953.

[16] RK Pathria. Statistical mechanics. 1996.

[17] Eric M Pearson, Timur Halicioglu, and William A Tiller. Laplace-transform technique for deriving thermodynamic equations from the classical microcanonical ensemble. Physical Review A, 32(5):3030, 1985.

[18] John R Ray. Microcanonical ensemble monte carlo method. Physical Review A, 44(6):4061, 1991.

[19] TM Rocha Filho, MA Amato, and A Figueiredo. A novel approach to the determination of equilibrium properties of classical hamiltonian systems with long-range interactions. Journal of Physics A: Mathematical and Theoretical, 42(16):165001, 2009 .

[20] Sílvio Roberto Salinas. Introdução a Física Estatística Vol. 09. Edusp, 1997.

[21] Claude E Shannon and W Weaver. The mathematical theory of communication. University of Illinois Press, 1949.

[22] M Scott Shell, Pablo G Debenedetti, and Athanassios Z Panagiotopoulos. Generalization of the wang-landau method for off-lattice simulations. Physical review E, 66(5):056703, 2002. 
[23] H Eugene Stanley. Introduction to phase transitions and critical phenomena. Oxford University Press, 1987.

[24] Alessandro Torcini and Mickaël Antoni. Equilibrium and dynamical properties of two-dimensional n-body systems with long-range attractive interactions. Physical Review E, 59(3):2746, 1999.

[25] Fugao Wang and DP Landau. Efficient, multiple-range random walk algorithm to calculate the density of states. Physical review letters, 86(10):2050, 2001.

[26] Julia M Yeomans. Statistical mechanics of phase transitions. Oxford University Press, 1992. 